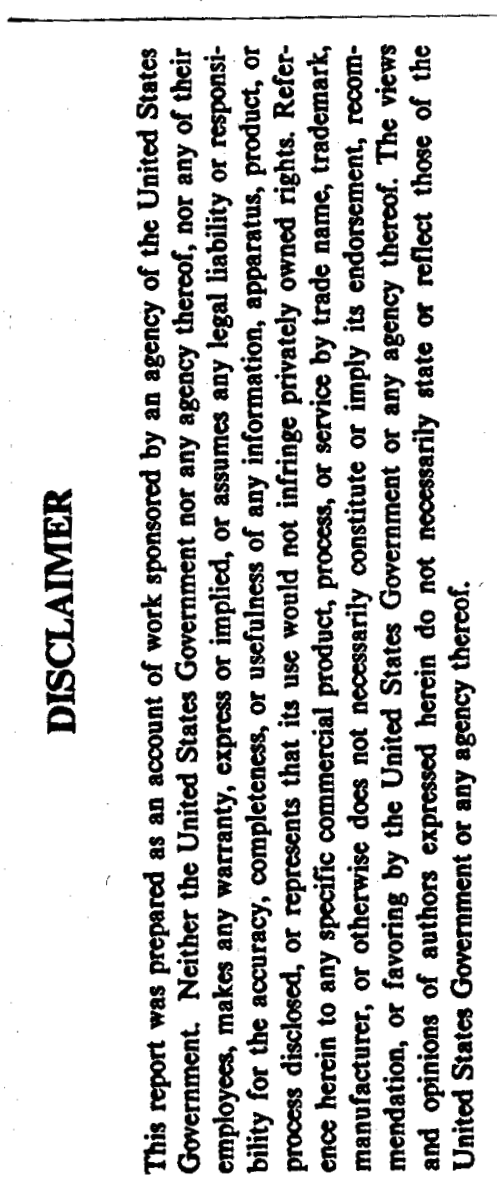

SGP-TR-120

\title{
Stanford Geothermal Program
}

Fourth Annual Report

Reservoir and Injection

Technology

\author{
Principal Investigators: \\ Roland N. Horne, H.J. Ramèy, Jr., \\ F.G. Miller, W.E. Brigham, \\ Paul Kruger
}

December, 1988

Department of Energy Contract Number DE-AS07-84ID12529

For the Period

October 1, 1987 through December 31, 1988

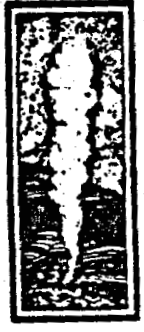

Stanford Geothermal Program Interdisciplinary Research in Engineering and Earth Sciences STANFORD UNIVERSITY Stanford, California 


\section{DISCLAIMER}

This report was prepared as an account of work sponsored by an agency of the United States Government. Neither the United States Government nor any agency Thereof, nor any of their employees, makes any warranty, express or implied, or assumes any legal liability or responsibility for the accuracy, completeness, or usefulness of any information, apparatus, product, or process disclosed, or represents that its use would not infringe privately owned rights. Reference herein to any specific commercial product, process, or service by trade name, trademark, manufacturer, or otherwise does not necessarily constitute or imply its endorsement, recommendation, or favoring by the United States Government or any agency thereof. The views and opinions of authors expressed herein do not necessarily state or reflect those of the United States Government or any agency thereof. 


\section{DISCLAIMER}

Portions of this document may be illegible in electronic image products. Images are produced from the best available original document. 


\section{Contents}

1 Application of Boundary Element Method to Front Tracking and Pressure Transient Testing 6

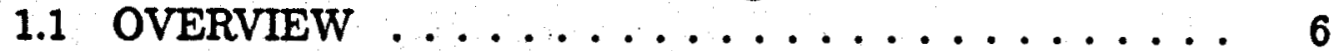

1.2 INTRODUCTION ................. 7

1.3 THEORETICAL CONSIDERATIONS . . . . . . . . 10

1.4 Boundary Behavior . . . . . . . . . . . 14

1.5 STEADY STATE PROBLEMS . . . . . . . . . 15

1.6 UNSTEADY STATE PROBLEMS . . . . . . . . . 21

1.7 LIMITATIONS $\ldots \ldots \ldots \ldots \ldots \ldots \ldots \ldots \ldots$

1.8 CONCLUSIONS ......................... 31

1.9 NOMENCLATURE . . . . . . . . . . . . . 32

1.10 REFERENCES . . . . . . . . . . . . . . 33

2 Determination of Fracture Aperture - A Multi-Tracer Approach $\quad 36$

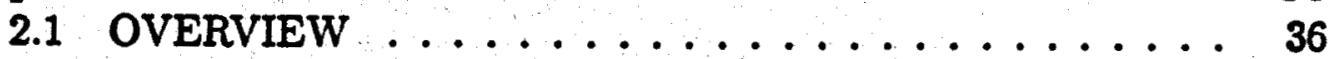

2.2 INTRODUCTION ....................... 37

2.3 THEORY ............................ 37

2.4 ANALYSIS OF WAIRAKEI FIELD DATA ...... 50

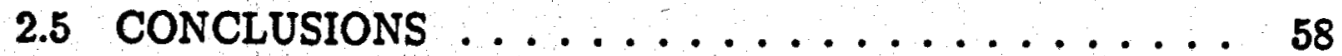

2.6 NOMENCLATURE .................... 60

2.7 REFERENCES ..................... 61

3 Analysis of Tracer and Thermal Transients During Reinjection

3.1 INTRODUCTION ...................... 63

3.2 INTERWELL TESTS - NO RECIRCULATION . . . . 66 
3.3 INTERWELL TESTS WITH RECIRCULATION . . . . 75 3.4 CONCLUSIONS ............... 77

4 Pressure Transient Modeling of Non-Uniformly Fractures Reservoirs 82 4.1 OVERVIEW $\ldots \ldots \ldots \ldots \ldots \ldots \ldots \ldots \ldots$

4.2 INTRODUCTION ................... 82

4.3 THEORY ..................... 83

4.4 SOLUTION . . . . . . . . . . . . . . . 84

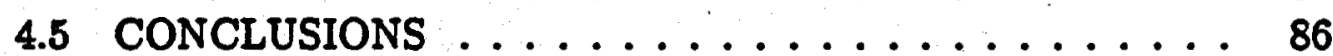

4.6 REFERENCES . . . . . . . . . . . . . 86 


\section{List of Figures}

1.1 A Typical Computational Domain . . . . . . . . 11

1.2 Irrotational Steady Flow in a Corner . . . . . . . . . . 16

1.3 Streamlines at Breakthrough for the Symmetry Element of a 5-Spot Pattern ...................... 18

1.4 Isochrones at Breakthrough for 5-Spot Pattern in Fig. 1.2 . 19

1.5 Streamlines at Breakthrough for a Staggered Line Drive $(d / a=1.0)$ 20

1.6 Isochrones for a Staggered Line Drive in Fig. $1.4 \ldots \ldots$

1.7 Schematic of the Production Scheme in a Simulated Reser-

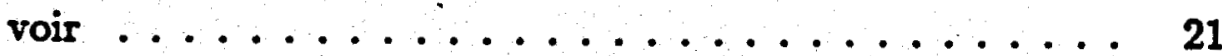

1.8 Streamlines for the Simulated Waterfood Example .... 22

1.9 Well in the Center of a Closed Square : Pressure Response at Different Locations . . . . . . . . . . . . . . 23

1.10 Pressure and Derivative Response for a Well in the Center of an Equilateral Triangle . . . . . . . . . . . 25

1.11 Two-Wells in a 2:1 Rectangle with One Short Side at Constant Pressure ....................... 26

1.12 Schematic of Elliptical Shaped Reservoirs with Different Ec-

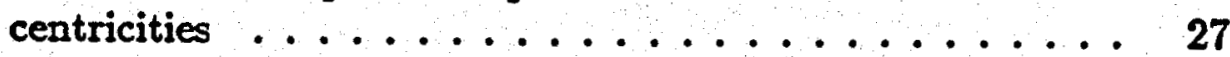

1.13 Pressure and Derivative Response for an Elliptical Reservoir 28

1.14 Schematic of a Simulated Multi-Well Reservoir . . . . . . 29

1.15 Pressure Response at Well 2 and 3 for the Simulated Reservoir ....................... 30

2.1 Fracture Flow ............... 38

2.2 Matrix Diffusion ........................ 39

2.3 Inlet Condition - Step Change ............ 42

2.4 Outlet Response to Step Change . . . . . . . . . . . 43 
2.5 Inlet Condition - Pulsed Injection . . . . . . . . . . 44

2.6 Outlet Response to Pulsed Injection . . . . . . . . . . . . 44

2.7 Chromatographic Effects of Diffusion and Adsorption . . . 47

2.8 Synthetic Tracer Breakthrough Curves . . . . . . . . . 48

2.9 Fracture Aperture Cross Plot - Approximate Equation . . . 50

2.10 Fracture A perture Cross Plot - Iterative Solution . . . . . 51

2.11 Approximate vs. Iterative Solution . . . . . . . . . 51

2.12 Well Locations in Wairakei Field (from McCabe at al, 1983) 52

2.13 Chromatographic Movement of Iodide and Bromide - Wairakei (from McCabe et al. 1983) . . . . . . . . . . . . 53

2.14 WK68 Tracer Breakthrough Curve - from WK107 . . . . 55

2.15 Fracture Aperture - Visual vs. Regression Method ... . . 57

2.16 Breakthrough Time - Visual vs. Regression Method . . . 57

2.17 Peak Concentration Time - Visual vs. Regression Method . 58

2.18 WK116 Tracer Breakthrough Curve - from WK101 . . . . 59

3.1 Model Fits to the Profile at WK108 . . . . . . . . . 70

3.2 Model Fits to the Profile at WK116 .......... 71

3.3 1-Path CD Model's Fit to the Profile at WK76 ...... 72

3.4 2-Path CD Model's Fit to the Profile at WK76 . . . . . 73

3.5 2-Path AD and AD-MD Model's Fits to the Profile at WK76 74

3.6 The Return Profile at BR20 Matched by the CD Model . . 79

4.1 Uniform matrix block size distribution response compared to single block size response . . . . . . . . . . 86 


\section{List of Tables}

1.1 Steady Irrotational Flow in a Corner . . . . . . . . . . 15

1.2 Well Flow Rates . . . . . . . . . . . . . 31

2.1 Synthetic Tracer Test - Formation Data . . . . . . . 49

2.2 Synthetic Tracer Test - Injection Data . . . . . . . . . . 49

2.3 Estimation of Fracture Aperture - Regression and Visual Methods .................... 56

3.1 Solutions to Models of Non-recirculating Flow . . . . . . 67

3.2 Solutions to Models of Recirculating Flow . . . . . . . 76 


\section{Chapter 1}

\section{Application of Boundary Element Method to Front Tracking and Pressure Transient Testing}

Jitendra Kikani and Roland Horne

\subsection{OVERVIEW}

This section presents the theory of the Boundary Integral Equation Method in general and discusses the application of this method to front tracking in steady velocity field and pressure transient testing, in arbitrarily shaped reservoirs.

Integral equation methods have existed for quite some time in the mathematical literature but have been popularized only recently in engineering applications within such diverse fields as aeronautics, heat transfer, elasticity and groundwater hydrology. Reservoir engineering applications of this method are starting to be realized.

The usefulness of the methodology lies in the fact that the solutions obtained are highly accurate and do not suffer from the usual drawbacks of the other domain type numerical schemes. Also complex reservoir geometries with multiple wells can be handled with ease due to the good boundary 
conformance obtained with the elements.

Such desirable features are realized because the analytical nature of the solution is preserved due to the use of free space Green's function of the governing differential operator as the weighting function in the weighted residual approach. A collocation type method is used for the solution of the resulting integral equations. Also, since the method is a boundary procedure, the dimensions of the problem are reduced by one. This reduction in dimensionality is obtained in cases where there are no distributed sources/sinks in the problems domain and the initial conditions are homogeneous.

The potential applications in geothermal reservoir engineering include rapid generation of streamlines and isochrones for steady state single phase flow problems. Front tracking in the steady state case can be done quickly and effectively for multi-well situations with arbitrarily shaped boundaries and a variety of boundary conditions. Application to pressure transient testing of arbitrary shaped, multi-well multi-rate reservoirs is also possible, under two different approaches.

Both the above applications are demonstrated with a variety of examples, some of which are difficult to solve by analytical means.

\subsection{INTRODUCTION}

Since the early 1800's the potential theory (Kellogg, 1953) has been used both in electrostatics and fluid mechanics. It has been known that the electrical charge within a given closed shape could be determined by knowing the charge distribution on the surface of the closed body.

However it was not until 1960's that the first application of this concept was used to solve engineering problems wherein an integral equation scheme was used to determine the potential on the bounding surface, eventually leading to the determination of the potential within the domain. Since then, and after the systematic development of weighted residual techniques (Brebbia and Walker, 1980, Connor and Brebbia, 1984), this methodology of solving partial differential equations has been gaining rapid popularity in a variety of engineering disciplines. The theory of weighted residual techniques is all encompassing and variety of numerical methods in use today are special cases of it. 


\section{Methodology}

The Boundary Integral Equation Method (BIEM), or Boundary Element Method as it is usually called, is a procedure wherein the governing linear differential equation for a particular process is cast into an integral equation form by one of the two methods:

- Expressing the differential operator in a divergence form (Zauderer, 1983);

- Using Green's second identity.

Both these techniques give identical forms. Once in the divergence form, the well known Gauss's divergence theorem is used to cast the equation into a boundary form. This reduces the dimensionality of the problem by one i.e. a three-dimensional problem is reduced to an integral around its surface and a two-dimensional problem reduces to a contour integral. If the initial conditions are non-homogeneous in an initial-boundary value problem and/or the forcing functions in the form of distributed sources are present in the system, some volume integrals remain. In such situations the interior of the domain has to be discretized for evaluation of the integrals.

The integrals that are obtained on the boundary are weighted by functions which are termed free space Green's function or fundamental solutions by various authors. Determination of this Green's function, as we shall call it, forms a major effort for the application of boundary element methodology. Green's functions for the linear differential operators that we are interested in reservoir engineering are well known and can easily be adapted to the solution by this technique.

If the weighting functions are not Green's functions which have a global support, meaning they are defined on the entire domain of the problem, but are instead some type of polynomial functions with local support and satisfy the boundary conditions, this forms the basis for the Galerkin finite element method.

In order to be able to solve for the unknown quantity inside the domain, or the reservoir in our case, one has to evaluate the integrals on the boundary of the reservoir. However, on the boundaries we only know the boundary condition. Thus, for a closed boundary we know the flux, for a constant pressure boundary we know the pressure or any combination of these is known at a particular section of the boundary. This means that 


\section{sto}

one of the two quantities (pressure or flux) is unknown. So we first need to solve the problem for the distribution of the unknown quantity on the surface before solving for the quantity within the problem domain. This leads to a collocation type scheme which is used to first obtain the unknowns on the boundary. Once the unknown boundary solutions are obtained, the interior solution can be obtained by quadrature.

Variations of this same basic method may be used for different situations. The steady state case (Laplace's Equation) has been treated by various authors (Brebbia and Waller, 1980; Liggett and Liu, 1982). In reservoir engineering applications this was considered by Numbere and Tiab (1984) and Masukawa and Horne (1985). Lafe et al (1980) discussed the handling of various types of singularities in such situations. If the nature of the singularity is known from considerations in hydrodynamics, this information can be easily introduced into a boundary element code to treat the neighborhood of these singularities in a specific manner.

Transient problems governed by the diffusivity equation have been studied by a number of researchers in heat transfer and groundwater hydrology. Rizzo and Shippy (1970) proposed a boundary element formulation for transient heat conduction problems. Pina (1984) and Taigbenu (1985) introduced various ways to handle the time stepping in the integral equation. Kikani and Horne (1988b) give a comparison of two time stepping methods, Laplace space solution and real space convolution type solution.

\section{Applicability}

The restrictions imposed by the linearity of the differential operators constrains the utility of the method. A lot of work is currently going on to relax these restrictions. At this time a linear differential operator with variable coefficients is not amenable to the simple solution procedure outlined above due to the difficulty in solving for the free space Green's function for the operator. One way to avoid this is to treat the non-linearities or the part of the differential operator that causes problems as an inhomogeneity or a forcing function. What this does is to lump all the problem terms under a domain integral. This method deviates from the concept of a purely boundary procedure because volume integrals remain in the boundary integral equation. In order to evaluate these volume integrals the whole domain has to be discretized into finite element type meshes. Another approach for steady state problems is to expand the solution in a perturbation series 


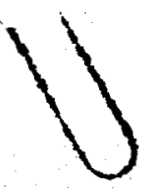

(Lafe and Chang, 1987) wherein the first term accounts for all the boundary conditions and the higher terms have homogeneous boundary conditions. Other methods like Dual reciprocity formulation (Wrobel et al, 1986) have been suggested.

Having noted the shortcomings it is only fair to look at the advantages this method has to offer. In addition to reducing the dimensionality of the problem by one, the errors due to grid orientation effects and numerical dispersion are eliminated. These errors can cause headaches in finite difference methods. The removal of the grid orientation effect follows directly from being a boundary only procedure. The numerical dispersion is minimized due to the fact that it is an integral method, which is a smoothing procedure compared to discretization or differencing which tends to accentuate the errors when evaluating derivatives. Thus, the accuracy of the solutions are much better. In the steady state case the singularities can be very easily implemented into the procedure, provided the nature of the singularities are known. Boundary element methods can be used very effectively both for streamline generation and pressure transient testing of arbitrarily shaped reservoirs with multiple wells and producing at a varying rate schedule. With the use of superposition method wellbore storage and skin can easily be added.

A general outline of the theoretical considerations for formulating a boundary element procedure is presented next. Then, specific application to steady state problems is presented. Some comparisons are made. regarding the adequacy of the interpolation functions. Next, application to pressure transient testing is presented, first with examples to compare with known analytical solutions and then with examples for cases where analytical solutions are not possible.

\subsection{THEORETICAL CONSIDERATIONS}

A typical reservoir geometry is shown in Figure 1.1. The domain of the problem is defined by $\Omega$ and the corresponding bounding surface is represented by $\Gamma$, on sections of which different types of boundary conditions could be applied. The non-dimensional diffusivity equation governing the pressure diffusion in a two-dimensional flow of a slightly compressible, single phase fluid in a homogeneous, anisotropic and bounded reservoir, with 


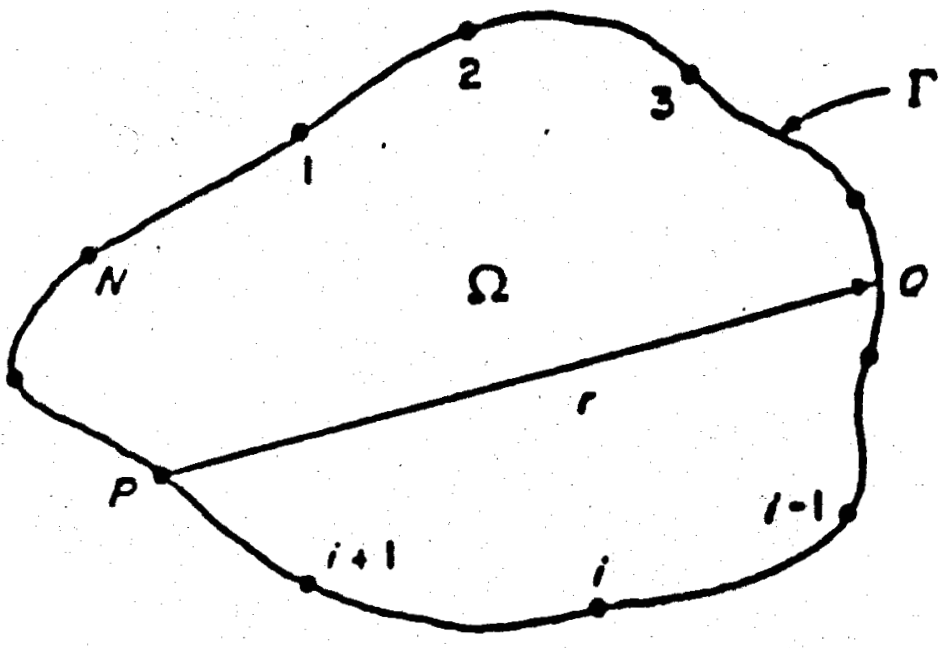

Figure 1.1: A Typical Computational Domain

$n_{w}$ individual wells, is given by (Kikani and Home, 1988b)

$$
\frac{\partial^{2} p_{D}}{\partial x_{D}^{2}}+\frac{\partial^{2} p_{D}}{\partial y_{D}^{2}}=\frac{\partial p_{D}}{\partial t_{D A}}+\sum_{i=1}^{n_{N}} Q_{D_{i}} \delta\left(x_{D}-x_{D_{i}}\right) \delta\left(y_{D}-y_{D_{i}}\right)
$$

where,

$$
Q_{D_{i}}=\frac{Q^{*} \mu A}{\rho k_{x x} \tilde{p}}
$$

and $Q_{D_{i}}$ is non-dimensionalized flow rate of the $i^{\text {th }}$ well. $Q^{*} i$ is the strength of a sink in mass per unit volume per unit time, $\tilde{p}$ is an arbitrary normalization pressure chosen to be equal to one. $\rho$ and $\mu$ are the density and viscosity of the fluid, and $k_{x x}$ is the diagonal element of the permeability tensor.

Also,

$$
\begin{gathered}
t_{D A}=\frac{k_{x x} t}{\phi \mu c_{t} A}=\frac{\eta t}{A} ; \quad p_{D}=\frac{p_{0}-p}{\tilde{p}} \\
x_{D}=\frac{x}{\sqrt{A}} ; \quad y_{D}=\frac{y}{\sqrt{A}}
\end{gathered}
$$


Since the reservoir geometry can be irregular, the above system has been normalized with respect to the area (A) of the reservoir.

Now, three types of boundary conditions can be imposed on any section of the boundary $\Gamma$. These can be :

$$
\begin{array}{cc}
p_{D}=p_{1} \quad \text { on } \Gamma_{1} \in \Gamma \quad \text { (Dirichlet) } \\
\frac{\partial p_{D}}{\partial n}=q_{1} \quad \text { on } \Gamma_{2} \in \Gamma \quad \text { (Neumann) } \\
\alpha p_{D}+\beta \frac{\partial p_{D}}{\partial n}=\gamma \quad \text { on } \Gamma_{3} \in \Gamma \quad \text { (Mixed) }
\end{array}
$$

where, $n$ is the outward pointing normal on an element on the boundary.

The Integral Equation corresponding to Eq. 1.1 can be derived by expressing the differential operator in a divergence form. For homogeneous initial conditions the equation is

$$
\begin{aligned}
& c \theta p_{D}\left(x_{D}, y_{D}, t_{D}\right)=\int_{t_{D}} d \tau \int_{\Gamma}\left(G \frac{\partial p_{D}}{\partial n}-p_{D} \frac{\partial G}{\partial n}\right) d S+\int_{t_{D}} d \tau \sum_{i=1}^{n_{D}} G_{i} Q_{D_{i}} \\
& \theta=2 \pi \quad \text { if }(x, y) \in \Omega \\
& \theta=\theta \quad \text { if }(x, y) \in \Gamma
\end{aligned}
$$

where $\theta$ is the internal angle between two boundary elements. $c$ takes different values depending on the problem and is discussed later. $G$ in Equation 1.8 corresponds to the free space Green's function. A two - dimensional free space Green's function is defined (Carslaw and Jaegar, 1959) as the pressure response at $\left(x_{D}, y_{D}\right)$ at a time $t_{D}$ due to an instantaneous line source of strength unity generated at coordinates $(\xi, \zeta)$ at the time $\tau$. The medium is initially at zero pressure and infinite in extent. If the time derivative is zero in Equation 1.1, i.e. for the two-dimensional steady state case, $\mathbf{G}$ is given by

$$
G\left(x_{D}, y_{D}, \xi, \zeta\right)=\frac{1}{2 \pi} \ln r
$$


Note that for the steady state case, the integral over time in Equation 1.8 is not present. For the transient case,

$G\left(x_{D}, y_{D}, t_{D}, \xi, \zeta, \tau\right)=\frac{1}{2 \pi\left(t_{D}-\tau\right)} H\left(t_{D}-\tau\right) \exp \left(-\frac{r_{D}^{2}}{4\left(t_{D}-\tau\right)}\right)$

where, $\mathrm{H}\left(t_{D}-\tau\right)$ is the Heaviside step function. Also,

$$
r_{D}^{2}=\left(x_{D}-\xi\right)^{2}+\left(y_{D}-\zeta\right)^{2}
$$

where, $\xi$ and $\zeta$ are the coordinates of a fictitious source point.

It is worth noting that the Green's function satisfies the material balance condition

$$
\int_{\Omega} G d A=1
$$

It can be seen from Eq. 1.12 that the Green's function for the transient problem has a convolution character when substituted in the integral Equation 1.12. This makes the solution history dependent. Thus, the solution at any time step depends on the solution at all the previous time steps. Various methods have been suggested for handling this. Taigbenu (1985) showed a variety of finite difference type time stepping schemes. An alternative way would be to treat the solution at any previous time level as the initial condition for subsequent solutions. This approach gives rise to a domain integral and the advantages of a strict boundary procedure are lost. Wrobel and Brebbia (1986) suggested a convolution treatment of the problem in a manner which requires a lot of storage in a computer implementation. Kikani and Horne (1988a) studied this in detail and found the error characteristics unreasonable for long term solution for well testing type applications, due to the accumulation of errors over successive time steps. Another method first suggested by Rizzo and Shippy (1970) is to Laplace Transform the differential equation so that the time domain only appears as a parameter. By doing this, the governing differential operator becomes the modified Helmholtz operator and the subsequent weighting function becomes,

$$
\bar{G}\left(x_{D}, y_{D}, \xi, \zeta ; s\right)=-K_{0}\left(\left|r_{D}\right| \sqrt{s}\right)
$$


The value of $c$ in the integral Equation 1.8 is one for the steady state and the transient case in Laplace space. For the transient problem in real space $c$ is two. This constant is the coefficient in the Green's function of the differential operator. The integral in the last term of Eq. 1.8 is replaced by $1 / s$, where $s$ is the Laplace space parameter.

With this methodology the solution procedure in Laplace space becomes identical to the steady state problem discussed earlier. The complication is transferred to the process of inversion back to the real space. However, with highly accurate and robust schemes available today for diffusion type of problems, very good solutions can be obtained. Further difficulty also comes about since the Green's function is now more complicated and most of the resulting integrals have to be performed numerically.

\subsection{Boundary Behavior}

Reiterating, the integral equation of Eq. 1.8 gives the pressure at any point $p_{D}\left(x_{D}, y_{D}\right)$ at time $t_{D}$ in the domain of the problem in terms of an integral over the bounding surface. The boundary integral requires knowledge of the pressure or the normal derivative of the pressure at all locations on the boundary. In a well-posed problem, either the pressure, the normal derivative of pressure or some combination of the two, is known at each point on the boundary. Thus, it is imperative to first solve for the unknown boundary condition and hence the integral equation (which is a Fredholm integral equation of first or second kind depending on the unknowns).

Equation 1.8 is solved for the unknown boundary condition by choosing a discrete number of elements on the boundary. An interpolating function for pressure and flux (or normal derivative of pressure) on a boundary element both in space and time dimensions is assumed. The integral equation can be solved by a collocation type technique by moving a fictitious source point (or Green's function source) to all the nodes and generating enough equations so that they match the number of unknowns. The problem then reduces to one of solving a matrix equation. Once both pressure and normal derivative of pressure are known at all points on the boundary, Eq. 1.8 is used again to obtain the solution at any internal point.

Various kinds of interpolating functions and boundary shape functions can be used between nodal elements. Constant, linear and parabolic in- 
Table 1.1: Steady Irrotational Flow in a Corner

\begin{tabular}{|l|c|c|}
\hline Elements & Node Points & Maximum \% Error \\
\hline Constant & 12 & 7.9 \\
Linear & 13 & 0.94 \\
Linear & 25 & 0.061 \\
\hline \multicolumn{3}{|c}{} \\
\hline \multicolumn{3}{|c|}{ Numbere (1982) }
\end{tabular}

terpolation functions have been used before. We have chosen interpolating functions between boundary elements that are linear in space. Higher order interpolating functions could be chosen if the problem so requires, but usually with higher order elements all the integrals that arise have to be done numerically. Justification for using linear functions instead of constant functions is presented later.

\subsection{STEADY STATE PROBLEMS}

The governing equation is given by Eq. 1.1 with the time derivative zero. Examples of situations where this equation applies are in injection front tracking for tracer flow, or at late times in pressure transient testing in a bounded system with constant pressure outer boundaries.

As mentioned earlier, in order to solve for the unknown boundary conditions, interpolation functions between boundary nodes have to be specified. Numbere (1982) used constant interpolation functions whereas we use linear interpolation functions. Figure 1.2 shows a schematic of an irrotational flow through a corner. Dimensionless pressures of 10 and 5 are maintained respectively at the two adjacent sides of the square domain. Note that due to a sudden pressure change at the upper right hand corner, the flux is infinite at that point. The analytical solution for this problem can be obtained fairly easily. A comparison is made in Table 1.1 between the analytical solution, the solution with constant boundary elements and the solution with linear elements. Clearly the linear elements give superior results even in. cases where singularity exists. Note also that the constant elements avoid the actual location where singularity exists because the pressure or flux is evaluated at the midpoint of the elements. 


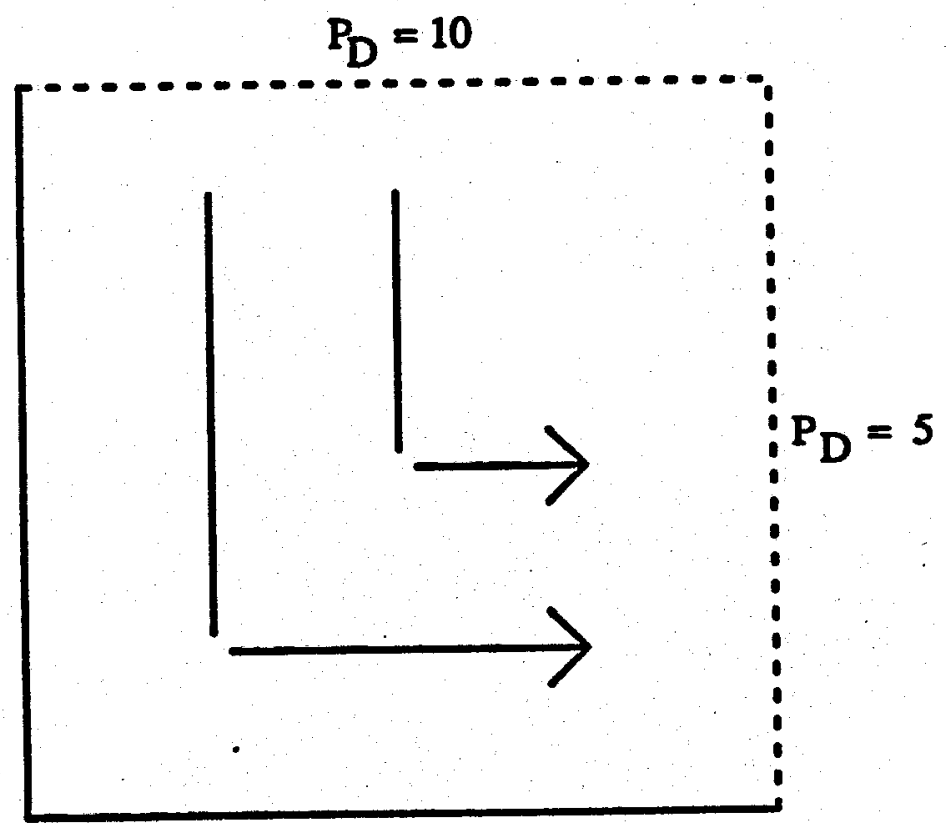

Figure 1.2: Irrotational Steady Flow in a Corner 


\section{Treatment of Singularities}

Singularities in flow situations occur when the flux or the pressure at certain location goes to a mathematical infinity. Sources and sinks with diminishing radii are examples of such singularities. Also sharp bends and corners in the flow geometry cause the flow behavior to become singular. The singularities can be treated in two different ways. Well singularities can be treated as inhomogeneities in the flow equation. Being Dirac-delta functions they appear only as additive term as shown in Eq. 1.1. Another way to treat them is to subtract of the contribution of the singularity from the governing equation and the boundary condition. Then the singularity free problem is solved with the conventional boundary element procedure (Liggett and Liu, 1984). Once the solution is obtained, the contribution of the source/sink is added back in. The nature of some of the singularities which occur due to bends and corners in the flow path are known from hydrodynamics. These can be subtracted off from the boundary elements which are in the vicinity of the singularities. This very simple procedure improves the accuracy of the solutions tremendously.

\section{Streamline generation and Front tracking}

Since sources and sinks are so simple to include and the handling of complex geometries is simplified, the boundary element method could be used effectively for generating streamlines in multi-well complex geometry situations. It is fast and accurate because the dimensionality of the problem is reduced by one.

Figure 1.3 shows the streamlines in the symmetric element of a fivespot pattern. The results compare very well with the analytical solution presented by Morel-Seytoux. Fig. 1.5 depicts the isochrones or the location of fluid particles at a particular time after the start of injection. This is essentially the location of the front. Figs. 1.5 and 1.6 show the streamlines and isochrones for the symmetric element of a staggered line drive pattern which has a d/a ratio of 1.0. Cumulative recovery and incremental recovery until breakthrough is very simply calculated. This technique is not limited to symmetric well patterns but could be used as efficiently for reservoirs with arbitrary geometry. An example of such a case is shown by a schematic in Figure 1.7. There are 4 producers and 2 injectors in a balanced tracer injection scheme. Well number 2 and 5 inject at a rate 2q $\mathrm{bbl} /$ day and the other four wells produce fluids at a rate of $\mathrm{q}$ bbl/day. Fig- 


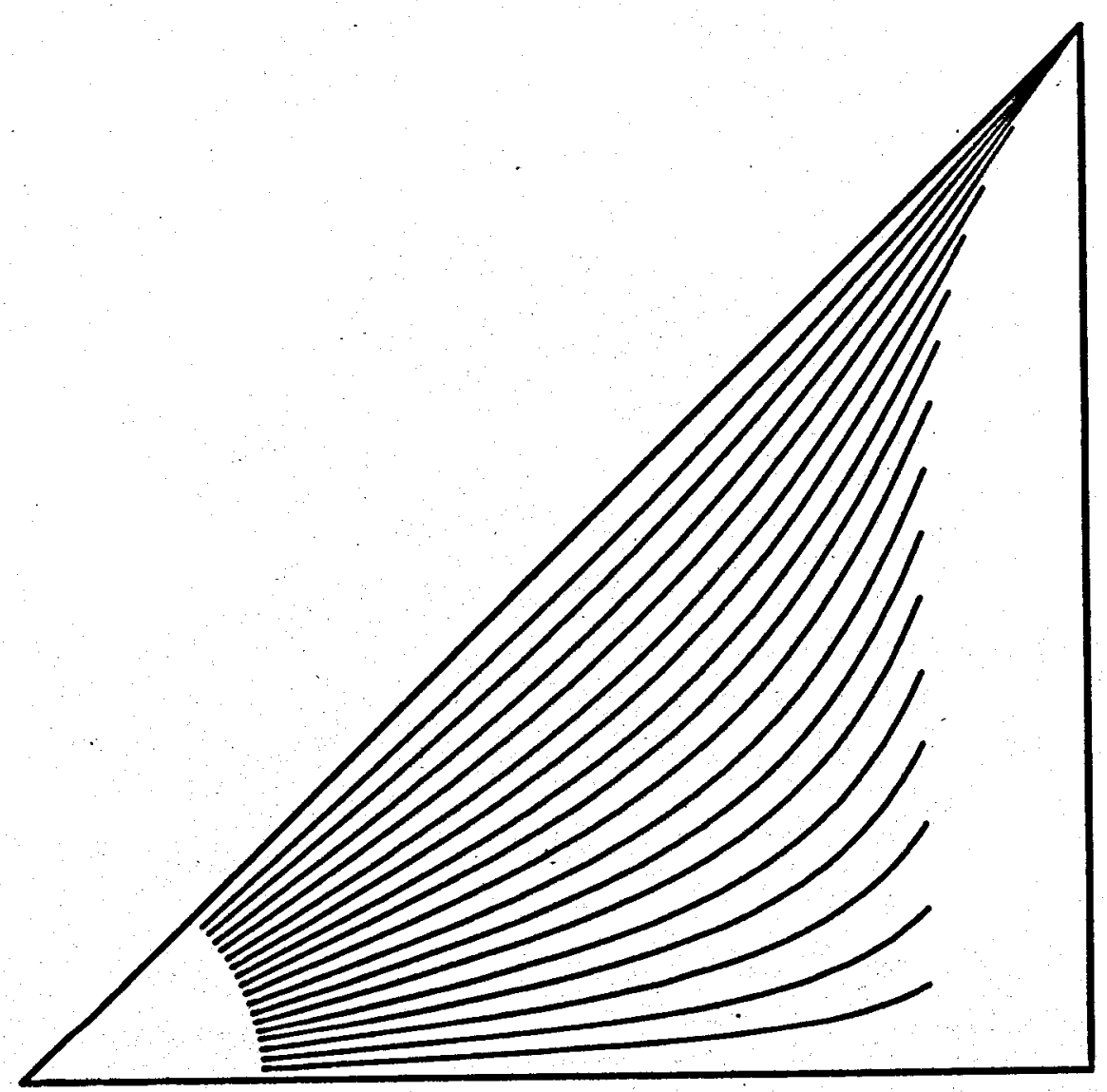

Figure 1.3: Streamlines at Breakthrough for the Symmetry Element of a 5-Spot Pattern 


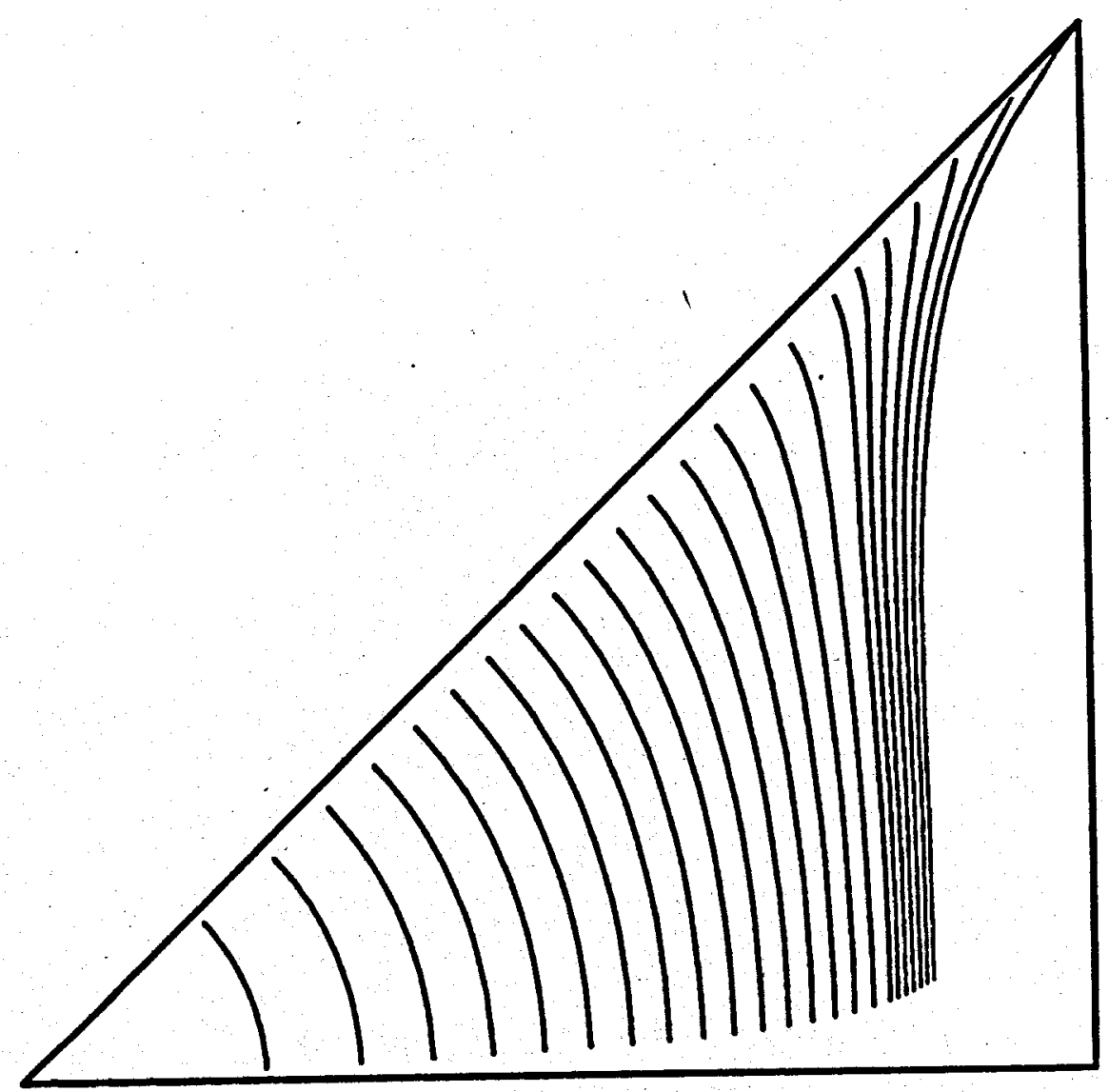

Figure 1.4: Isochrones at Breakthrough for 5-Spot Pattern in Fig. 1.2 


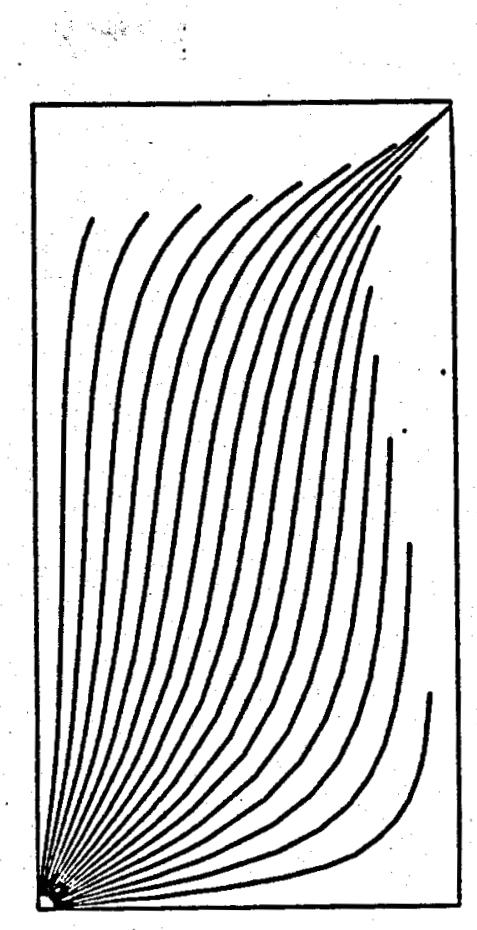

Figure 1.5: Streamlines at Breakthrough for a Staggered Line Drive $(d / a=1.0)$

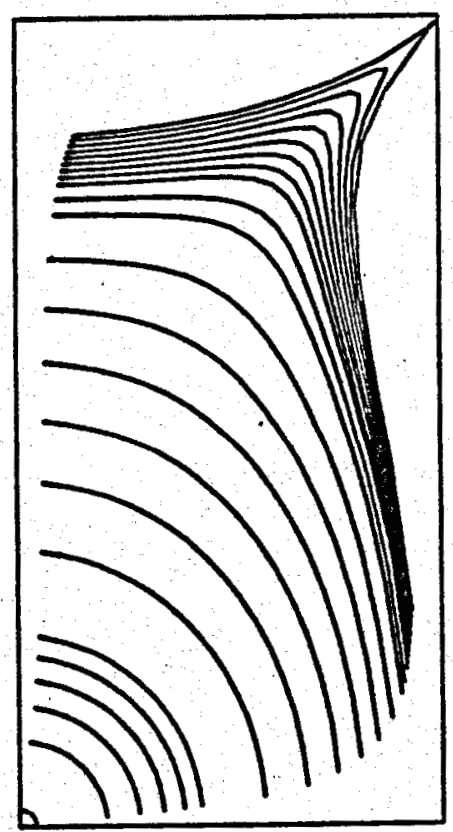

Figure 1.6: Isochrones for a Staggered Line Drive in Fig. 1.4 


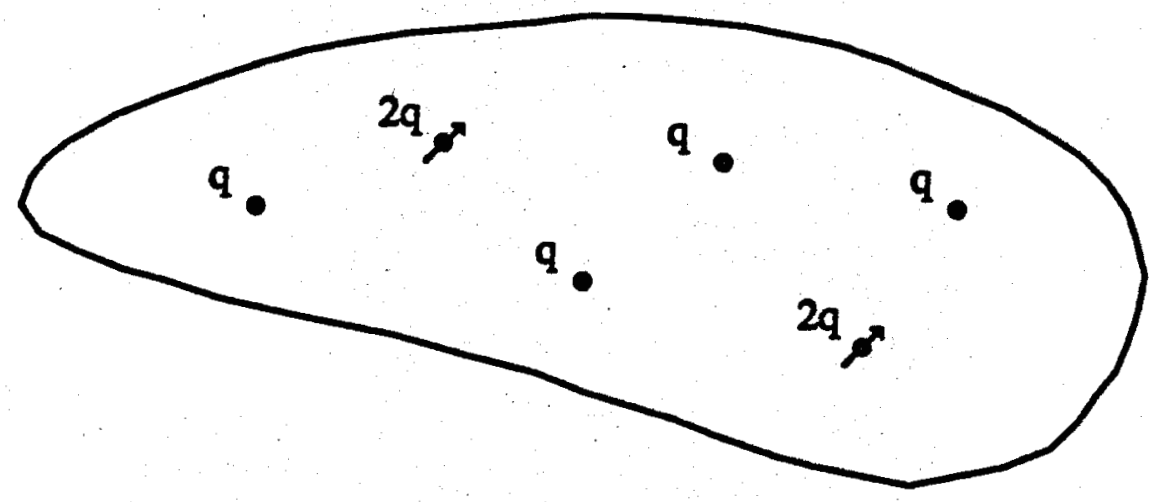

Figure 1.7: Schematic of the Production Scheme in a Simulated Reservoir

ure 1.8 shows the streamlines for this reservoir generated by the boundary element technique. The streamlines that reach the well are stopped from further movement. The internal solution generation is very similar to a particle tracking technique once the boundary solution has been obtained.

\subsection{UNSTEADY STATE PROBLEMS}

The governing equation is given by Eq. 1.1. This problem can be solved in a variety of ways, especially considering the way the time stepping is handled. When solved in real space, convolution integrals result which are tedious to evaluate. Due to the availability of robust numerical inversion techniques it is quite convenient to implement the whole procedure in Laplace space, as discussed earlier. We will only refer to the Laplace space BEM from now on. With linear interpolation functions in space, most of the integrals had to be evaluated numerically owing to the complexity of the weighting functions. However, this was not a disadvantage because for the monotonically decreasing functions these integrals can be evaluated very accurately by Gaussian panel type integration schemes.

The coefficient matrix obtained is completely full, unlike many other 


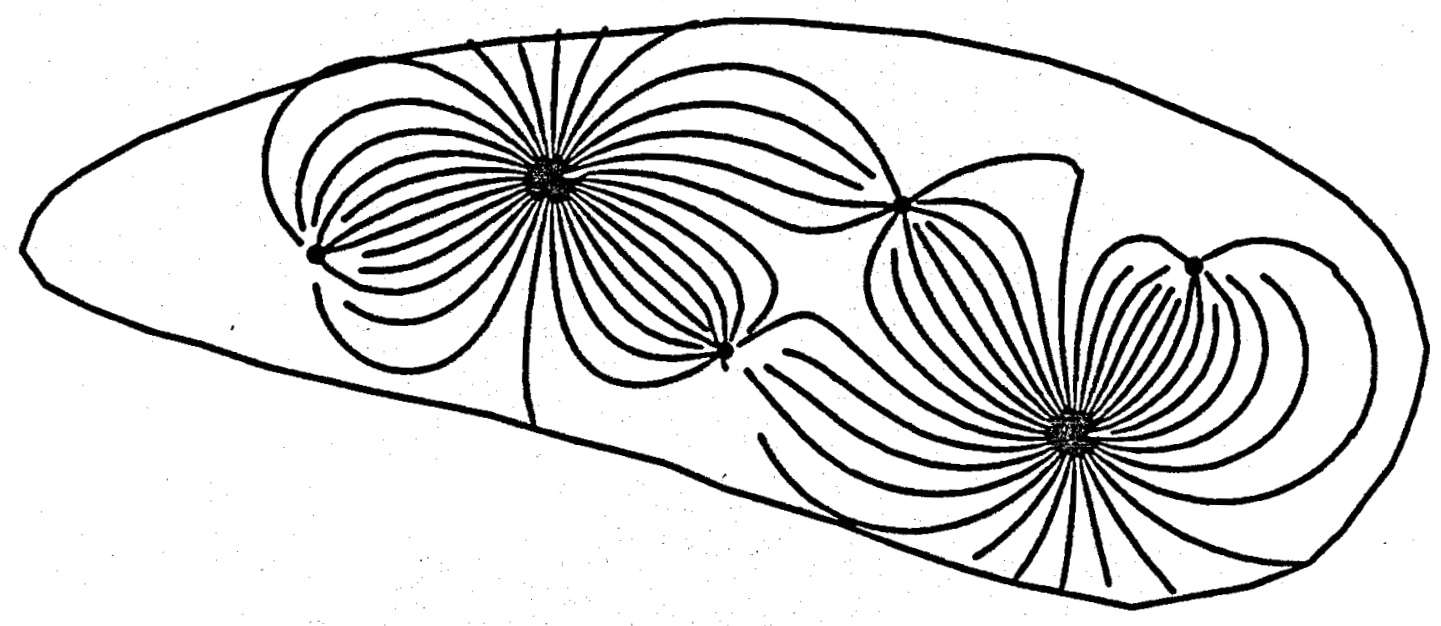

Figure 1.8: Streamlines for the Simulated Waterflood Example

numerical methods which give rise to sparse and banded matrix structures. The reason for the full matrix is that the Green's function for the governing differential operator has a global support as opposed to local support for the interpolation functions as in other numerical schemes. Thus, the matrix is more time consuming to invert, but since this is a boundary method fewer nodes are needed for highly accurate solutions.

Some pressure transient problems of interest were solved by this method. The first examples considered were problems which have analytical solutions and are taken from the petroleum engineering literature. Figure 1.9 is a plot of dimensionless pressure versus dimensionless time based on the area of the reservoir for a well in the center of a closed square (Earlougher, 1977). The area of the reservoir is taken to be $10^{6} \mathrm{ft}^{2}$. Pressures are computed at the wellbore represented by the coordinates $(0,0)$ and various interference points. The solid lines denote the analytical solutions obtained by the superposition of the image wells. The boundary element method does an excellent job of matching the pressures. The errors are less than $0.5 \%$ when 16 boundary nodes are used. Since the advent of very accurate pressure gauges for pressure measurements in well tests, the pressure derivative technique for analysis of the data has become very prominent. 


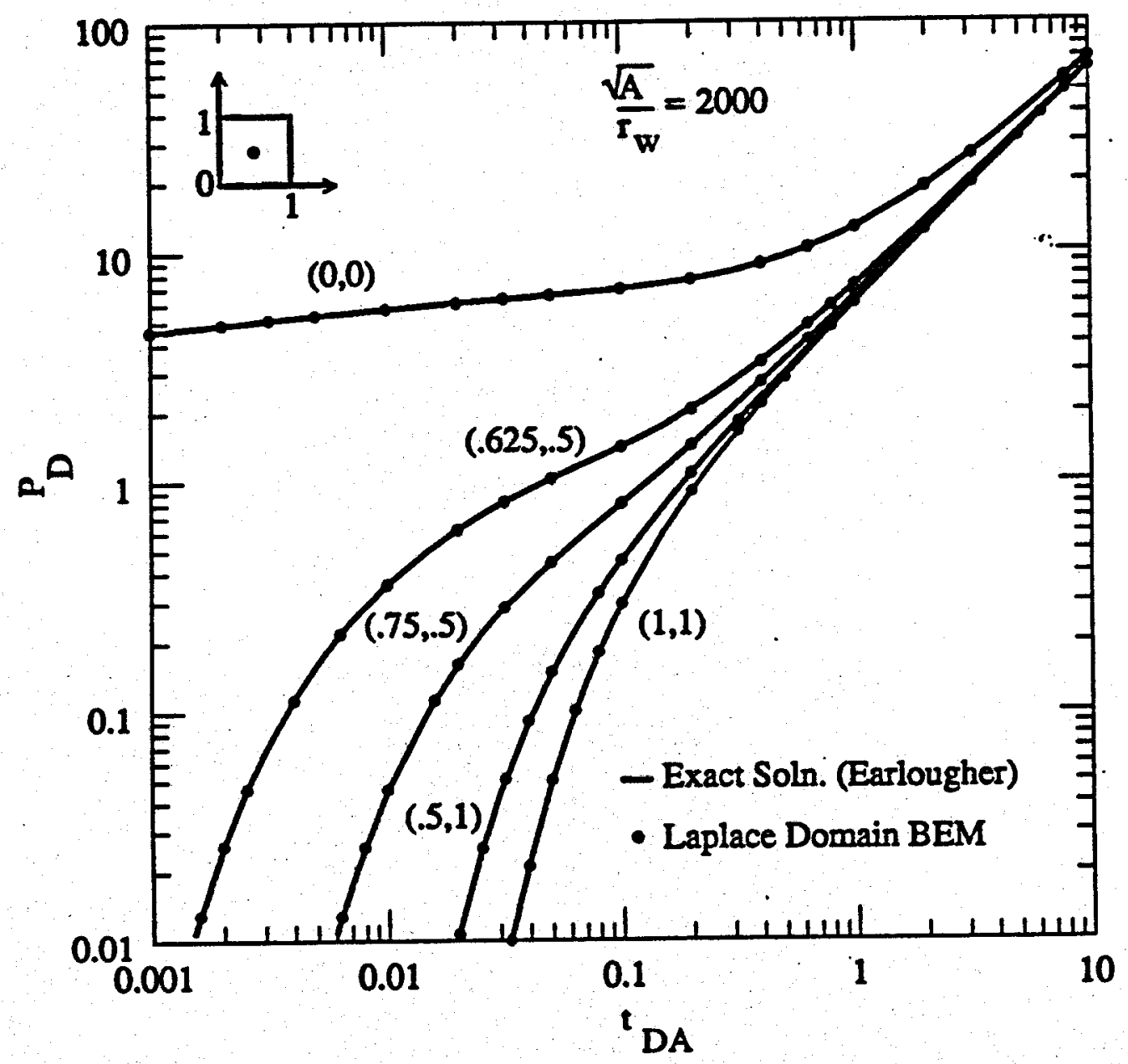

Figure 1.9: Well in the Center of a Closed Square : Pressure Response at Different Locations 
Figure 1.10 shows the $\log -\log$ plot of pressure and the semi-log pressure derivative of a well producing at a constant rate from the center of a closed equilateral triangle of size $A / r_{w}^{2}=4 \times 10^{6}$. The semi-log pressure derivative is given as follows.

$$
P_{D}^{\prime}=\frac{d P_{D}}{d \ln t_{D A}}=t_{D A} \frac{d P_{D}}{d t_{D A}}
$$

The pressure derivative analysis accentuates each of the flow regimes and thus aids in precise identification of flow regimes and parameters. The solution matches well with the analytical solution given by the solid lines. Fig. 1.11 shows a multi-well situation with two wells producing from within the rectangle with one short side at constant pressure. The pressure is evaluated at the well closer to the constant pressure boundary. The pressure derivative graph shows that the system tries to go to pseudosteady state but then the constant pressure boundary dominates and the derivative eventually goes to zero. The pressures are compared to the analytical solutions given by Ramey et al (1973). Excellent agreement is obtained.

It is important that with such a solution procedure the consistency of the solution be checked. Increasing the number of boundary nodes does make the solution more accurate for the cases studied above. Kikani and Horne, (1988b) illustrate this check for a well in the center of a closed square.

Figure 1.12 is an illustration of a well in the center of a closed elliptical shaped reservoir. Though of the same area, the eccentricities of the two ellipses shown are different. Figure 1.13 shows the pressure and pressure derivative response at the wellbore for the elliptical system of Figure 1.12. As expected, after the early infinite acting period, characterized by a slope of 0.5 , there is a short transition before the external boundaries are felt and the system goes to pseudosteady state shown by a slope of one on the log-log plot of semi-log pressure derivative. The early and late time pressure derivative behavior for both eccentricities is the same. At intermediate transition times the system with larger eccentricity deviates quicker from the semi-log straight line and has a longer transition before getting to pseudosteady state.

The next example demonstrates the flexibility of pressure transient solutions that can be obtained by the boundary element method. Figure 1.14 shows a schematic of a production scheme in a reservoir with two parallel 


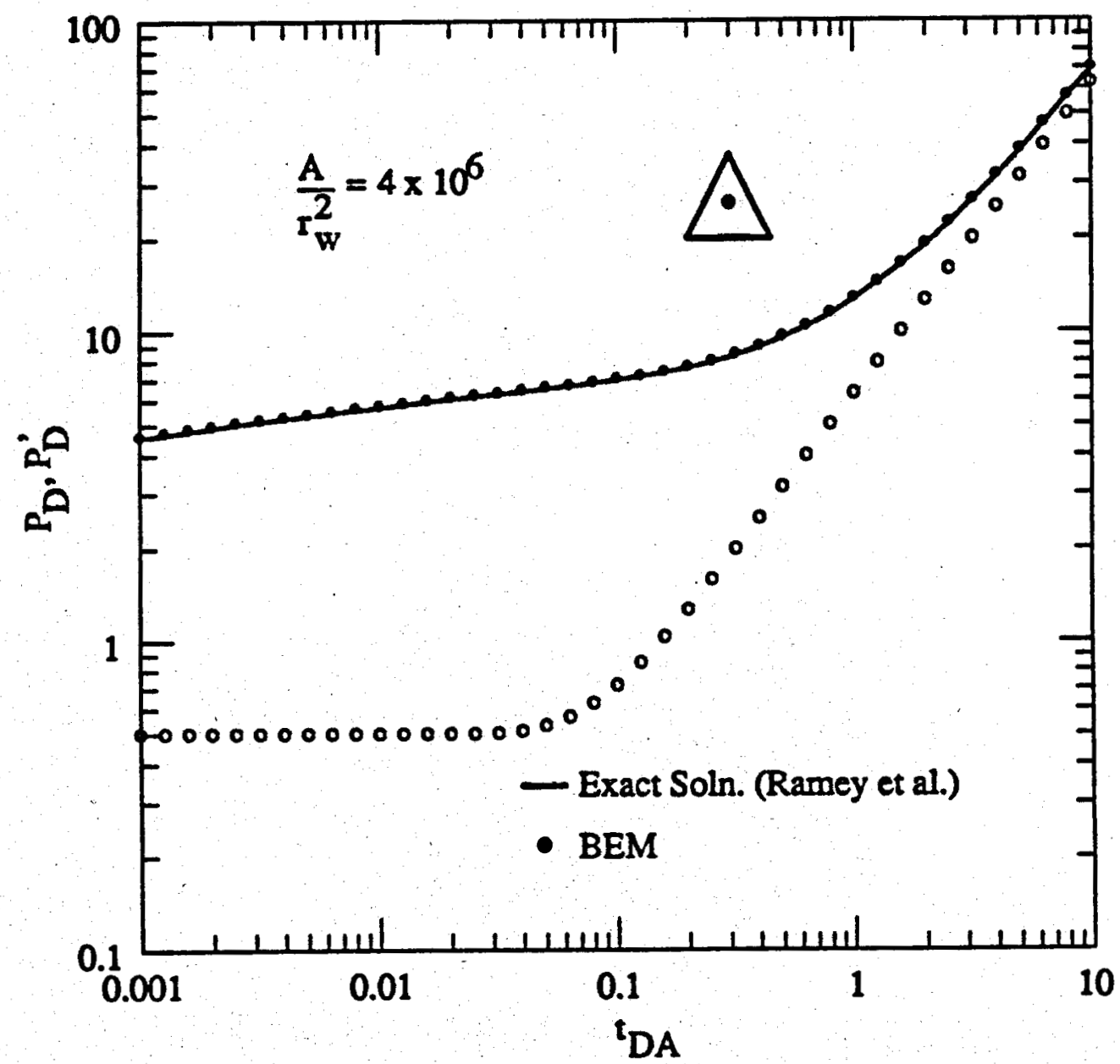

Figure 1.10: Pressure and Derivative Response for a Well in the Center of an Equilateral Triangle 


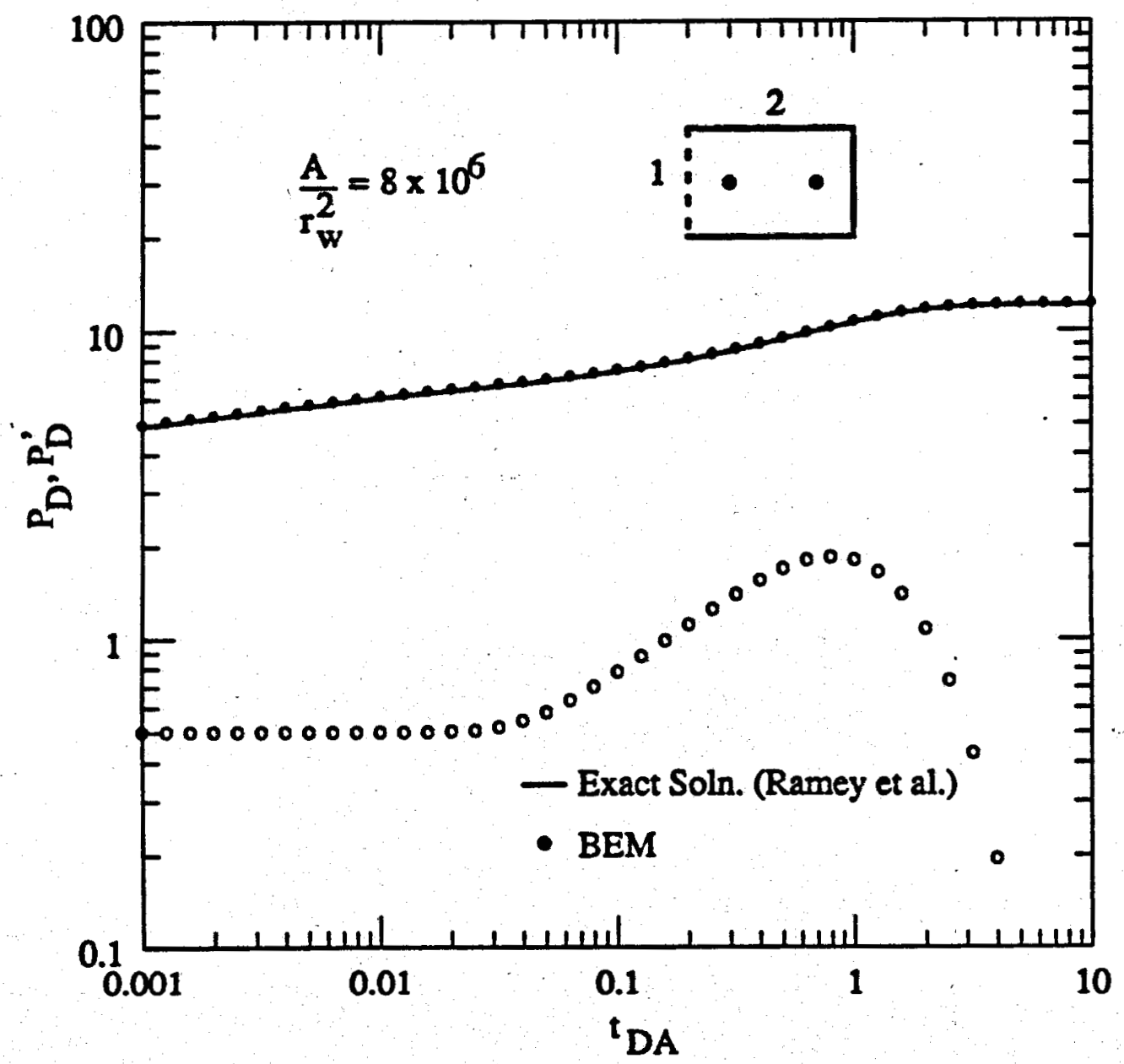

Figure 1.11: Two-Wells in a 2:1 Rectangle with One Short Side at Constant Pressure 


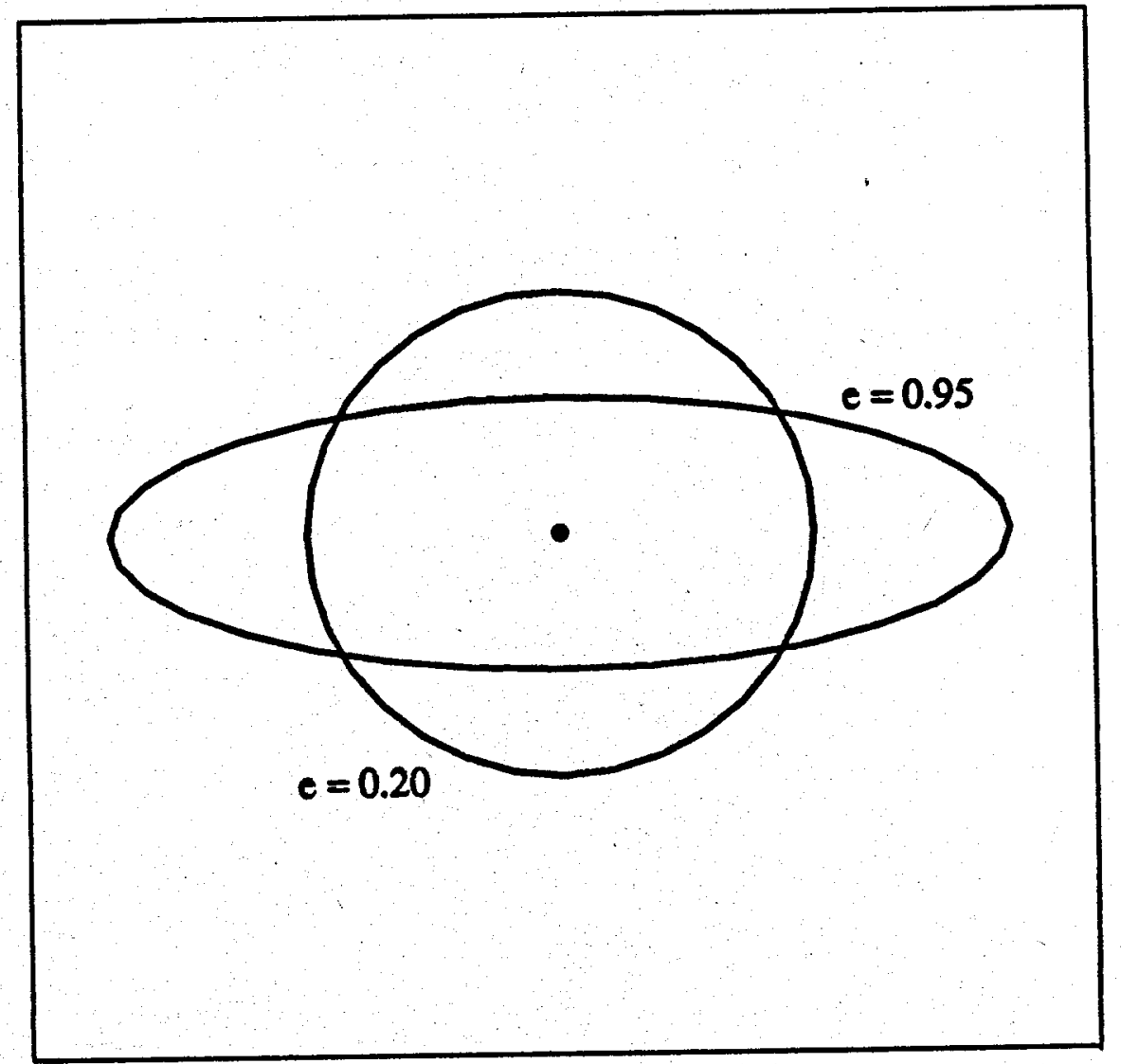

Figure 1.12: Schematic of Elliptical Shaped Reservoirs with Different Eccentricities 


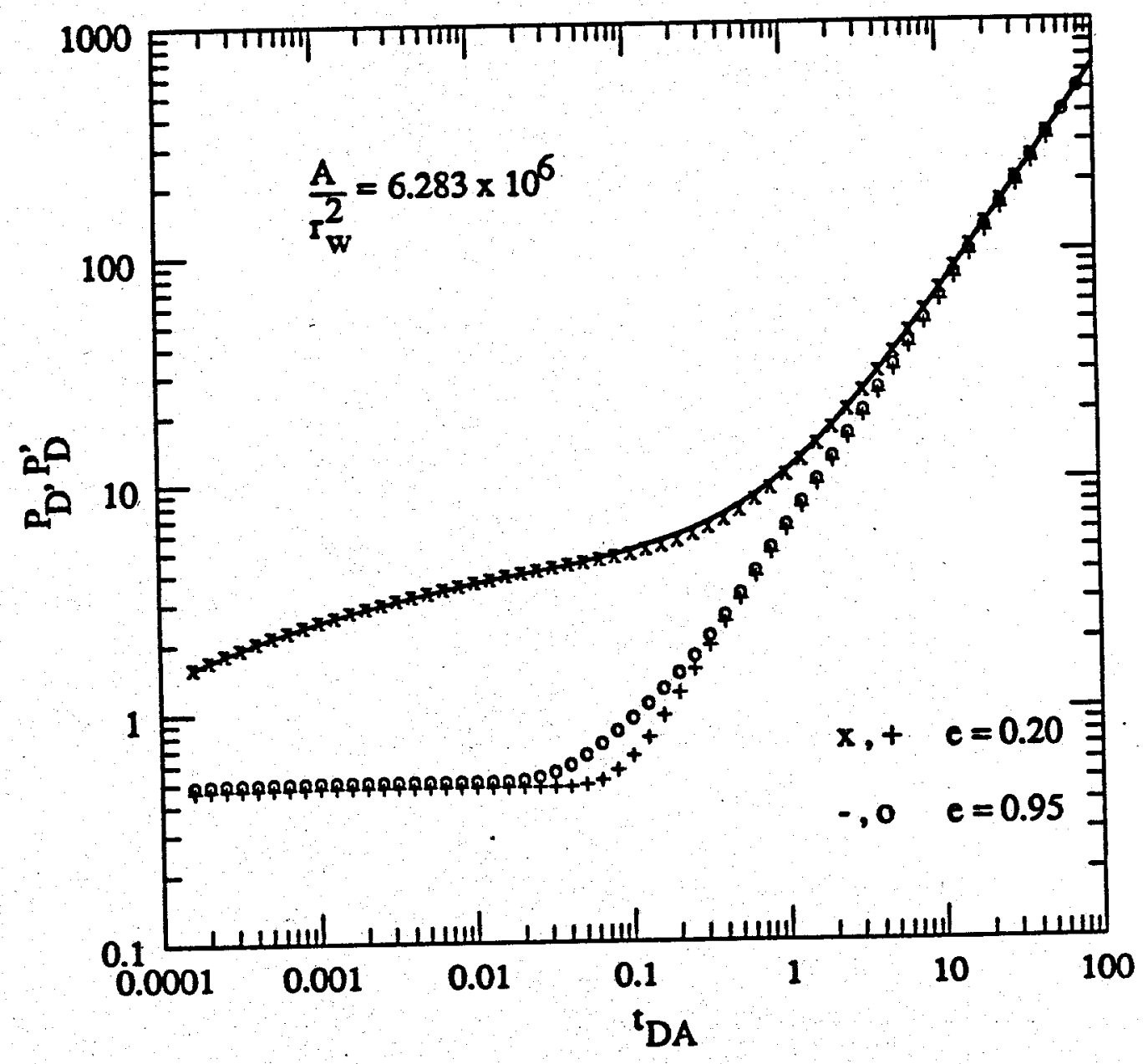

Figure 1.13: Pressure and Derivative Response for an Elliptical Reservoir 


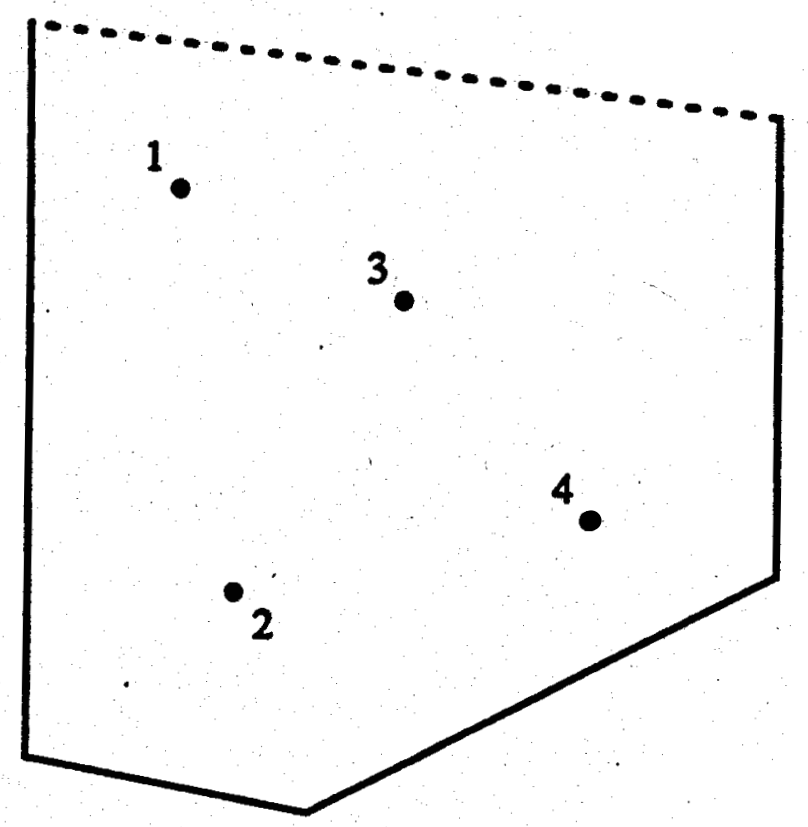

Figure 1.14: Schematic of a Simulated Multi-Well Reservoir

sealing faults and bottom igneous rock allowing no flow and a constant pressure boundary. There are 4 wells producing in the system. The injection and production rate of the wells are given in Table 1.2. Figure 1.15 shows the drawdown pressure transient and pressure derivative response in well 2 and well 3 respectively which is effected by the presence of the background trend owing to the production and injection in the rest of the wells. The presence of a constant pressure boundary makes the solution go to steady state at late times. At intermediate times, Well 2 starts going to pseudosteady state when it feels the closed boundaries around it, but later the steady state boundary is felt and the derivative dips and goes to zero. The convenience of simulating pressure response under such situations may be one of the most attractive attributes of the boundary element method, since these solutions cannot be achieved easily with equivalent accuracy by any of the traditional numerical schemes. 


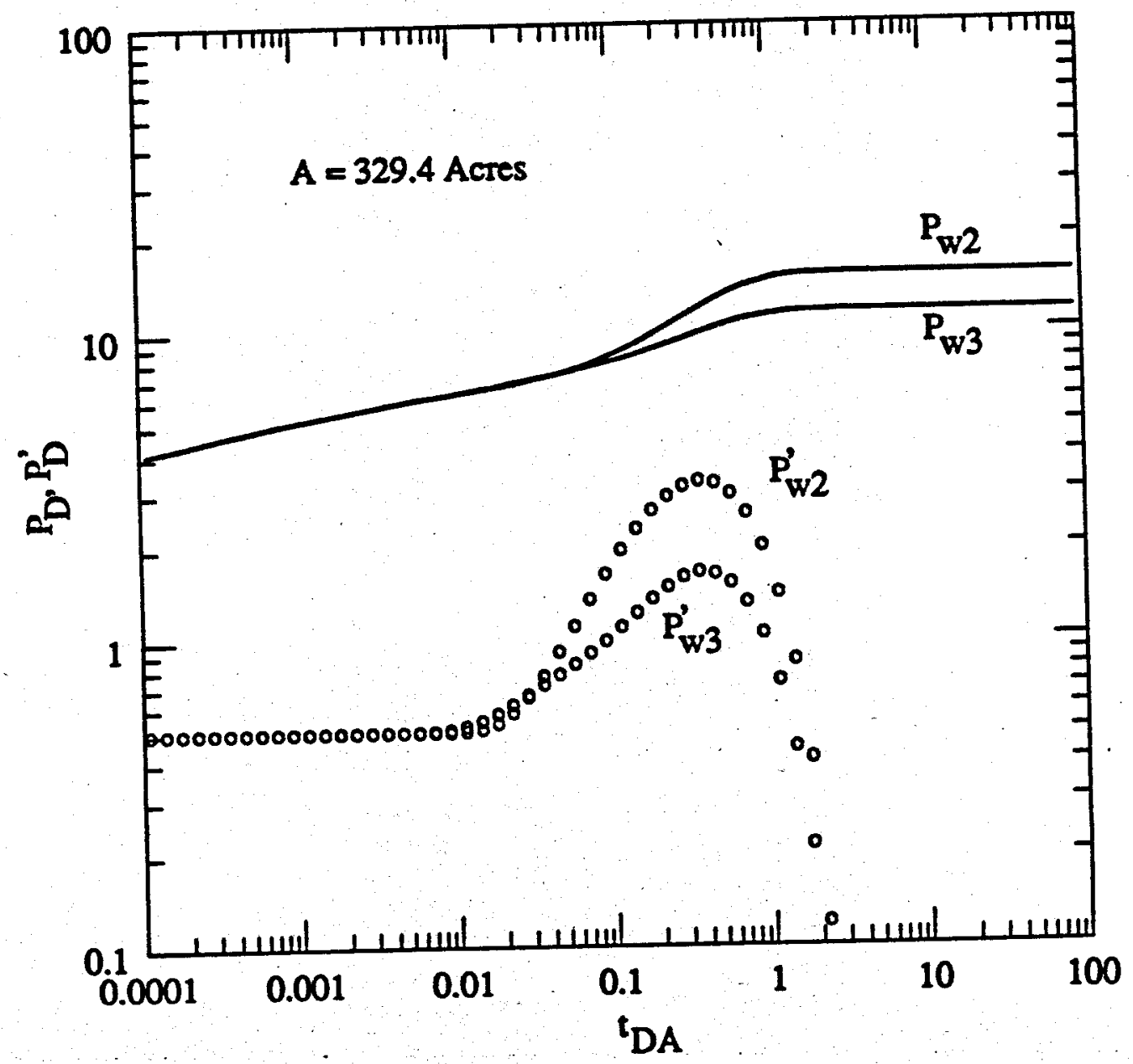

Figure 1.15: Pressure Response at Well 2 and 3 for the Simulated Reservoir 
Table 1.2: Well Flow Rates

\begin{tabular}{|c|c|}
\hline Well Number & Flow Rate $\left(Q_{D}\right)$ bpd \\
\hline 1 & 150 \\
2 & 300 \\
3 & 300 \\
4 & 100 \\
\hline
\end{tabular}

\subsection{LIMITATIONS}

With the advent of sophisticated tools for reservoir characterization, variable permeability and porosity fields can be ascribed to a reservoir. The boundary element method is limited currently to handle situations with uniform or at most sectionally non-uniform permeability fields. Work needs to be done to accommodate permeability variations. It does not appear to be a trivial task at this stage. Also, in order to enhance the applicability of boundary element method as a general simulation tool, it is desirable that non-linearities be handled. This is not directly possible because of the need to have a linear differential operator in order to define the Green's function for the problem. It might be worthwhile looking into ways to obtain approximate Green's function for non-linear operators in a perturbation sense.

The limitations of the method are noted here in order to encourage research in this direction as this methodology may prove to be useful in other geothermal reservoir engineering applications.

\subsection{CONCLUSIONS}

Boundary Element formulations for both steady state and transient single phase fluid flow through homogeneous, anisotropic porous media is presented. Various examples are shown to prove the usefulness of this technique in streamline generation in the steady state case and simulation of pressure transient response for arbitrary shaped multi-well situations. In addition to the reduction in dimensionality obtained by casting the govern- 
ing differential equation in boundary integral form, very accurate solutions are obtained by this method. Advantages and limitations are discussed and areas of further research are identified.

\subsection{NOMENCLATURE}

$$
\begin{aligned}
& A=\text { Area of the problem domain } \\
& G(x, y, t, \xi, \eta, \tau)=\text { Free Space Green's Function, (two point function) } \\
& H(t-\tau)=\text { Heaviside step function, } 0 \text { if } t \tau, 1 \text { if } t \geq \tau \\
& K_{0}(x)=\text { Modified Bessel function, second kind, order zero } \\
& Q^{*}=\text { Flow rate of a well, }\left[\mathrm{ML}^{-3} T^{-1}\right] \\
& c=\text { Constant } \\
& c_{t}=\text { Single phase fuid compressibility } \\
& k_{x x}, k_{y y}=\text { Principal directions of permeability } \\
& n=\text { Outward pointing normal to a line segment } \\
& p=\text { Pressure, psia. } \\
& x, y=\text { Variables in Cartesian coordinate system } \\
& r=\text { Distance Vector } \\
& s \text { = Laplace transform parameter } \\
& t=\text { Time } \\
& \Omega=\text { Domain of the solution region } \\
& \Gamma=\text { Boundary of the solution region } \\
& \alpha, \beta, \gamma=\text { constants } \\
& \delta(x-\xi)=\text { Dirac-delta function, singular at } x=\xi \\
& \delta_{i j}=\text { Kronecker delta, } 0 \text { if } i \neq j \text {, and } 1 \text { if } i=j \\
& \theta=\text { Angle subtended between adjacent elements, Eq. } 1.8 \\
& \xi, \zeta=\text { Local coordinates based on fictitious source point } \\
& \eta=\text { Diffusivity }=\frac{k_{s r}}{\phi \mu \omega_{t}} \\
& \phi=\text { Porosity } \\
& \epsilon=\text { Belongs to, or is an element of } \\
& \mu=\text { Viscosity, cp } \\
& \rho=\text { Density, gm./cc. }
\end{aligned}
$$

Subscripts 


$$
\begin{aligned}
D & =\text { Dimensionless } \\
D A & =\text { Non-Dimensionalized with respect to Area } \\
1,2,3 & =\text { Sections of the boundary } \\
x, y & =\text { Partial derivative with respect to } x \text { or } y \\
t & =\text { Partial derivative with respect to } t
\end{aligned}
$$

\subsection{REFERENCES}

1. Brebbia, C.A., Walker, R.H., : Boundary Element Techniques in Engineering, Newnes - Butterworths, London (1980).

2. Carslaw, H.S., Jaegar, J.C., : Conduction of Heat in Solids Clarendon Press, Oxford, second ed. (1959).

3. Connor, J.J., Brebbia, C.A., : "Boundary Element Methods," in Boundary Element Methods in Computer Aided Engineering, Ed. Brebbia, C.A., Martinus Nijhoff Publishers, Dodrecht, (1984).

4. Earlougher, R.C.,Jr., : "Advances in Well Test Analysis," Monograph vol. 5, Society of Petroleum Engineers of AIME, Dallas, (1977), 18691.

5. Kellogg, D.O., : Foundations of Potential Theory, Dover Publications Inc., New York (1953).

6. Kikani, J., Horne, R.N., : "A Convolution Boundary Element Method for Unsteady State Groundwater Flow in Homogeneous Aquifers," Proc. XIII Geothermal Res. Eng. Workshop, Stanford U., Stanford, (Jan. 19-21, 1988a).

7. Kikani, J., Horne, R.N., : "Pressure Transient Analysis of Arbitrary Shaped Reservoirs with the Boundary Element Method," SPE 18159 presented at the $63^{\text {rd }}$ Annual Technical Conference and Exhibition, Houston, Texas, (Oct. 1-4, 1988b).

8. Lafe, O.E., Cheng, A.H-D., : "A Perturbation Boundary Element Code for Steady State Groundwater Flow in Heterogeneous Aquifers," Water Resources Research, vol. 23, no. 6, (June, 1987) 1079-1084. 
9. Lafe, O.E., Montes, J.S., Cheng, A.H-D., Liggett, J.A., Liu, P.L-F., : "Singularities in Darcy Flow Through Porous Media," J. Hyd. Div., Proc. of the ASCE, vol. 106, no. HY6, (June, 1980) 977-97.

10. Liggett, J.A., Liu, P.L-F, : "Unsteady Flow in Confined Aquifers: A Comparison of Two Boundary Integral Methods," Water Resources Research, vol. 15, no. 4, (Aug. 1979) 861-66.

11. Liggett, J.A., Liu, P.L-F., : The Boundary Integral Equation Method for Porous Media Flow, George Allen and Unwin, London, (1983).

12. Liu, P.L-F., Liggett, J.A., : "Boundary Integral Solutions to Groundwater Problems," Proc. First Int. Conf. on App. Num. Modeling, U of Southampton, England, (July 11-15, 1977) 559-69.

13. Masukawa, J., : "The Application of The Boundary Integral Method to Immiscible Displacement Problems" M.S. Report, Stanford University, Stanford (June, 1985).

14. Morel-Seytoux H.J., : "Analytical-Numerical Method in Waterflooding Predictions," Soc. Pet. Eng. J. (Sept., 1965)

15. Numbere, D.T., : A General Streamline Modeling Technique for Homogeneous and Heterogeneous Porous Media with Applications to Steamflood Prediction," Ph.D Dissertation, University of Oklahoma, Norman (1982).

16. Pina, H.L.G., : "Time Dependent Potential Problems," in Boundary Element Methods in Computer Aided Engineering Ed. Brebbia, C.A., Martinus Nijhoff Publishers, Dodrecht, (1984).

17. Ramey, H.J.Jr., Kumar, A., Gulati, M.S., : Gas Well Test Analysis Under Water Drive Conditions, AGA Press, Arlington, 1973.

18. Rizzo, F.J., Shippy, D.J., : "A Method of Solution for Certain Problems of Transient Heat Conduction," AIAA Journal, vol. 8, no. 11, (Nov. 1970) 2004-2009.

19. Stehfest, H., : "Algorithm 968 , Numerical Inversion of Laplace Transforms [D5]," Communications of ACM, (Jan, 1970), vol. 13, No. 1, 47-49. 
20. Taigbenu, A.E., : "A New Boundary Element Formulation Applied to Unsteady Aquifer Problem, "Ph.D Thesis, Cornell University, Ithaca (1985).

21. Wrobel, L.C., Brebbia, C.A., Nardini, D., : "The Dual Reciprocity Boundary Element Formulation for Transient Heat Conduction," Proc. of the Sixth Int. Conf. on Finite Elements in Water Resources, Lisbon, Portugal, (1986) 801-811.

22. Zauderer, E., : Partial Differential Equations in Applied Mathematics, Wiley-Interscience, New York, (1983). 


\section{Chapter 2}

\section{Determination of Fracture Aperture - A Multi-Tracer Approach}

Charles E. Fox and Roland N. Horne

\subsection{OVERVIEW}

This work shows that fracture aperture can be calculated from tracer tests involving two tracers with different affinities for adsorption onto the rock. In 1983 Jensen proved that fissure width can be determined when adsorptive effects are neglected. Adsorption introduces an additional unknown, the retardation factor, into the governing equations. When two tracers are injected, the number of available equations expands to match the number of unknowns.

This report also presents equations based on the matrix diffusion model which allow estimation of fracture aperture through visual examination of tracer tests. Tracer data from tests in the Wairakei geothermal field in New Zealand were analyzed by the visual method. The results were compared to computations using nonlinear regression. The two analyses differed randomly with an average variation of $\pm 40 \%$. 


\subsection{INTRODUCTION}

The disposal of produced water is an important part of geothermal development. Often the water contains pollutants which preclude dumping into the biosphere. Reinjection of waste fluids into the productive formation is normally the most economical solution. Injection programs can have positive effects such as pressure maintenance and the replacement of reservoir fluid. Conversely the introduction of cooled fluids may damage the productivity of the reservoir. Cold water could flow into production wells and decrease thermal recovery.

Geothermal fields are especially prone to breakthrough problems because the primary flow paths are through fractures. Cooled fluids can short circuit the low permeability matrix and flow quickly through the fissures from the injector to the producer. The prediction of the arrival of the cold front is therefore of considerable economic importance.

The process of heat transfer between fluids and heat sources has been extensively studied. Solutions are readily available for the flow of fluids through heat exchangers of almost any configuration. The major problem for the geothermal engineer is to define the geometry of the system since the other parameters such as heat transfer coefficients are known.

Recently the matrix diffusion model has been developed to describe the fiow of tracers or contaminants through fractured porous media. The use of this model is extended in this report to cover the determination of fracture aperture from tracer tests in which adsorption is significant. Once this parameters is known the estimation of thermal breakthrough is straight forward.

\subsection{THEORY}

As stated in the introduction, many authors have utilized the matrix diffusion model to study the flow of tracers through fractures. Contributions of this work include the derivation of a fracture aperture equation and the 


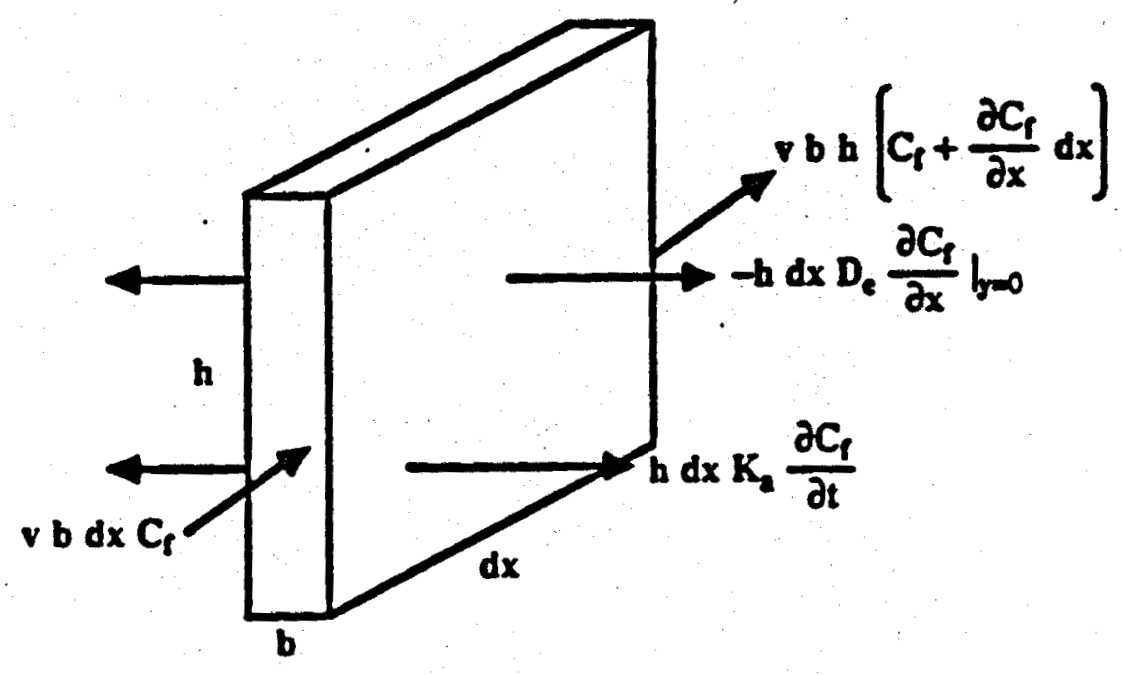

Figure 2.1: Fracture Flow

coupling of the matrix diffusion model to chromatographic analysis.

Derivation of the Governing Equations

The two governing equations are expressions of material balance. Figures 2.1 and 2.2 show the control volumes used to develop the fracture flow and matrix diffusion governing equations. The fracture and adjoining matrix are assumed to be homogeneous and isotropic. Convection, diffusion and adsorption occur within the system, and while longitudinal dispersion terms are not included, Taylor Dispersion is used to describe diffusion within the fracture itself (Taylor 1953). Taylor Dispersion holds that diffusion in the direction of flow is negligible in relation to the velocity of flow but is quick enough so that there is no concentration gradient across the small width of the fracture. While the effective diffusivity is used throughout this report, Neretnieks (1980) presents an excellent survey of the various diffusion constants used in other literature. Adsorption is modeled as linear, instantaneous reaction (mass adsorbed is proportional to the 


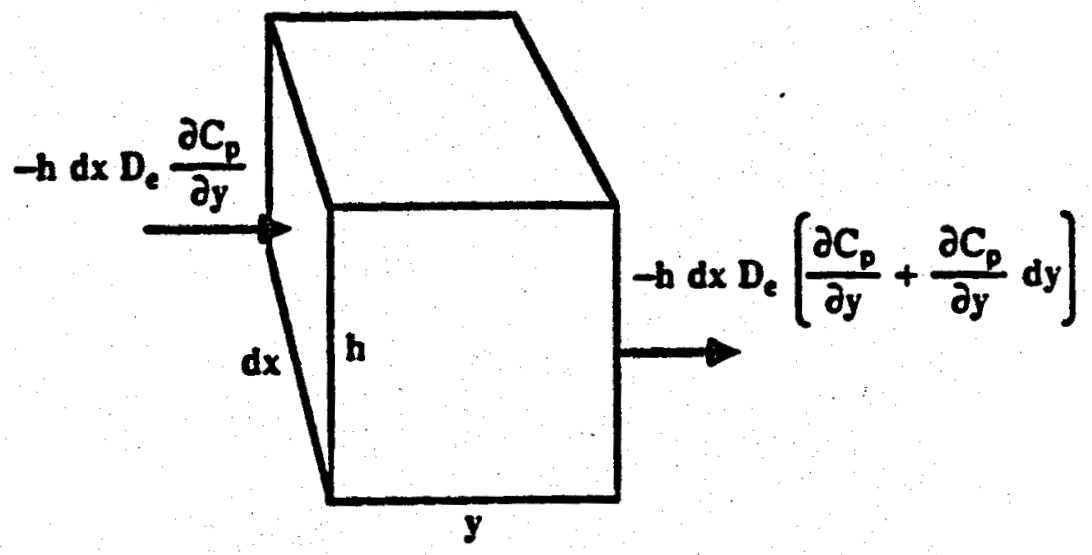

Figure 2.2: Matrix Diffusion

concentration).

Fracture Flow Governing Equation

The fracture flow equation describes the transport of a tracer through a fissure. Figure 2.1 depicts a fracture with a width, $b$, a height, $h$, and a differential length, $d x$. A tracer of concentration $C_{f}$ is transported through the fracture with a velocity, v. Fick's Law of diffusion describes the flux of tracer which flows through the fracture wall. Tracer is also lost from the fracture due to adsorption which is characterized as a linear function of concentration. $K_{a}$, the areal partition coefficient, models the equilibrium between the mass of tracer adsorbed onto the fracture wall and the concentration of the tracèr in the fracture.

Placing the terms shown in Figure 2.1 into a mass balance equation:

$$
\begin{aligned}
& \text { MassEntering - MassLeaving }=\text { MassAccumulating } \\
& v b h C_{f}-v b h\left(C_{f}+\frac{\partial C_{f}}{\partial x} d x\right)+\left.2 h d x D_{e} \frac{\partial C_{f}}{\partial y}\right|_{y=0}-2 h d x K_{a} \frac{\partial C_{f}}{\partial t}
\end{aligned}
$$




$$
=h b d x \frac{\partial C_{f}}{\partial t}
$$

Dividing both sides of the equation by $h b d x$ yields:

$$
-v \frac{\partial C_{f}}{\partial x}+\left.\frac{2}{b} D_{e} \frac{\partial C_{f}}{\partial y}\right|_{y=0}-\frac{2}{b} K_{a} \frac{\partial C_{f}}{\partial t}=\frac{\partial C_{f}}{\partial t}
$$

Note that $h$ dropped out of the equation.

Rearranging Equation 2.3:

$$
\left(1+\frac{2}{b} K_{a}\right) \frac{\partial C_{f}}{\partial t}=\left.\frac{2}{b} D_{e} \frac{\partial C_{f}}{\partial y}\right|_{y=0}-v \frac{\partial C_{f}}{\partial x}
$$

The retardation factor, $R_{D}$, is defined to be $\left(1+\frac{2}{b} K_{a}\right)$ (Freeze and Cherry 1979), hence:

$$
R_{D} \frac{\partial C_{f}}{\partial t}=\left.\frac{2}{b} D_{e} \frac{\partial C_{f}}{\partial y}\right|_{y=0}-v \frac{\partial C_{f}}{\partial x}
$$

This article will refer to both Equations 2.4 and 2.5 as the fracture flow governing equation.

Matrix Diffusion Governing Equation

The matrix diffusion equation describes the transport of tracer through the porous matrix. Figure 2.2 depicts an elemental volume representing the matrix. Fick's Second Law of Diffusion models the diffusion of tracer through the rock where $C_{p}$ denotes the concentration in the pores of the matrix (mass per volume of liquid). Mass accumulates in the volume due to adsorption onto the rock grains.

Placing the terms shown in Figure 2.2 into a mass balance equation:

$$
\begin{aligned}
& \text { MassEntering - MassLeaving = MassAccumulating } \\
& -(h d x) D_{e} \frac{\partial C_{p}}{\partial y}-\left[-(h d x) D_{c}\left(\frac{\partial C_{p}}{\partial y}+\frac{\partial^{2} C_{p}}{\partial y^{2}} d y\right)\right]=(h d x d y) \frac{\partial C_{m}}{\partial t}
\end{aligned}
$$


$C_{m}$ is the concentration within a given volume of rock (mass per volume of liquid and solid).

Dividing both sides of the equation by (hdx)dy yields:

$$
D_{e} \frac{\partial^{2} C_{p}}{\partial y^{2}}=\frac{\partial C_{m}}{\partial t}
$$

Again $h$ divides out of the equation. $h$ does not appear in either governing equation. The solution will not depend on the fracture height.

The mass in the matrix, $C_{m}$ (mass per bulk volume), is comprised of free solute in the pores, $C_{p}$ (mass per pore volume), and adsorbed mass on the grains $S$ (mass per bulk volume).

$$
C_{m}=\phi C_{p}+S
$$

For small concentrations a linear isotherm may be used to model the partitioning between the rock and fluid. $K_{v}$ (pore volume per bulk volume) is the volumetric partition coefficient. $K_{v}$ is related to $K_{\varepsilon}$ by the surface area to volume ratio of the rock.

$$
S=K_{v} C_{p}
$$

Substituting Equation 2.10 into Equation 2.9

$$
C_{m}=\left(\phi+K_{v}\right) C_{p}
$$

Equations 2.8 and 2.11 may be combined:

$$
\left(\phi+K_{v}\right) \frac{\partial C_{p}}{\partial t}=D_{c} \frac{\partial^{2} C_{p}}{\partial y^{2}}
$$

Some authors define $\left(\phi+K_{v}\right)$ as the volumetric equilibrium constant, $K_{d} \rho_{p}$ and utilize the apparent diffusivity, $D_{a}$ where $D_{a}=D_{e} / K_{d} \rho_{p}$ (Neretrieks 1980). Equation 2.13 is the matrix diffusion governing equation.

$$
\frac{\partial C_{p}}{\partial t}=\frac{D_{c}}{\phi+K_{v}} \frac{\partial^{2} C_{p}}{\partial y^{2}}
$$




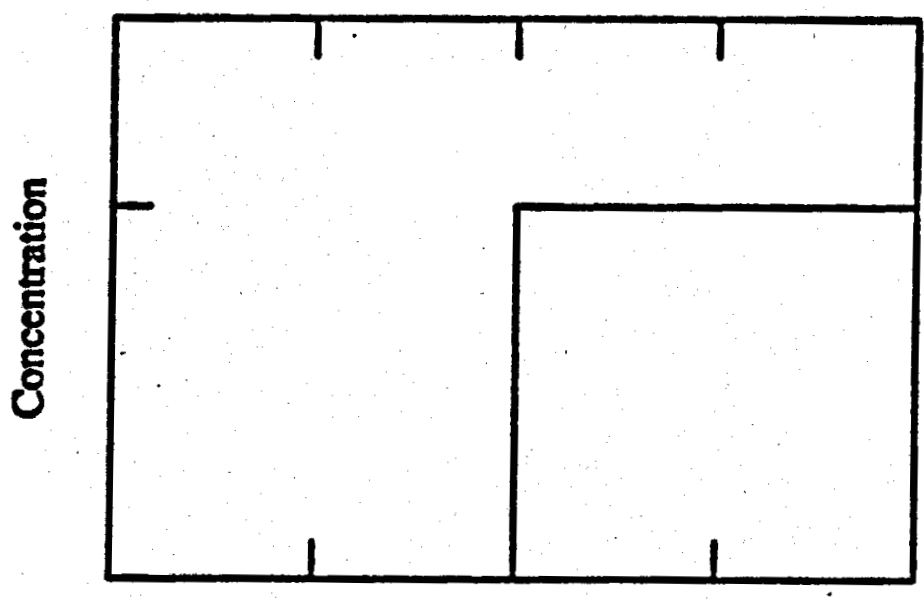

Time

Figure 2.3: Inlet Condition - Step Change

\section{Solution}

The governing equations may be solved for a step change at the inlet (Figure 2.3) where there is no tracer present prior to injection (Carslaw and Jaegar 1959). The equations were nondimensionalized by using six dimensionless groups. Concentration was nondimensionalized by dividing by the injection or reference concentration $\left(C_{D}=C / C_{0}\right)$. The lengths were divided by the distance between the inlet and outlet $\left(x_{D}=x / L\right.$ etc.). The retardation factor, $R_{D}$, is the same as previously defined. $K_{D}$ is the dimensionless partition coefficient where $K_{D}=\phi+K_{v}$.

Two groups were defined using an unknown time, $t^{*}$. $t^{*}$ defines the length of the fracture divided by the velocity of flow $(L / v)$. This is the time for a tracer to travel from an injector to a producer where there is no adsorption or diffusion. It is the breakthrough time for a nonsorbing tracer. The dimensionless time and dimensionless diffusivity are defined as 


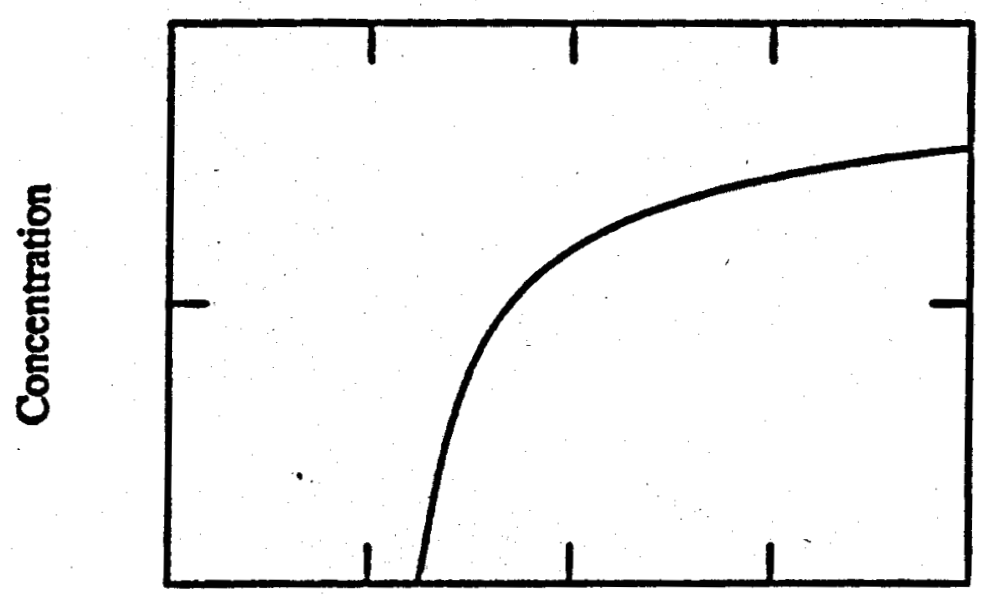

Time

Figure 2.4: Outlet Response to Step Change

follows: $t_{D}=t / t^{*} ; D_{D}=D_{e} t^{*} / L^{2}$

The solution to the step input is given in Equation 2.14. Notice that the retardation factor is buried with the dimensionless time inside a square root. The retardation factor does not affect the shape of the response. It simply translates the profile forward or backward in time. Also at the outlet or producing well, $x=L$ or $x_{D}=1$; therefore, the solution does not depend of the fracture length. Figure 2.4 shows the response to a step input.

$$
\begin{gathered}
C_{f D}=\operatorname{erfc}\left[\frac{x_{D}\left(D_{D} K_{D}\right)^{1 / 2}}{b_{D}\left(t_{D}-x_{D} R_{D}\right)^{1 / 2}}\right] ; \text { for } t_{D}>R_{D} \\
C_{J D}=0 ; \text { for } t_{D} \leq x_{D} R_{D}
\end{gathered}
$$

Fracture Aperture Equation

Most field tests do not usually involve simple step changes at the inlet. Instead, tracers are more commonly injected over a short period of time. When a tracer is injected into an injection well for a time, $\Delta t$ (Figure 2.5), 


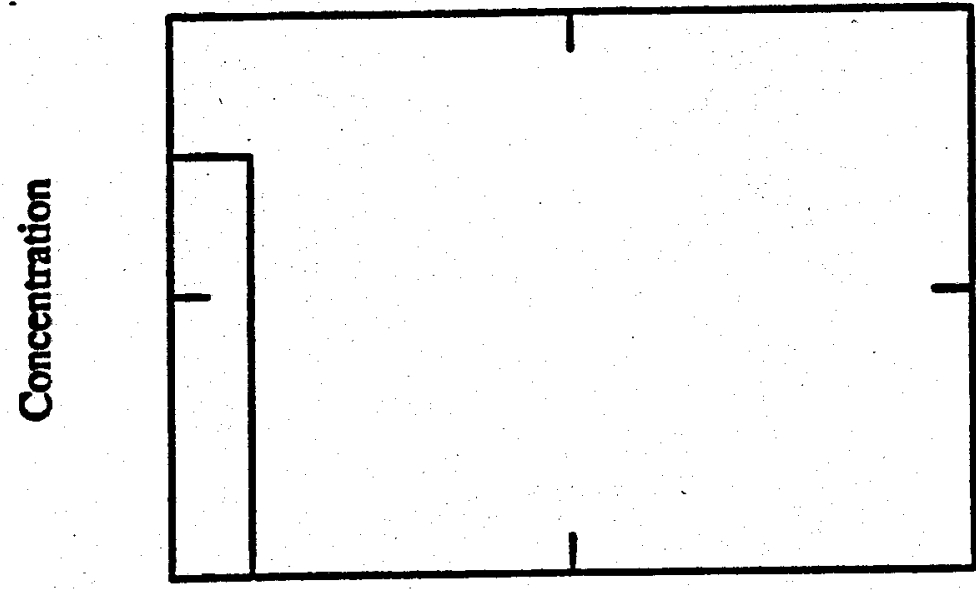

Time

Figure 2.5: Inlet Condition - Pulsed Injection

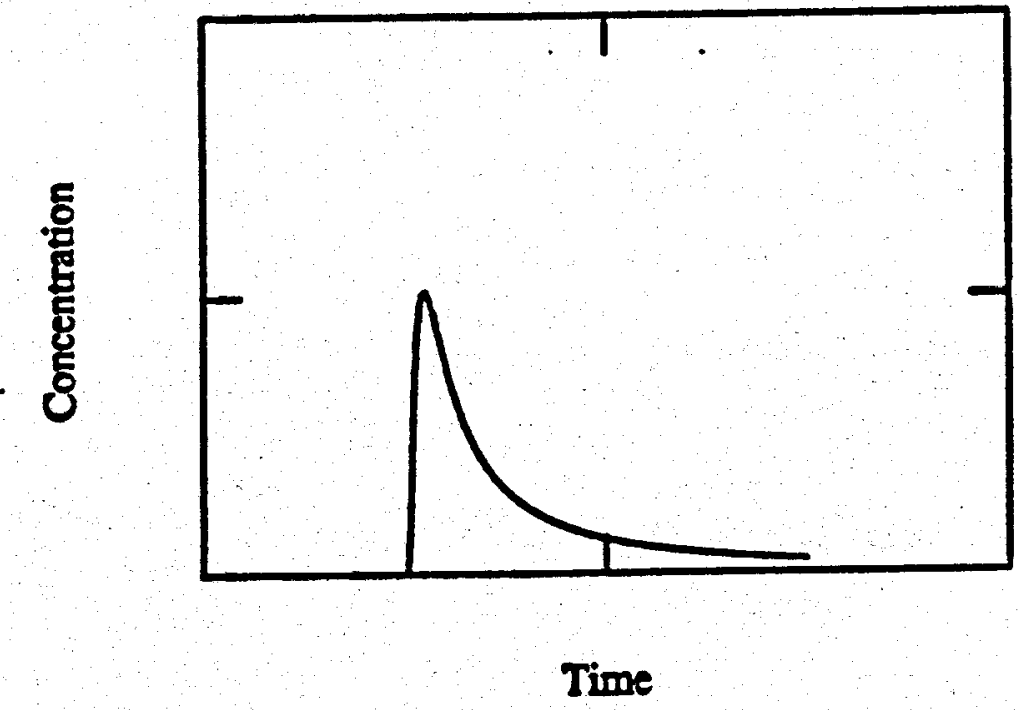

Figure 2.6: Outlet Response to Pulsed Injection 
the response will be as shown in Figure 2.6. This boundary condition can be handled by superposition of the step change solution (Equation 2.14). Equation 2.16 is the derivative of the solution to this input condition.

$$
\begin{gathered}
C_{f D}^{\prime}=A_{D}\left\{\frac{\exp \left[\frac{-D_{D} K_{D} x_{D}^{2}}{b_{D}^{2}\left(t_{D}-R_{D}\right)}\right]}{\left(t_{D}-x_{D} R_{D}\right)^{3 / 2}}-\frac{\exp \left[\frac{-D_{D} K_{D} x_{D}^{2}}{b_{D}\left(t_{D}-\Delta t_{D}-R_{D}\right)}\right]}{\left(t_{D}-\Delta t_{D}-x_{D} R_{D}\right)^{3 / 2}}\right\} \\
\text { for } t_{D}-\Delta t>x_{D} R_{D} \\
\text { where } A_{D}=\frac{x_{D}\left(D_{D} K_{D}\right)^{1 / 2}}{\sqrt{\pi} b_{D}\left(t_{D}-x_{D} R_{D}\right)^{3 / 2}}
\end{gathered}
$$

At the peak of the tracer recovery curve, the derivative of the concentration with respect to time is zero. By setting Equation 2.16 to zero and rearranging the result in dimensional form, one can derive the fracture aperture equation (Equation 2.17). Equation 2.17 relates the fracture aperture to $t^{*}$ and several other parameters. The other parameters can be determined through logs or laboratory tests on cores. The fracture aperture appears on both sides of the equation and an iterative solution is required.

Fracture Aperture Equation

$$
\begin{gathered}
b^{2}=\frac{D_{c}\left(\phi+K_{v}\right) t^{* 2} \Delta t}{\left(t_{p}-R_{D} t^{*}\right)\left(t_{p}-\Delta t-R_{D} t^{*}\right) \ln \left[1-\frac{\Delta t}{b_{p}-R_{D} t^{*}}\right]^{-3 / 2}} \\
\text { where } R_{D}=1+\frac{2}{b} K_{c}
\end{gathered}
$$

For no adsorption $\left(K_{\mathrm{a}}=K_{v}=0\right)$ :

$$
b^{2}=\frac{D_{e} \phi t^{2} \Delta t}{\left(t_{p}-t^{*}\right)\left(t_{p}-\Delta t-t^{*}\right) \ln \left[1-\frac{\Delta t}{t_{p}-R_{D} t^{*}}\right]^{-3 / 2}}
$$


For an instantaneous or spike injection, $\Delta t$ approaches zero. This is the normal boundary condition for injection of a radioactive tracer. Equation 2.19 is the fracture aperture equation for instantaneous injection. This expression provides a noniterative solution.

Fracture Aperture Equation - Instantaneous Injection

$$
b=\frac{K_{a} t^{*}+\sqrt{K_{a}^{2} t^{* 2}+6 D_{e}\left(\phi+K_{v}\right)\left(t_{p}-t^{*}\right) t^{* 2}}}{3\left(t_{p}-t^{*}\right)}
$$

For no adsorption $\left(K_{a}=K_{v}=0\right)$ :

$$
b=t^{*} \sqrt{\frac{2 D_{e} \phi}{3\left(t_{p}-t^{*}\right)}}
$$

Inferring values of $b$ from the fracture aperture equation requires the injection of either one nonsorbing or two dissimilar adsorbing tracers. Either method will allow estimation of $t^{*}$. This parameter appears in every form of the aperture equation and must be determined before the fracture width can be estimated. When a nonsorbing tracer is injected, the breakthrough time corresponds to $t^{*}$, and the aperture can be calculated in a straightforward manner. $t^{*}$ cannot be determined directly when dealing with a sorbing tracer because adsorption causes a retardation of the tracer movement. When one sorbing tracer is utilized, two unknowns, $b$ and $t^{*}$, appear in the one equation.' If two tracers with different affinities for adsorption are injected, one can write the fracture aperture equation twice - once for each tracer. With two equations, the two unknowns can both be calculated. Although the evaluation is simpler when using a nonsorbing tracer, it is important to note that truly nonsorbing tracers are uncommon. Skagius, Svedberg and Neretnieks (1982) have noted the adsorption of radioactive elements on minerals.

In many cases adsorption is the major cause for delaying the peak returns from $t^{*}$. Adsorption will dominate the slower diffusion process. Remember that surface adsorption will translate the entire profile while diffusion will simply separate the breakthrough and peak times. Figure 2.7 illustrates this point. Tracer 1 is a nonsorbing tracer; therefore, the breakthrough time and $t^{*}$ are identical. Diffusion causes the small difference 


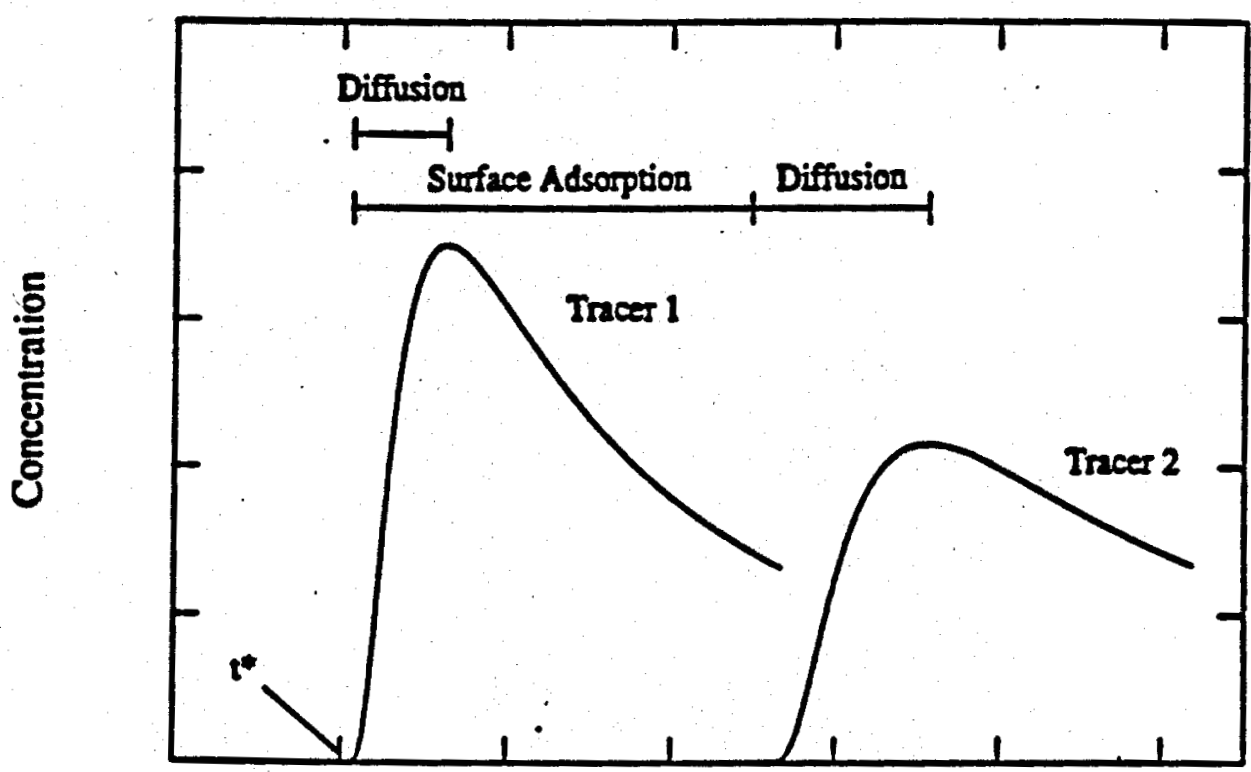

Time

Figure 2.7: Chromatographic Effects of Diffusion and Adsorption

between $t^{*}$ and $t_{p}$. Tracer 2 is an adsorbing tracer in which adsorptive effects cause a translation. This translation is the major reason that $t^{*}$ and the peak time are different. Notice the relatively small diffusional effects. For the case where adsorptive effects dominate:

$$
t_{p}-\Delta t-R_{D} t^{*} \approx 0
$$

Substituting for $R_{D}$ and solving for $b$ yields the:

Approximate Fracture Aperture Equation

$$
b \approx \frac{2 K_{a} t^{*}}{t_{p}-\Delta t-t^{*}}
$$

Note the great dependence on $K_{a}$. This is unfortunate because $K_{a}$ is the most difficult parameter to measure.

Analysis of Synthetic Tracer Tests

Synthetic tracer tests were generated using the matrix diffusion model. They are analyzed in this section to illustrate the utility of the fracture 


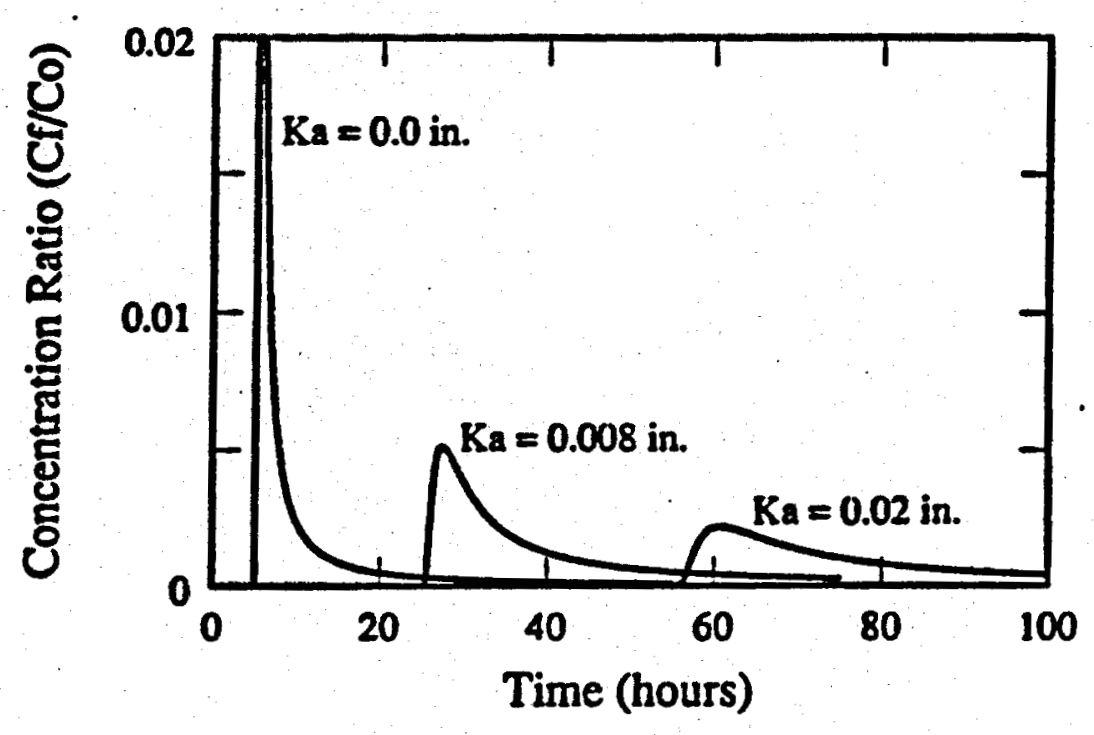

Figure 2.8: Synthetic Tracer Breakthrough Curves

aperture equation.

Figure 2.8 shows breakthrough tracer returns for three tracers. The flow is through a fracture of 0.004 inches in width. Tracer 1 is nonsorbing. Tracer 2 has an areal partition coefficient of twice the aperture. Tracer 3 's areal partition coefficient is five times the width. The input data are summarized in Tables 2.1 and 2.2 .

It is a simple matter to analyze the nonsorbing tracer test. The breakthrough and peak times are determined from the graph. Along with these parameters, the diffusivity, porosity and injection time are plugged into Equation 2.18. Note that the breakthrough time for the nonsorbing tracer is equal to $t^{*}(5 \mathrm{hr}=1000 \mathrm{ft} / 200 \mathrm{ft} / \mathrm{hr})$.

When analyzing adsorbing tracers, two tracers are necessary. Figure 2.9 presents a cross plot of $t^{*}$ vs. fracture aperture for tracers 2 and 3 . The approximate equation (2.22) was used to generate these curves. There is little separation between the two curves; highlighting the sensitivity of the solution to $K_{a}$. The lines cross at $5.15 \mathrm{hrs}$ and 0.0037 in. while the input parameters were 5 hours and 0.004 in. If the iterative equation were 
Table 2.1: Synthetic Tracer Test - Formation Data

\begin{tabular}{|c|c|c|}
\hline \multicolumn{3}{|c|}{ Fracture Data } \\
\hline$b$ & 0.004 & in. \\
\hline $\boldsymbol{x}$ & 1000 & $f t$ \\
\hline $\boldsymbol{h}$ & 100 & $f t$ \\
\hline$v$ & 200 & $f t / h r$ \\
\hline \multicolumn{3}{|c|}{ Matrix Data } \\
\hline $\begin{array}{l}\phi \\
D_{e}\end{array}$ & $\begin{array}{r}2 \\
.10^{-6}\end{array}$ & $\begin{array}{l}\% \\
f t^{2} / h r\end{array}$ \\
\hline
\end{tabular}

Table 2.2: Synthetic Tracer Test - Injection Data

\begin{tabular}{|c|c|c|}
\hline Tracer 1 & $\begin{array}{c}\text { Tracer Data } \\
\text { Tracer } 2\end{array}$ & Tracer 3 \\
\hline $\begin{array}{ll}K_{v} & 0.000 \\
K_{c} & 0.000 \text { in }\end{array}$ & $\begin{array}{ll}K_{v} & 0.500 \\
K_{c} & 0.008 \text { in. } \\
\end{array}$ & $\begin{array}{ll}K_{v} & 1.000 \\
K_{e} & 0.020 \text { in. } \\
\end{array}$ \\
\hline$\Delta$ & 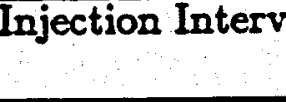 & $5 \mathrm{~min}$ \\
\hline
\end{tabular}




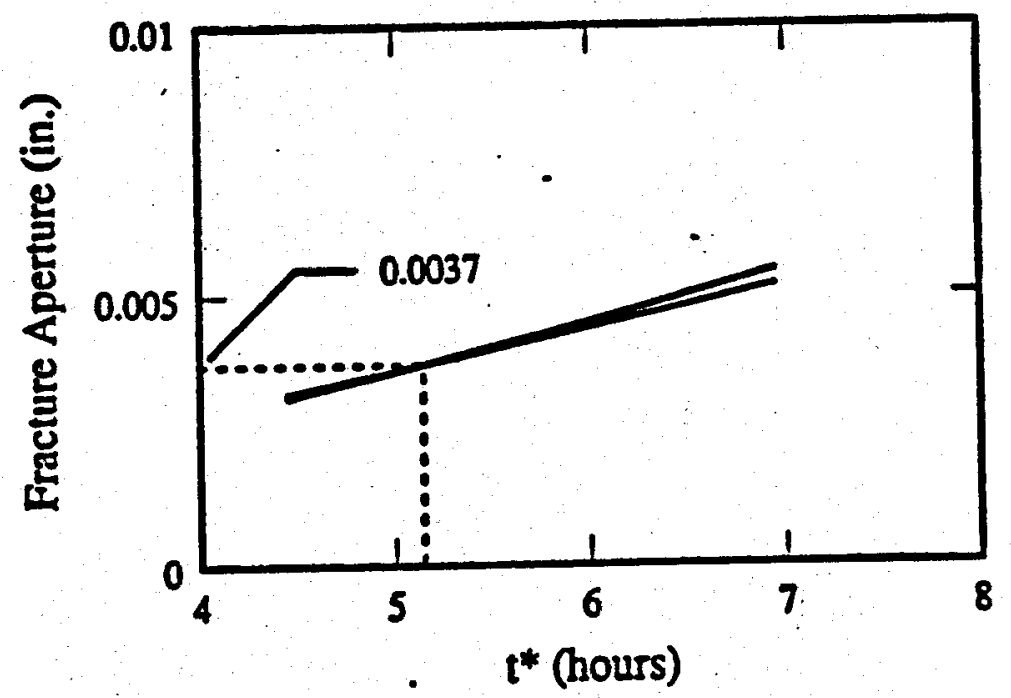

Figure 2.9: Fracture Aperture Cross Plot - Approximate Equation

used (Figure 2.10), the solution would be exact (excluding round off error). Figure 2.11 compares the two solutions.

\subsection{ANALYSIS OF WAIRAKEI FIELD DATA}

This section describes the analysis of tracer tests from the Wai- rakei Geothermal Field in New Zealand. The results compare favorably to those of an investigation by Jensen (1983). While he also used the matrix diffusion model, his analysis was done using a nonlinear regression program.

\section{Geology}

Wairakei is one of New Zealand's larger geothermal resources. The field lies in a large thermal area, 200 miles north of Wellington on the North Island. The primary production comes from the interface between the Waiora and Wairakei formations, and the primarily flow paths are through fissures. 


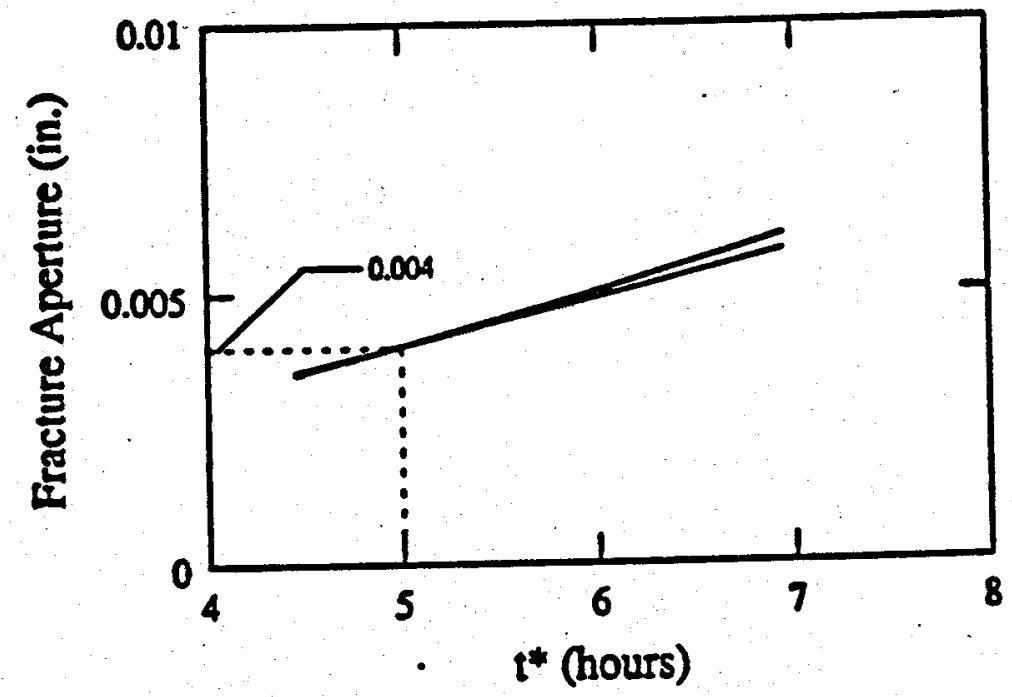

Figure 2.10: Fracture Aperture Cross Plot - Iterative Solution

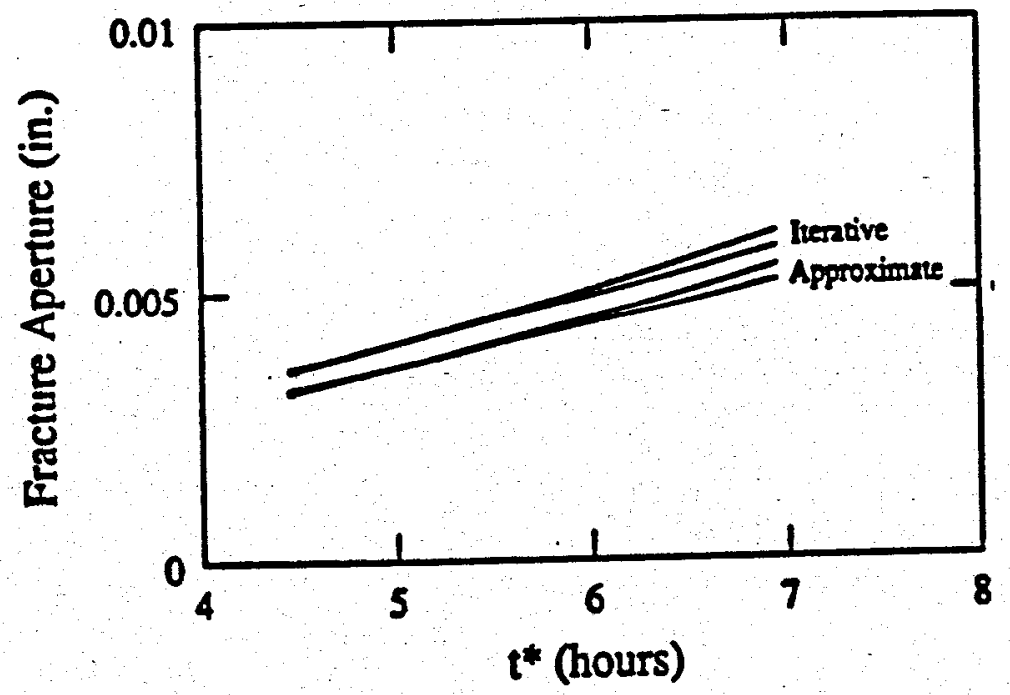

Figure 2.11: Approximate vs. Iterative Solution 


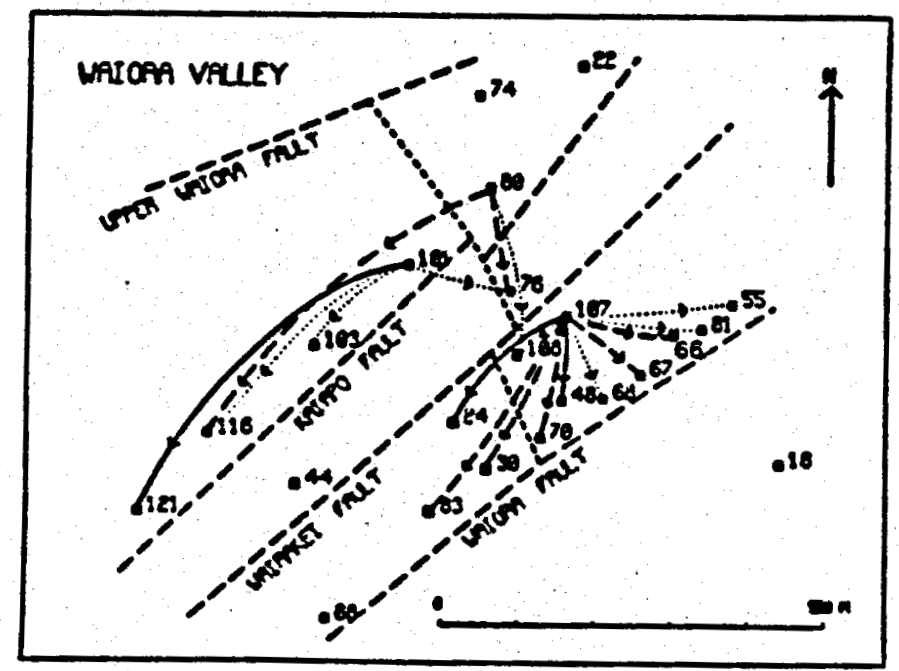

Figure 2.12: Well Locations in Wairakei Field (from McCabe at al, 1983)

The major faults, the Kaiapo, Wairakei and Waiora, all strike northeast to southwest and are intersected by minor faults inside the productive area (Figure 2.12). Jensen (1983) provides a fairly complete synopsis of the geology. For a more complete description see Grindley (1965).

Tracer Tests

Two tracer injection tests are analyzed in this report. They were performed by the Institute of Nuclear Sciences, Department of Scientific and Industrial Research, New Zealand. In March 1979, 155 GBq of iodine-131 were injected into well WK107 at 1096 feet (Figure 2.12). A vial containing the tracer was shattered inside a surface by-pass connection thus producing an instantaneous injection (McCabe, Barry and Manning 1983). Radioactivity was subsequently seen in ten wells. Later in June, $165 \mathrm{GBq}$ were injected into well WK101 at 1312 feet, and sensors detected the iodide in twelve wells. Some wells did not show significant response and were not 

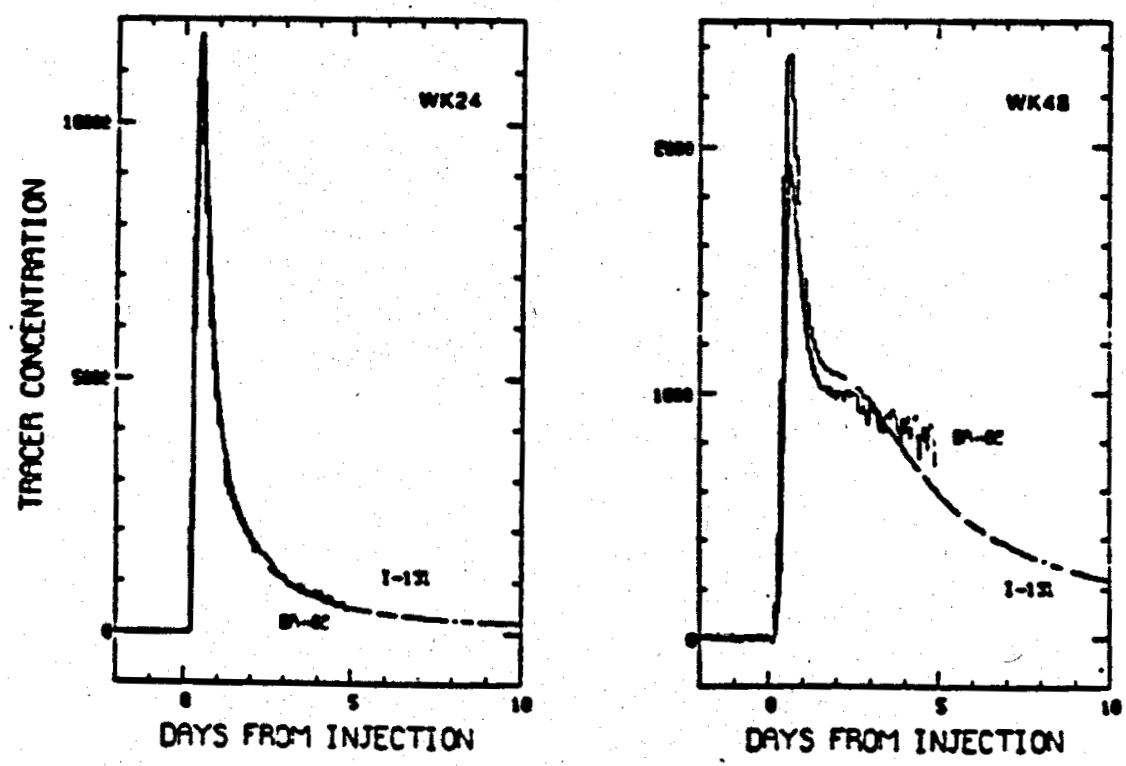

Figure 2.13: Chromatographic Movement of Iodide and Bromide - Wairakei (from McCabe et al. 1983)

analyzed by Jensen (1983).

Analysis and Comparison

The data could only be analyzed if an adsorption isotherm were assumed. A literature survey produced no publications regarding the sorption of iodide on the minerals found in the Wairakei field although the chromatographic performance of jodide vs. bromide was tested in Wairakei (Figure 2.13). There was no difference in the arrival times or shapes of the breakthrough curves (McCabe 1983). The responses may have been alike either because neither tracer adsorbed, or both adsorbed similarly. The similarity is probable since both iodide and bromide are halogens. A factor which tended to reduce the affinity of iodide for the rock was its electrical potential. Anions have little attraction for identically charged formations; however, they are known to adsorb onto the crystal edges of otherwise negatively charged clays (Gray and Darley 1980). In the absence of better information the best assumption was that iodide does not adsorb.

Equation 2.20, valid for a nonsorbing tracer, was used to analyze the 
data. Only four parameters were needed: porosity, diffusivity, breakthrough time and time to peak concentration. The two times were found by inspecting the concentration vs. time plots, and the porosity was estimated by logs and core analysis. The diffusivity could have been inferred from the porosity (Perkins and Johnston 1963) or measured directly. To facilitate comparison with Jensen's calculations, this analysis assumed a porosity of $1 \%$ and a diffusivity of $4.32 \cdot 10^{-6} \mathrm{ft}^{2} /$ day $\left(5 \cdot 10^{-7} \mathrm{~cm}^{2} / \mathrm{s}\right)$.

Equation 2.20 repeated:

$$
b=t^{*} \sqrt{\frac{2 D_{e} \phi}{3\left(t_{p}-t^{*}\right)}}
$$

From Perkins and Johnston (1963):

$$
D_{e}=\frac{D_{0}}{F \phi}
$$

For a carbonate or consolidated formation:

$$
F=\frac{1}{\phi^{2}}
$$

For a sandstone:

$$
D_{e}=\frac{0.62}{\phi^{2.15}}
$$

The analysis of WK68 was typical and proceeded as follows. A plot similar to Figure 2.14 was inspected. The breakthrough and peak times were determined visually, in this case 2.3 and 11.09 days. The fracture aperture of 0.0043 in. was then calculated using Equation 2.20.

Jensen used a nonlinear regression technique. Three parameters were needed to fit the data. $\alpha$ determined the shape of the curve. 1/ $\beta$ was equal to the breakthrough time, and the third factor was a simple scaling coefficient. The time to peak concentration was not determined explicitly but could be found by manipulating Jensen's equations. For the WK68 Jensen computed a breakthrough time of 2.92 days and a peak time of 11.09 days. The resulting fracture aperture was 0.0068 in., approximately 


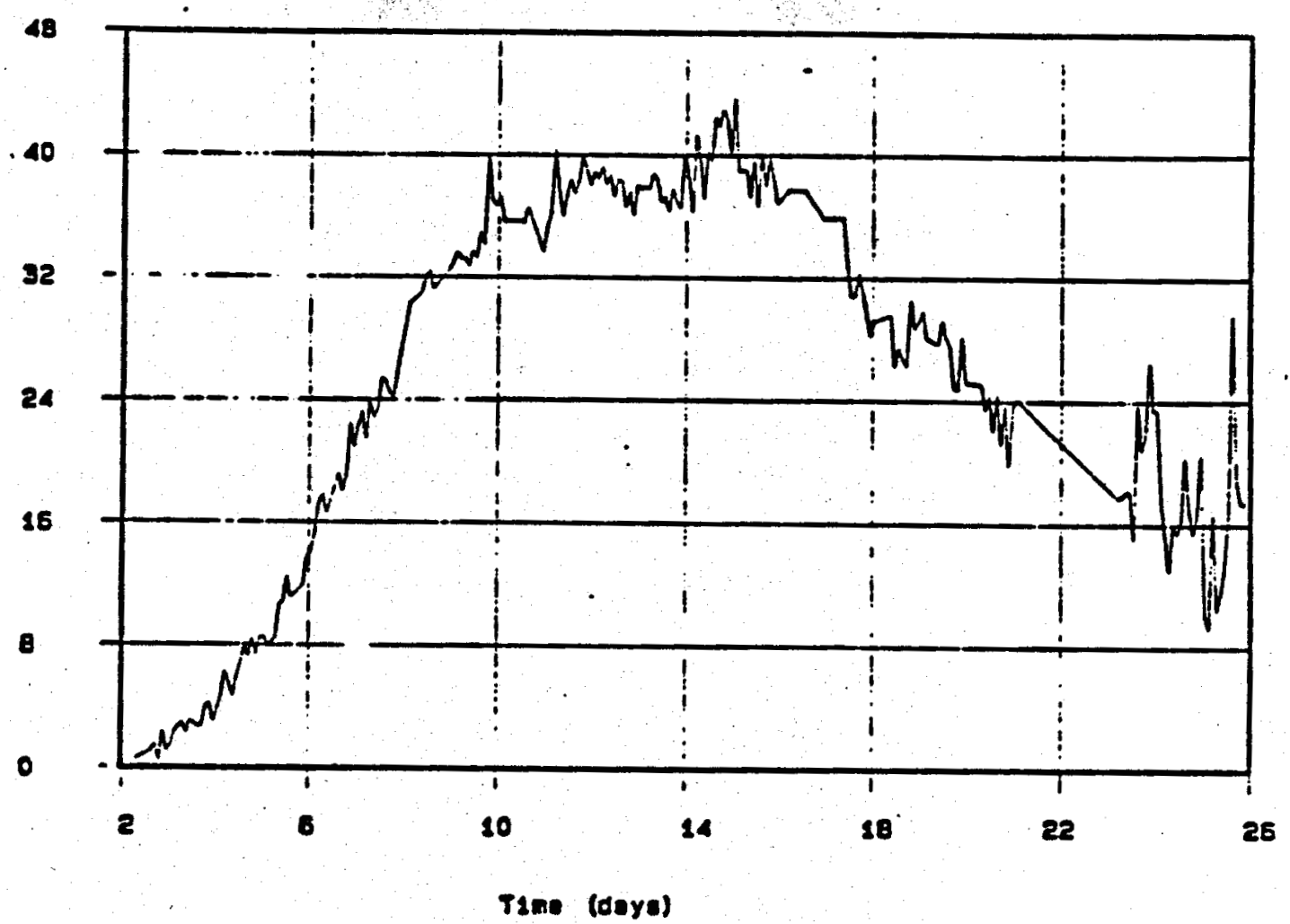

Figure 2.14: WK68 Tracer Breakthrough Curve - from WK107

$50 \%$ greater than the width estimated visually. Since both methods were based on the matrix diffusion model, identical picks for the times yielded identical aperture estimates.

Table 2.3 presents the results of the visual and regression methods. In three cases Jensen found a better match using a double fracture model. Visual analysis of this kind was impossible. When only the ten single fracture fit curves were compared, the estimates of fracture aperture agreed fairly well. Figure 2.15 shows a plot of width found from the regression and visual procedures. The divergence between the two methods lie within the definitions of accuracy and precision. The average absolute value of the difference between the procedures was $40 \%$ (low precision): If the differences are summed while maintaining the positive and negative signs, the average of the discrepancies is only $0.9 \%$ (high accuracy).

Figures 2.16 and 2.17 attempt to discern the reasons for the discrepancies. The regression method computed both longer breakthrough and peak times. Since the difference between the two times is important, the relative overestimation was not the cause of the divergence. The difference 
Table 2.3: Estimation of Fracture Aperture - Regression and Visual Methods

\begin{tabular}{|c|c|c|c|c|c|c|c|c|c|}
\hline Inj. & Prod. & & $\operatorname{Reg}$ & ession & & & Visual & & \\
\hline Well & Well & $\alpha$ & $\begin{array}{l}1 / \beta \\
\text { days }\end{array}$ & $\begin{array}{c}t_{p} \\
\text { days }\end{array}$ & $\begin{array}{l}b \\
\text { in. }\end{array}$ & $\begin{array}{l}t^{*} \\
\text { days }\end{array}$ & $\begin{array}{c}t_{p} \\
\text { days }\end{array}$ & $\begin{array}{c}b \\
\text { in. }\end{array}$ & $\begin{array}{l}\Delta b \\
\%\end{array}$ \\
\hline 107 & WK24 & 1.25 & 0.23 & 0.47 & 0.0031 & 0.22 & 0.51 & 0.0027 & -14 \\
\hline & WK30 & 1.37 & 4.37 & 9.83 & 0.013 & 3.7 & 9.3 & 0.010 & -16 \\
\hline & & 1.27 & 3.22 & 6.67 & 0.012 & & & & -7 \\
\hline & WK48 & 1.39 & 0.29 & 0.67 & 0.0032 & 0.26 & 0.70 & 0.0026 & -17 \\
\hline & & 1.67 & 1.04 & 2.97 & 0.0050 & & & & -48 \\
\hline & WK55 & 2.58 & 2.67 & 14.51 & 0.0052 & 3.3 & 15.7 & 0.0063 & -23 \\
\hline & WK67 & 2.74 & 1.65 & 9.89 & 0.0038 & 0.46 & 15.3 & 0.0008 & -80 \\
\hline & WK68 & 2.05 & 2.92 & 11.09 & 0.0068 & 2.3 & 14.8 & 0.0043 & -35 \\
\hline & WK70 & 2.48 & 2.03 & 10.39 & 0.0047 & 2.8 & 9.9 & 0.0070 & +50 \\
\hline & WK81 & 1.54 & 3.66 & 9.41 & 0.010 & 4.0 & 8.4 & 0.013 & +23 \\
\hline & WK83 & 2.17 & 2.55 & 10.53 & 0.0060 & 3.7 & 9.7 & 0.010 & +73 \\
\hline & WK108 & 1.69 & 6.78 & 19.62 & 0.013 & 10.7 & 25.6 & 0.019 & +47 \\
\hline 101 & WK103 & 3.44 & 0.62 & 5.49 & 0.0019 & 0.54 & 5.1 & 0.0017 & -10 \\
\hline & WK116 & 3.84 & 0.63 & 6.79 & 0.0017 & 2.4 & 7.0 & 0.0075 & +342 \\
\hline & & 0.92 & 4.79 & 7.35 & 0.019 & & & & -61 \\
\hline & WK121 & 0.92 & 1.45 & 2.26 & 0.011 & 1.1 & 2.5 & 0.0062 & -41 \\
\hline
\end{tabular}




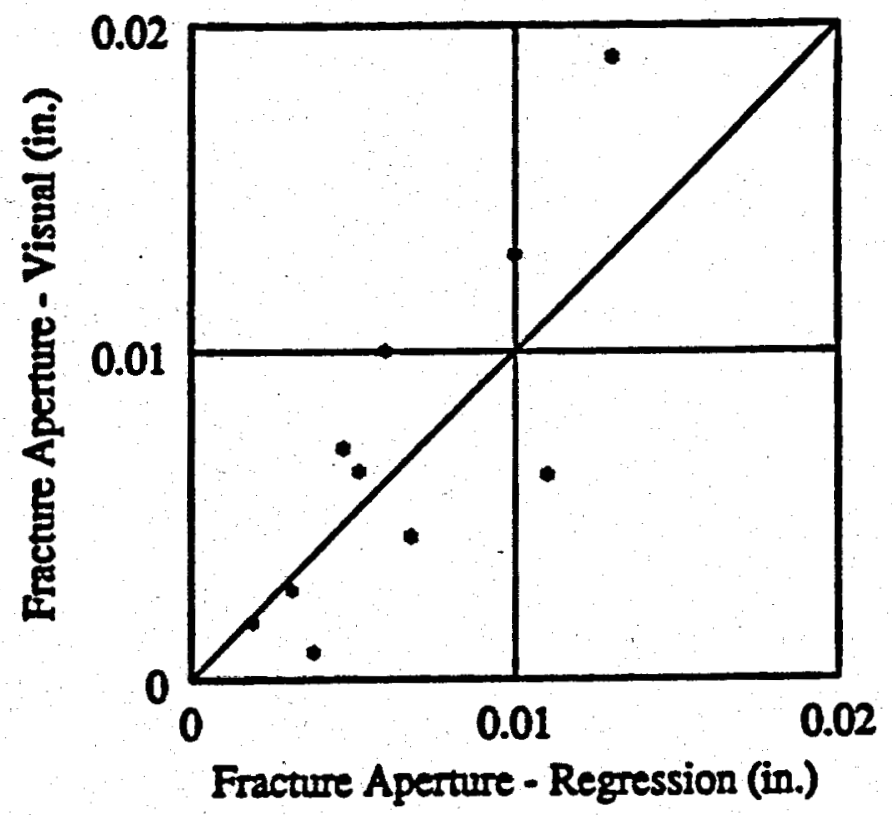

Figure 2.15: Fracture Aperture - Visual vs. Regression Method

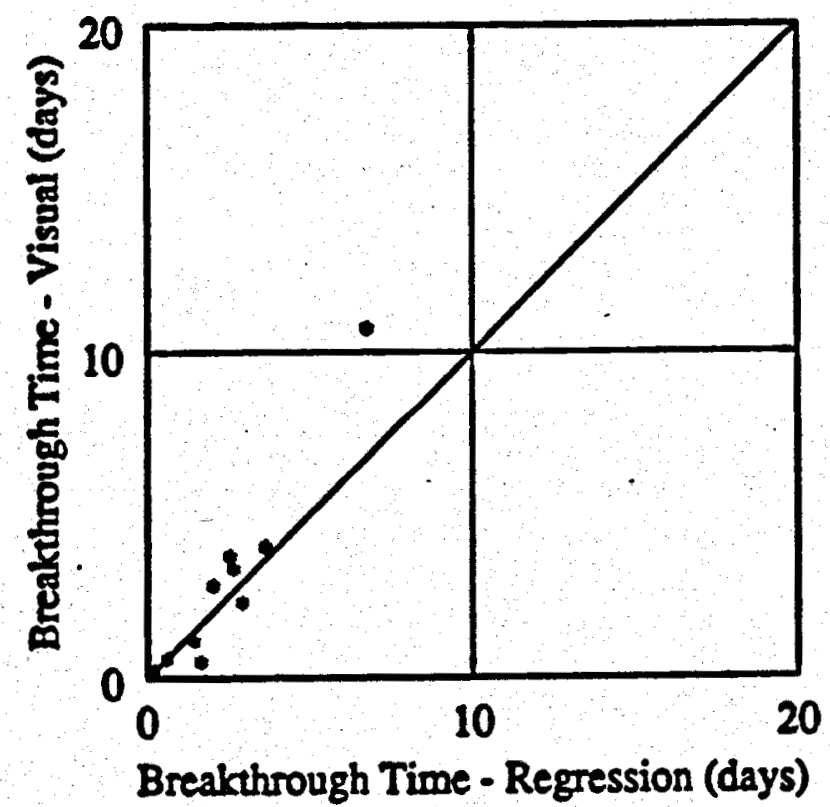

Figure 2.16: Breakthrough Time - Visual vs. Regression Method 


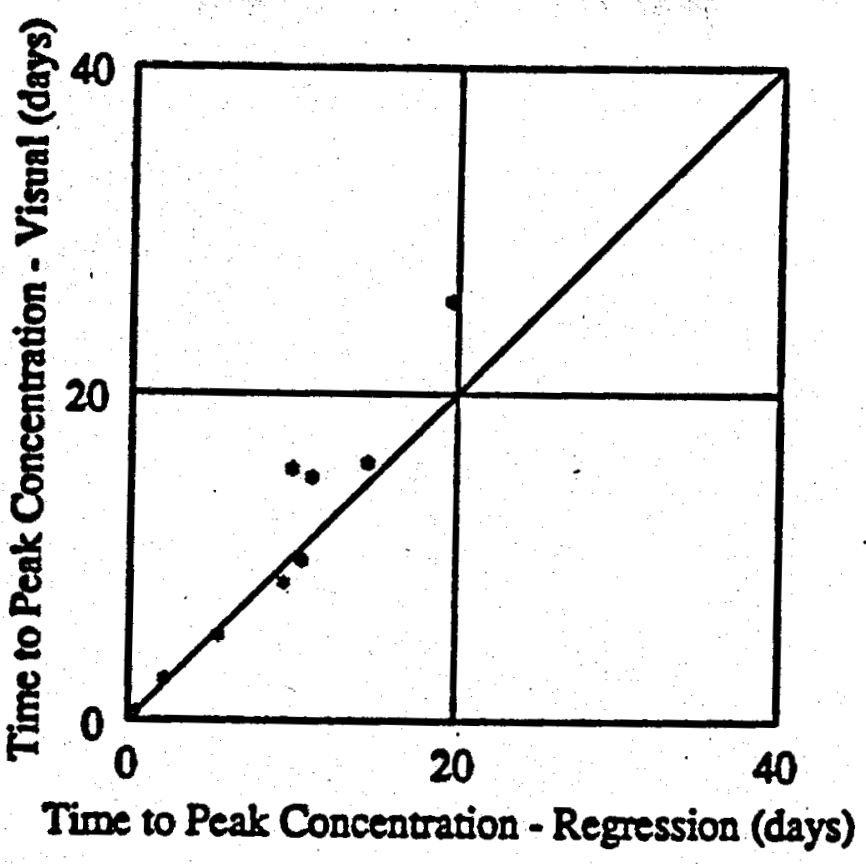

Figure 2.17: Peak Concentration Time - Visual vs. Regression Method

was simply a random variation. Perhaps with practice, an engineer could fine tune his judgement.

The visual estimation was probably more accurate in at least one case. In his analysis of the WK116 well (Figure 2.18), Jensen incorporated data from 0 to 2.4 days. These points included random background radiation. Their inclusion may be the origin of the double fracture fit.

\subsection{CONCLUSIONS}

1. Adsorption is a significant factor in the flow of tracers through through fractured rock. This process can occur in two places, on the fissure wall and in the matrix. The effect of areal adsorption is to translate the entire breakthrough curve in time while adsorption within the rock pores tends to increase dispersion. In the case of a pulsed injection, volumetric adsorption increases the time between breakthrough and the peak concentration. Estimating fracture aperture from tracer 


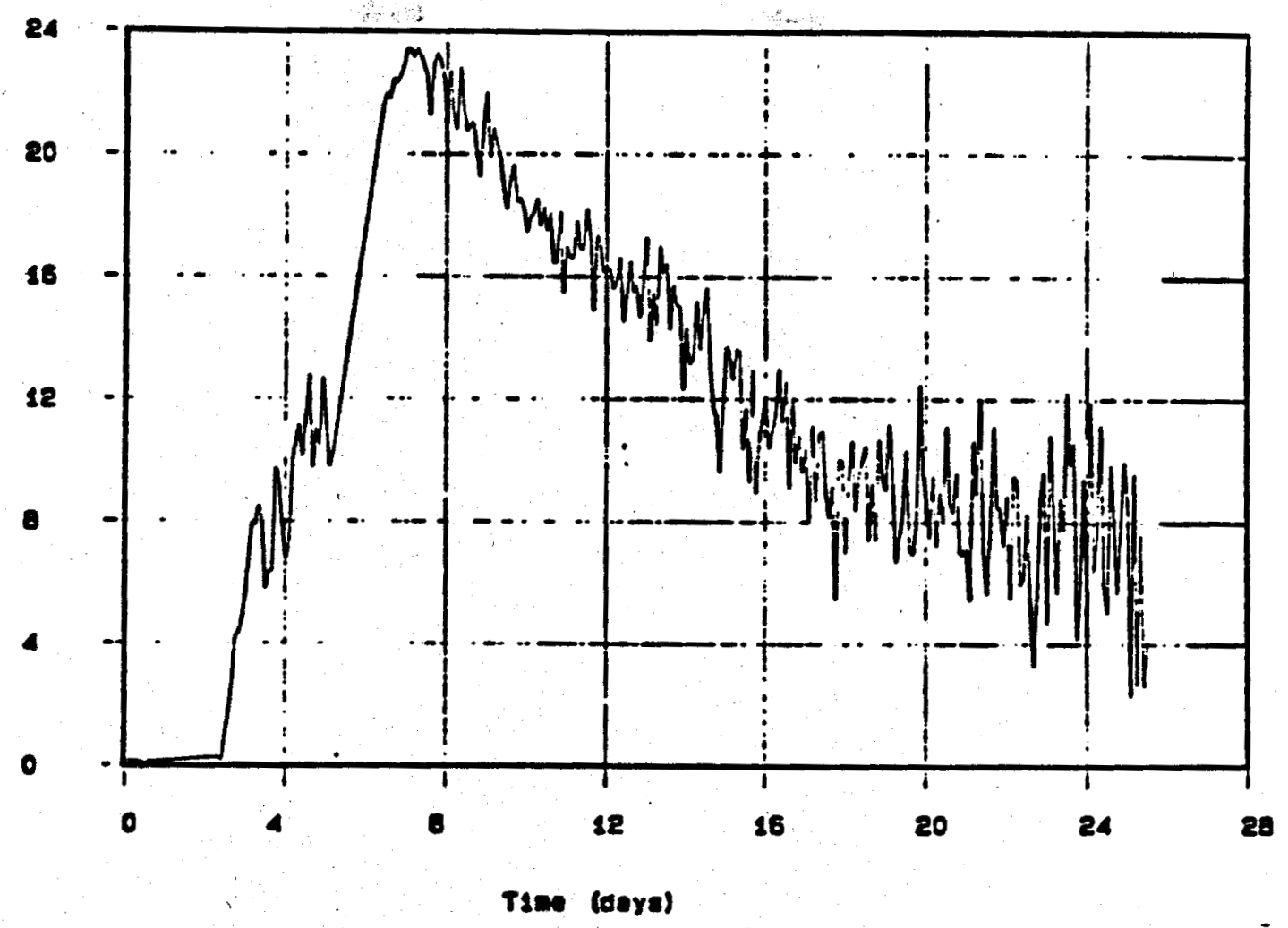

Figure 2.18: WK116 Tracer Breakthrough Curve - from WK101

tests requires an accurate estimation of the partition coefficients.

2. The fracture aperture may be determined by visually examining tracer breakthrough curves. Accurate estimation of fissure width requires good appraisals of the times to breakthrough and peak concentration. Choosing the correct values of $R_{D} t^{*}$ and $t_{p}$ is not always easy especially when analyzing radioactive tracer data. 


\subsection{NOMENCLATURE}

$A_{D}$ dimensionless coefficient

$b$ fracture aperture, $[L]$

$b_{D} \quad$ dimensionless fracture aperture

$C$ concentration (mass/volume), $\left[\mathrm{m} / \mathrm{L}^{3}\right]$

$C_{D}$ dimensionless concentration $\left(C / C_{0}\right)$

$C_{f}$ concentration in fracture (mass/liq. vol.), $\left[\mathrm{m} / L^{3}\right]$

$C_{f D}$ dimensionless concentration in fracture $\left(C_{f} / C_{0}\right)$

$C_{m} \quad$ concentration in matrix (mass/vol. of rock), $\left[\mathrm{m} / \mathrm{L}^{3}\right]$

$C_{p}$ concentration in pores (mass/liq. vol.), $\left[\mathrm{m} / L^{3}\right]$

$C_{p D}$ dimensionless concentration in pores $\left(C_{p} / C_{0}\right)$

$C_{0} \quad$ injection or reference concentration, $\left[\mathrm{m} / \mathrm{L}^{3}\right]$

$C_{f D}$ dimensionless fracture concentration $\left(C_{f} / C_{0}\right)$

$C_{1}, C_{2}$ constants in conversion of voltage to concentration

$D_{a} \quad$ apparent diffusivity $\left(D_{e} /\left(\phi+K_{v}\right)\right),\left[L^{2} / t\right]$

$D_{D} \quad$ dimensionless diffusivity $\left(D_{e} t^{*} / L^{2}\right)$

$D_{c} \quad$ effective diffusivity, $\left[L^{2} / t\right]$

$D_{0}$ molecular diffusivity in water, $\left[L^{2} / t\right]$

F formation factor

$h$ fracture height, $[L]$

$K_{a} \quad$ areal partition coefficient, $[L]$

$K_{v} \quad$ volumetric partition coefficient, (pore vol/bulk vol)

$K_{d} \rho_{p} \quad$ volumetric equilibrium constant $\left(\phi+K_{v}\right)$

$K_{D} \quad$ dimensionless partition coefficient $\left(\phi+K_{v}\right)$

$L$ fracture length, $[L]$

$R_{D} \quad$ retardation factor $\left(1+f K_{a}\right)$

$S \quad$ concentration of mass adsorbed on rock

(mass/rock volume), $\left[\mathrm{m} / \mathrm{L}^{3}\right]$

$t$ time, $[t]$

$t_{D} \quad$ dimensionless time $\left(t / t^{*}\right)$

$t_{p} \quad$ time from injection to peak concentration, $[t]$

$t^{*} \quad t-\operatorname{star}(L / v),[t]$

$\Delta t \quad$ injection time, $[t]$

$\Delta t_{D}$ dimensionless injection time $\left(\Delta t / t^{*}\right)$

$v$ velocity of flow in fracture, $[L / t]$

$x \quad$ length along fracture axis, $[L]$ 
$x_{D}$ dimensionless fracture length

$y$ distance normal to fracture axis into matrix, $[L]$

$y_{D}$ dimensionless distance into the matrix

$\phi$ porosity

\subsection{REFERENCES}

1. Carslaw, H. S. and Jaeger, J. C., 1959. Conduction of Heat in Solids. Oxford University Press, New York, Second Edition, p. 396.

2. DiPippo, Ronald, 1980. Geothermal Energy as a Source of Energy. U. S. Government Printing Office, First Edition, p. 210.

3. Freeze, R. Allan and Cherry, John A., 1979. Groundwater. PrenticeHall Inc., Englewood Cliffs, New Jersey, p. 402-413.

4. Gray, George R. and Darley, H. C. H., 1980. Composition and Properties of Oil Well Drilling Fluids. Gulf Publishing Company, Houston, Texas, Fourth Edition.

5. Grindley, G. W., 1965. The Geology, Structure and Exploitation of the Wairakei Geothermal, Taupo, New Zealand. New Zealand Geological Society Bulletin, v. 75, p. 104.

6. Jensen, Clair L., 1965. Matrix Diffusion and Its Effects on the Modeling of Tracer Returns from the Fractured Geothermal Reservior at Wairakei, New Zealand. Technical Report SGP-TR-71, Stanford Geothermal Program.

7. McCabe, W. J., Barry, B. J. and Manning M. R., 1983. Radioactive Tracers in Geothermal Underground Water Flow Studies. Geothermics, v. 12 , p. $83-110$.

8. Neretnieks, Ivars, 1980. Diffusion in the Rock Matrix: An Important Factor in Radionuclide Retardation? Jour. of Geophys. Res. v. 85 p. $4379-4397$.

9. Perkins, T. K. and Johnston, O. C., 1963. A Review of Diffusion and Dispersion in Porous Media. Soc. of Pet. Eng. Jour., March. 
10. Skagius, K., Svedberg, G. and Neretnieks, I., 1982. A Study of Strontium and Cesium Sorption on Granite. Nuclear Technology, v. 59, p. 302-313.

11. Taylor, Sir Geoffrey F.R.S., 1953. Dispersion of Soluble Matter in Solvent Flowing Slowly through a Tube. Proceedings of the Royal Society of London, v. 219 p. 186-203. 


\section{Chapter 3}

\section{Analysis of Tracer and Thermal Transients During Reinjection}

Ibrahim Kocabas and Roland N. Horne

\subsection{INTRODUCTION}

In geothermal reservoir engineering, many field experiences have shown that the reinjected water may move through the fractures to the production zones in a very short time. This rapid migration of the thermal front can be avoided if we can identify the fast flow channels prior to the reinjection. The analysis of the return profiles from tracer tests is the tool most commonly used to identify these channels and to estimate the fracture aperture (which is the most important parameter governing the advancement of thermal front). A quantitative analysis of the tracer return profiles can be accomplished by matching the solutions to equations of mathematical models to the return profiles.

Today there are three commonly used mathematical models to represent the tracer transport through fractures. The first of these models, the convection-dispersion (CD) model, assumes a purely dispersive flow in the fracture. It considers the fracture as a plane between two parallel plates. 
The spreading of the tracer is attributed to the combination of the transverse molecular diffusion and convective dispersion along the flow direction. The governing differential equation of the convection-dispersion model is given by

$$
\frac{\partial C}{\partial t}+u \frac{\partial C}{\partial x}-D \frac{\partial^{2} C}{\partial x^{2}}=0
$$

The second model is called the matrix diffusion (MD) model, and represents a system consisting of a fracture, in which the tracer fluid is mobile, located in a porous matrix, in which the reservoir fluid is virtually immobile. It neglects the longitudinal dispersion and assumes that the diffusion of the tracer from the fracture into the adjacent porous matrix is the mechanism spreading the tracer along the transport region. In the matrix, the diffusive transport is assumed to occur perpendicular to the flow direction in the fracture.

Two coupled one-dimensional equations are used to represent the tracer transport. The equations are coupled by using the continuity of the fluxes and the concentrations across the fracture-matrix interface. The equation of the tracer transport in the fracture is given by

$$
\frac{\partial C}{\partial t}+u \frac{\partial C}{\partial x}+q=0
$$

and the equation of the transport in the matrix is

$$
D_{a} \frac{\partial^{2} C_{m}}{\partial y^{2}}+=\frac{\partial C_{m}}{\partial t}
$$

The concentrations at the fracture-matrix interface are equated as

$$
C=C_{m} \text { at } y=0
$$

and the continuity of fluxes at the interface gives $q$ in Eq. 3.3 as

$$
q=-\left.\frac{2 D_{e}}{b} \frac{\partial C_{r}}{\partial y}\right|_{y=0}
$$
by

The effective and apparent diffusion coefficients, $D_{e}$ and $D_{a}$, are related 


$$
D_{e}=\phi D_{c}
$$

The third model, called the Advonin (AD) model, takes into account both the longitudinal dispersion and the diffusion into the matrix. It was developed by Advonin to study the temperature distribution in an oil layer during the injection of a hot incompressible fluid. The model has been widely used to study transport of tracers. As in the matrix diffusion model, the tracer transport in the system is represented by two coupled one-dimensional equations. The fracture transport equation is given by

$$
\frac{\partial C}{\partial t}+u \frac{\partial C}{\partial x}-D \frac{\partial^{2} C}{\partial x^{2}}+q=0
$$

The matrix transport equation and the equations describing the continuity of the concentrations and the fluxes are given by Eq. 3.3 , Eq. 3.4 and Eq. 3.5 respectively.

A variable of a system is defined as a characteristic which may be measured and which assumes different numerical values when measured at different times and points. In tracer test analysis, the use of either of two different concentration variables, namely resident or flux concentrations, has been equally common.

In the development of the mathematical equations, the resident concentration (the amount of the tracer per unit volume of the system at a given instant) has usually been taken as the variable of the system. In experiments, on the other hand, the flux concentration (the ratio of the tracer flux to the volumetric flux) has been the most commonly measured quantity. As a result, the return profiles have most commonly been plotted by using the flux concentration as the output variable. These two concentrations differ whenever the system is dispersive and there is a concentration gradient. Consequently, whenever a dispersive model is used for interpreting the return profiles, failure to distinguish between these two concentration variables leads to the use of solutions derived for initial and boundary conditions inconsistent with the actual conditions of the experiment(tracer test).

In order to investigate the properties of geothermal reservoirs, the geother$\mathrm{mal}$ industry has conducted interwell tracer tests both with and without 
recirculation, as well as injection-backflow tracer tests. In the following sections we present the analysis of the return profiles of these different types of tracer tests, conducted in two different fields.

\subsection{INTERWELL TESTS - NO RECIRCU- LATION}

In an interwell tracer test without recirculation we introduce the tracer into the system at the injection well and observe it at the production well(s). The tracer can be transported either by the natural gradient of the flow system or by the pressure gradient caused by injection and production. We can use several different methods to inject and detect the tracer. In order to use the correct theoretical solution to interpret the return profiles, we need to determine which of the two concentration variables (flux or resident) corresponds to these injection and detection modes.

We have obtained three data sets from the tracer tests conducted in the Wairakei geothermal feld. The tracer was introduced into the system at the bottom of the release wells and allowed to be transported by the gradient created by the production wells. In this type of tracer test, the flux mode is the appropriate model for both injection and detection. For an instantaneous injection in the flux mode, the solutions and the pertinent boundary conditions are given in Table 3.1.

A multiple parameter nonlinear regression program, VARPRO developed at Stanford's Computer Science Department, was used to match the solutions to the return profiles. To select the best of the three models representing our system, we applied three criteria commonly used in model evaluations. First, the model match should have a small residual. Second, it should capture distinctive features of the profiles, such as breakthrough time, peak arrival time and total recovery. Finally, values of the parameters recovered through curve fitting must be physically possible and consistent with the basic assumptions used in the development of the model.

One of the most sought-after parameters is the fracture aperture, which can be estimated by two methods. If the condition of the Taylor dispersion theory, namely 


\begin{tabular}{|c|c|c|c|}
\hline Model & IC \& BC & Parameters & Solution \\
\hline$C D$ & $\begin{array}{c}C(x, 0)=0 \\
C(0, t)=\frac{m}{Q} \delta(t) \\
\lim _{x \rightarrow \infty} C(x, t)=0\end{array}$ & $\begin{array}{l}\alpha_{1}=\frac{1}{P_{c}}=\frac{D}{u L} \\
\alpha_{2}=\frac{1}{t_{u 1}}=\frac{u}{L}\end{array}$ & $C=\frac{m}{Q \sqrt{4 \pi \alpha_{1} \alpha_{2} t^{3}}} \exp ^{-\left(1-\alpha_{2} t\right)^{2} /\left(4 \alpha_{1} \alpha_{2} t\right)}$ \\
\hline MD & $\begin{array}{c}C(x, 0)=C_{m}(x, y, 0)=0 \\
C(0, t)=\frac{m}{Q} \delta(t) \\
C(x, t)=C_{m}(x, 0, t) \\
\lim _{y \rightarrow \infty} C_{m}(x, y, t)=0\end{array}$ & $\begin{array}{l}\alpha_{1}=\frac{1}{\sqrt{t_{d}}}=\frac{\sqrt{D_{d}}}{b} \\
\alpha_{2}=\frac{1}{t_{1}}=\frac{u}{l}\end{array}$ & $C=\frac{m H\left(t-\alpha_{2}\right) \alpha_{1} \alpha_{2}}{Q \sqrt{\pi\left(t-\alpha_{2}\right)^{3}}} \exp ^{-\left(\alpha_{1} \alpha_{2}\right)^{2} /\left(t-\alpha_{2}\right)}$ \\
\hline$A D$ & $\begin{array}{c}C(x, 0)=C_{m}(x, y, 0)=0 \\
C(0, t)=\frac{m}{Q} \delta(t) \\
\lim _{x \rightarrow \infty} C(x, t)=0 \\
C(x, t)=C_{m}(x, o, t) \\
\lim _{y \rightarrow \infty} C_{m}(x, y, t)=0\end{array}$ & $\begin{array}{c}\alpha_{1}=\frac{1}{P_{6}}=\frac{D}{u L} \\
\alpha_{2}=\frac{1}{t_{10}}=\frac{u}{L} \\
\alpha_{3}=\frac{1}{\sqrt{t_{2}}}=\frac{\alpha \sqrt{D_{2}}}{b}\end{array}$ & $C=\frac{m}{Q} \exp ^{1 /\left(2 \alpha_{1}\right)} \exp ^{-\sqrt{1 /\left(4 \alpha_{1}^{2}\right)+\left(\alpha+2 \alpha_{3} \sqrt{n}\right) /\left(\alpha_{1} \alpha_{2}\right)}}$ \\
\hline
\end{tabular}

Table 3.1: Solutions to Models of Non-recirculating Flow 


$$
\frac{L}{u} \gg \frac{0.5 b^{2}}{D_{m}}
$$

is satisfied it can be estimated by

$$
b=0.067345 \sqrt{\frac{t_{w}}{P_{e}}}
$$

for $C D$ and $A D$ models. Notice that $t_{w}$, the mean arrival time for water, is equal neither to the breakthrough time nor to the peak arrival time for these two models. It is, however, virtually equal to the breakthrough time of the MD model. The fracture aperture can also be estimated by

$$
b=\frac{2.93410^{-3} \phi}{\sqrt{t_{d}}}
$$

for $M D$ and $A D$ models.

The ability of each of the model solutions to match the field data are shown in Figures 3.1-3.5. For all the profiles analyzed here the residual error of the fit is of the same order for all three models. However, models differed in capturing the breakthrough and peak arrival times. Also, the values of the recovered parameters differed greatly. Fig. 3:1 shows the return profile at the well WK108 and the optimum fits for the models. In the return profile, considering the distance, $230 \mathrm{~m}$, between the injection well and the well WK108, we see that the breakthrough and peak arrival times are very small, indicating a fast flow path. The concentrations of the tail section are very close to the peak concentration which indicates a strong matrix diffusion effect. The dominant matrix diffusion effect becomes obvious since the matrix diffusion model captures the breaktrough and the peak arrival times as accurately as the Advonin model.

For CD and AD models, the estimated fracture apertures based on theTaylor dispersion theory are quite large and do not satisfy Eq. 3.8. However, for $\mathrm{MD}$ and $\mathrm{AD}$ model matches, the apertures estimated by using Eq. 3.10 are consistent with the observations since a fast flow and a strong matrix diffusion are likely to occur in a narrow fracture.

Fig. 3.2 shows the return profile at the well WK116 and the effectiveness of the models in matching the observed data. The profile has the characteristics of a fast flow and strong matrix diffusion, however in this case they are less pronounced compared to the characteristics of WK108. While CD 
and MD models have slight differences with the observed values, the AD model shows an excellent agreement with the data. However, the values of the parameters estimated in the regression differed significantly. For example, $t_{v}$, a parameter common to all of the three models is estimated as only 2.5 days for the MD model, 5.8 days for the AD model and 13.8 days for the CD model. The fracture apertures cannot be estimated by using Eq. 3.9 , because Eq. 3.10 is not satisfied. The MD model yields a smaller fracture aperture and a higher flow speed than the AD model.

Fig. 3.3 shows the return profile at the well WK76 matched by the CD model. The magnitude of the residual was similar to that of the earlier matches. The model yielded a relatively small flow velocity and a high dispersion, yet the fracture aperture estimated from Eq. 3.9 did not satisfy Eq. 3.10. We could not match MD and AD models to this set of data.

The existence of the double peak suggests that the injection and the observation well may be connected by two paths. The two path solution to the CD model gave a much better match, Fig. 3.4, but neither of the two path solutions to the MD and $A D$ models matched the profile.

During the iterations of the regression for a single path solution to the AD model, the Peclet number estimate consistently drifted towards infinity. A two path solution was also unsuccessful, but this time either the Peclet number estimate for the second path drifted towards infinity or ill-conditioning occured. We have concluded that the ill-conditioning occured because the flow rates of the two paths were almost the same. In this case, in the regression procedure, we need use one transfer function with six variables - three for each path.

As for the drifting of the Peclet number estimate for the second path towards infinity, it could be simply that the dispersion in the second path is negligible. Therefore, we matched the first peak by the AD model. Then the profile for this first path was subtracted from the observed profile and the resultant profile was matched by the MD model. The performances of the one transfer function for the AD model and for this combined AD-MD model are shown in Fig. 3.5. 


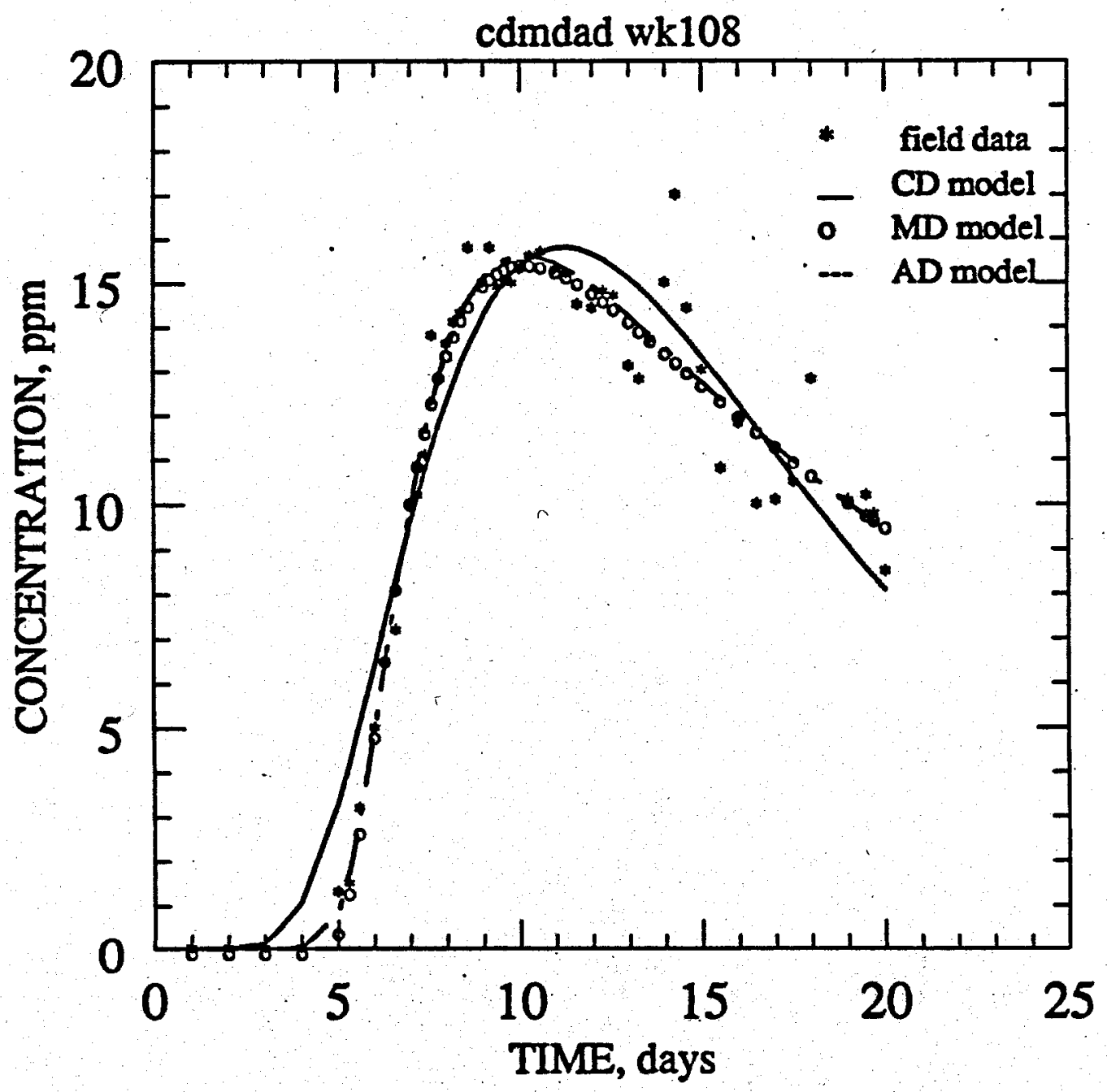

Figure 3.1: Model Fits to the Profile at WK108 


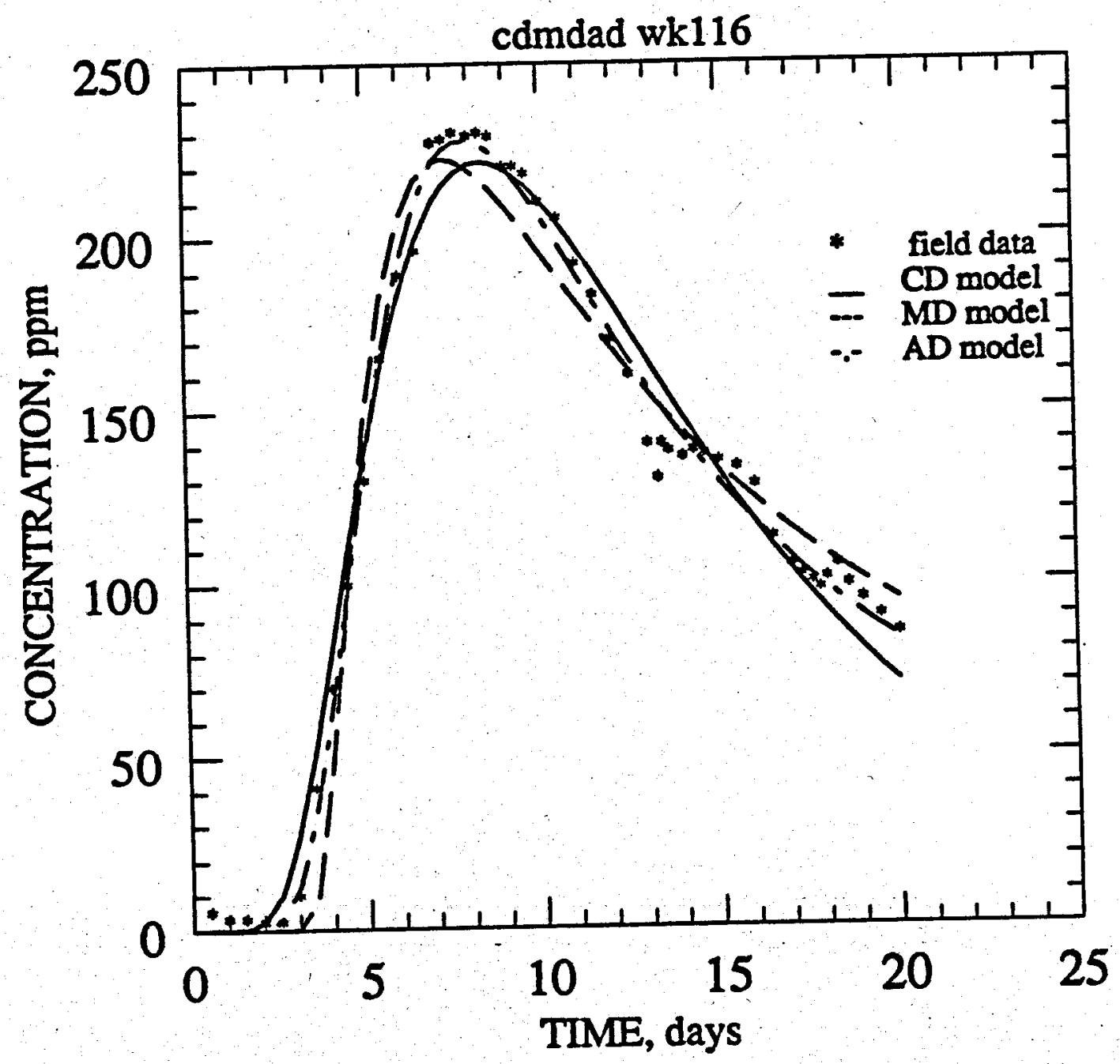

Figure 3.2: Model Fits to the Profile at WK116 


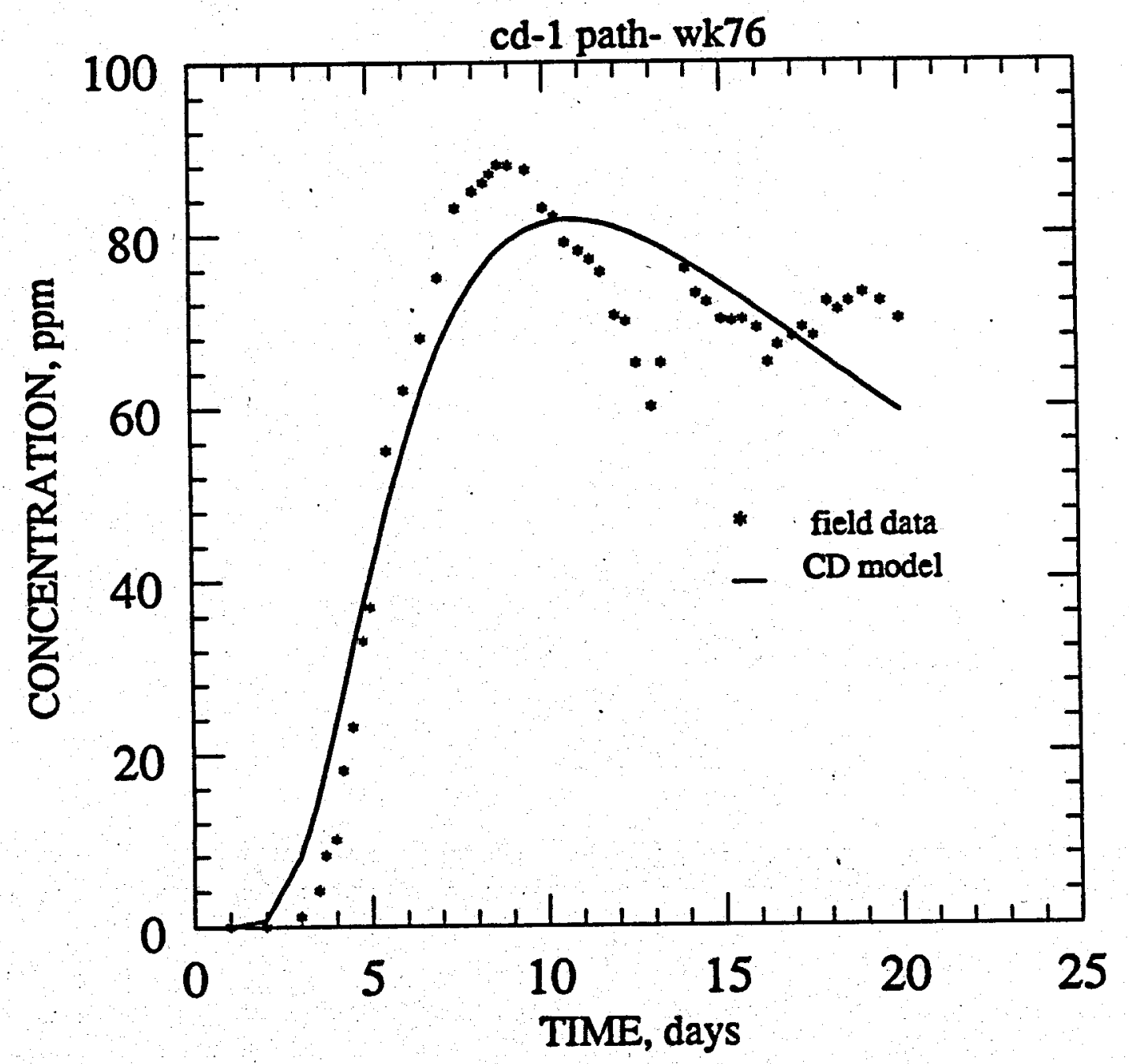

Figure 3.3: 1-Path CD Model's Fit to the Profile at WK76 


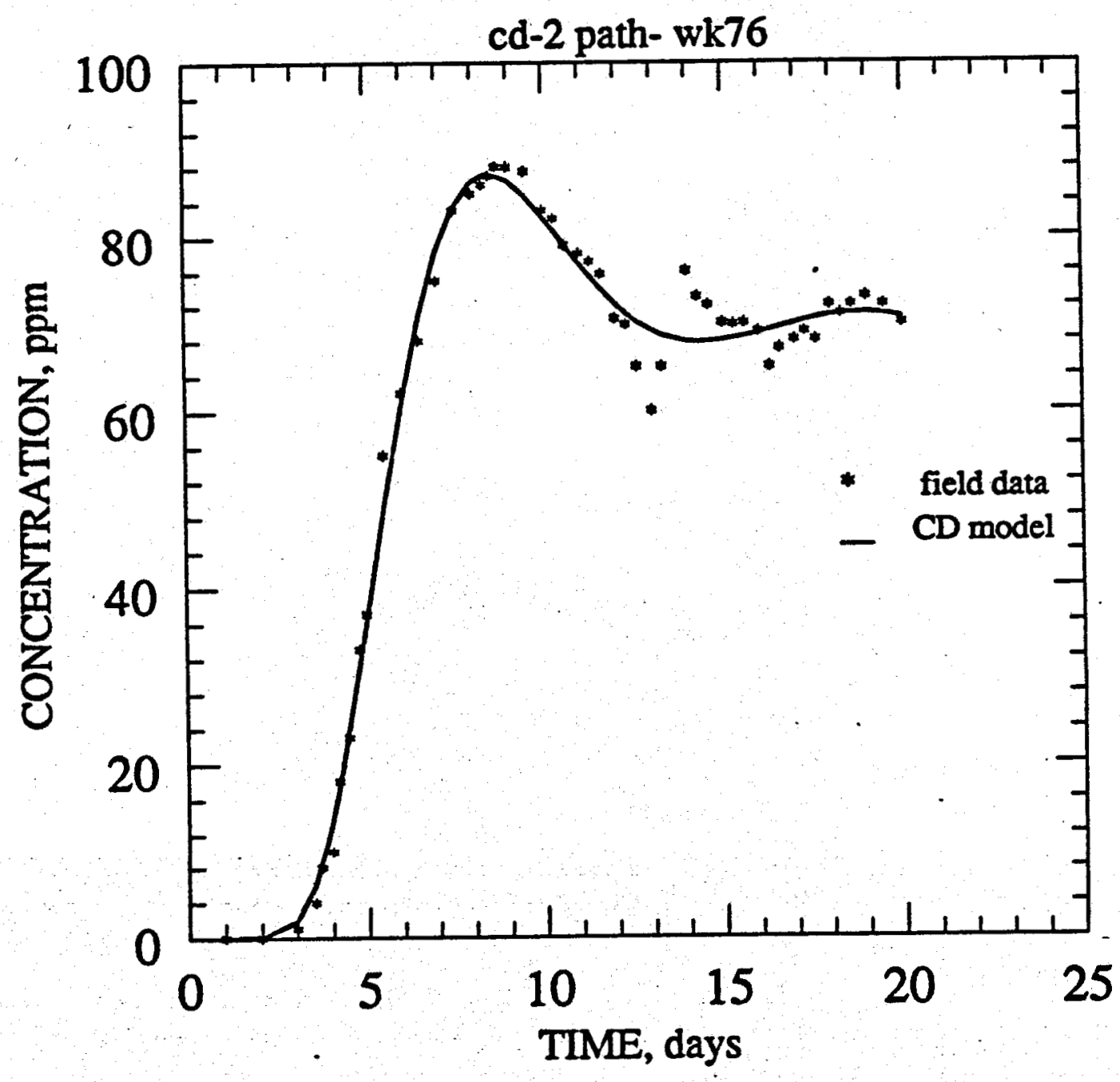

Figure 3.4: 2-Path CD Model's Fit to the Profile at WK76 


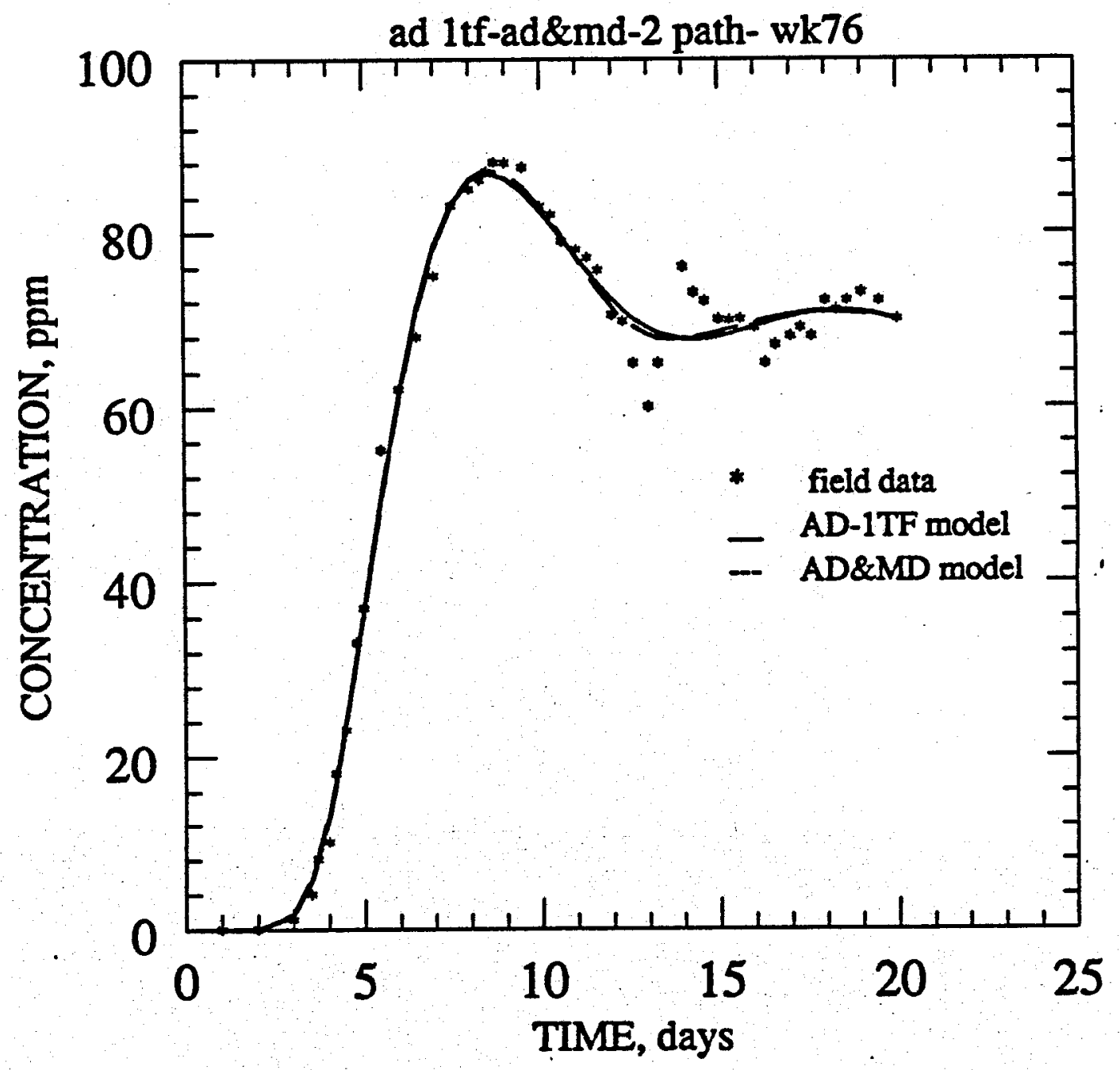

Figure 3.5: 2-Path AD and AD-MD Model's Fits to the Profile at WK76 


\subsection{INTERWELL TESTS WITH RECIRCU- LATION}

In an interwell tracer test with recirculation, the reservoir fluid is withdrawn from a production well and reinjected at an equal rate into the reservoir at the injection well. The procedure continues until the system reaches steady state. Then the tracer is introduced into the flowing stream at the injection well. While the recirculation continues the concentration history of the fluid samples taken at the production well is analyzed to determine the systems parameters. The techniques used to inject and detect the tracer correspond to injection and detection in flux concentration mode.

Interwell tracer tests with recirculation are useful to estimate the volume of the fluid recirculating in the system. Another advantage of this type of test is that it enables larger distances and areas to be investigated.

The previously developed methods of analysis do not account for the recirculation effects. Therefore the previous methods were only applicable in homogeneous layers with low dispersivity and wells situated at sufficient distance to minimize the recirculation effects. The solutions we have developed, however, not only specifically include the recirculation, but also use the effects of the recirculation to determine the dominant mechanism of tracer spreading along the flow path. The solutions were developed for instantaneous injection and detection in concentration flux mode. The solutions with the pertinent initial and boundary conditions are given in Table 3.2. Note that the solutions were derived by utilizing a semi-infinite domain. Several researchers have discussed the validity of the approach and they all prefer this flux-flux, semi-infinite medium solutions over the finite medium solutions. The semi-infinite and finite medium solutions give the same numerical values for all values of the parameters, but the latter is more complicated.

We have matched these solutions to the return profile of a test conducted in Broadlands, New Zealand. In this test, it was known that recirculation was occuring. Fig.3.6 shows the profile matched to the CD model. The other two models did not match the data. The profile does not show any of the fast flow path characteristics. Furthermore, since the tailing section is not measured it is difficult to determine whether the recirculation effects 


\begin{tabular}{|c|c|c|c|}
\hline Model & IC \& BC & Parameters & Iution \\
\hline$C D$ & $\begin{array}{c}C(x, 0)=0 \\
\lim _{x \rightarrow \infty} C(x, t)=0 \\
C(0, t)=\frac{\mathrm{m}}{\mathrm{o}} \delta(t)+C(L, T) \\
\end{array}$ & $\begin{array}{l}\alpha_{1}=\frac{1}{p_{8}}=\frac{p}{L L} \\
\alpha_{2}=\frac{1}{l_{11}}=\frac{1}{L}\end{array}$ & $C=\frac{\pi}{6} \sum_{n=0}^{\infty} \frac{(n+1)}{2 \sqrt{\pi_{1} \alpha_{1} t^{1}}} \exp ^{-\left(1-(n+1) \alpha_{2} t\right)^{2} /\left(\alpha_{1} \alpha_{2} t\right)}:$ \\
\hline$A D$ & $\begin{array}{c}C(x, 0)=C_{m}(x, y, 0)=0 \\
\lim _{r \rightarrow \infty} C(x, t)=0 \\
C(0, t)=\frac{m}{Q} \delta(t)+C(L, t) \\
C(x, t)=C_{m}(x, 0, t) \\
\lim _{y \rightarrow \infty} C_{m}(x, y, t)=0\end{array}$ & $\begin{array}{c}\alpha_{1}=\frac{1}{t_{1}}=\frac{R}{u L} \\
\alpha_{2}=\frac{1}{t_{1}}=\frac{t}{t} \\
\alpha_{3}=\frac{1}{V_{2}}=\frac{d \sqrt{D_{2}}}{b}\end{array}$ & $C=\frac{m}{\varphi}\left(\exp ^{-1 /\left(2 \sigma_{1}\right)} \exp ^{\sqrt{1 /\left(4 \alpha_{1}^{3}\right)+\left(0+2 \sigma_{3} \sqrt{\pi}\right) /\left(m_{1} m_{2}\right)}}-1\right)^{-1}$ \\
\hline
\end{tabular}

Table 3.2: Solutions to Models of Recirculating Flow 
appeared or not. The profile could also be matched to the CD model without recirculation. Given the very long breakthrough time, and since the shape of the profile is very similar to the profile in the paper by Webster et. al. (1964), it is reasonable to use the uniform porous medium approach. If we divide the flow field into crescents such that their flow rates are equal then we can use a single transfer function for multiple paths in the regression procedure. If the flow rates were to be unequal then we would bave to use as many transfer functions as the number of paths, which is not feasible for more than a few paths. If the flow velocities of the individual paths are specified explicitly, then the number of nonlinear parameters can be reduced significantly. Further assumptions on the form of the dispersivity (for instance it can be taken as proportional to the velocity or to the square of the velocity) will greatly simplify the problem. The best approach is to start from the simplest case and to add more complications as the problem requires. The fracture aperture estimated from the match values of the CD model did not satisfy Eq.3.8. This was expected since the shape of the profile was not well captured by the model. This example however is not a characteristic profile one would expect to obtain from a single fracture flow. Therefore, it would be more appropriate to test the model on data from a longer test including strong recirculation effects.

\subsection{CONCLUSIONS}

The performances of three mathematical models to match the field data were compared. We were able to successfully match the tracer return profiles from the tests without recirculation to all three models. Therefore, to choose the appropriate model we have to consider two factors. First, we should look for a small residual error in the fit. Then we should look at how successful is the model in capturing characteristics of the return profiles, such as the breakthrough and peak arrival times. We should also evaluate whether the estimated values of the parameters such as fracture aperture are realistic or not. Based on the analysis of the return profiles of the tests from Wairakei field, New Zealand, we have arrived at the following conclusions: 
1. The CD model estimates low Peclet number values, large fracture aperture values and low flow speed values. However, it usually could not capture the distinctive features of the profiles.

2. The MD model estimates fast flow speed values and captures the long tailing response better than the CD model. However, it usually could not match the breakthrough and peak arrival times. It gives small estimates of fracture aperture.

3. The AD model matched the profiles excellently and it allowed comparison of the relative effects of the Peclet number and $\frac{1}{\sqrt{t_{d}}}$ (which account for the spreading of the tracer along the transport region).

- In the test with recirculation, however, we were able to match the return profile only with the solution to the CD model. We have suggested that a uniform porous medium approach be used in order to analyze this specific data set. Our method is still applicable and our solutions are valid except that multiple path transfer functions should be used in the regression procedure. Finally, we may conclude that the return profiles of tracer tests with recirculation provide more information, which enables us to choose the appropriate model more easily. 


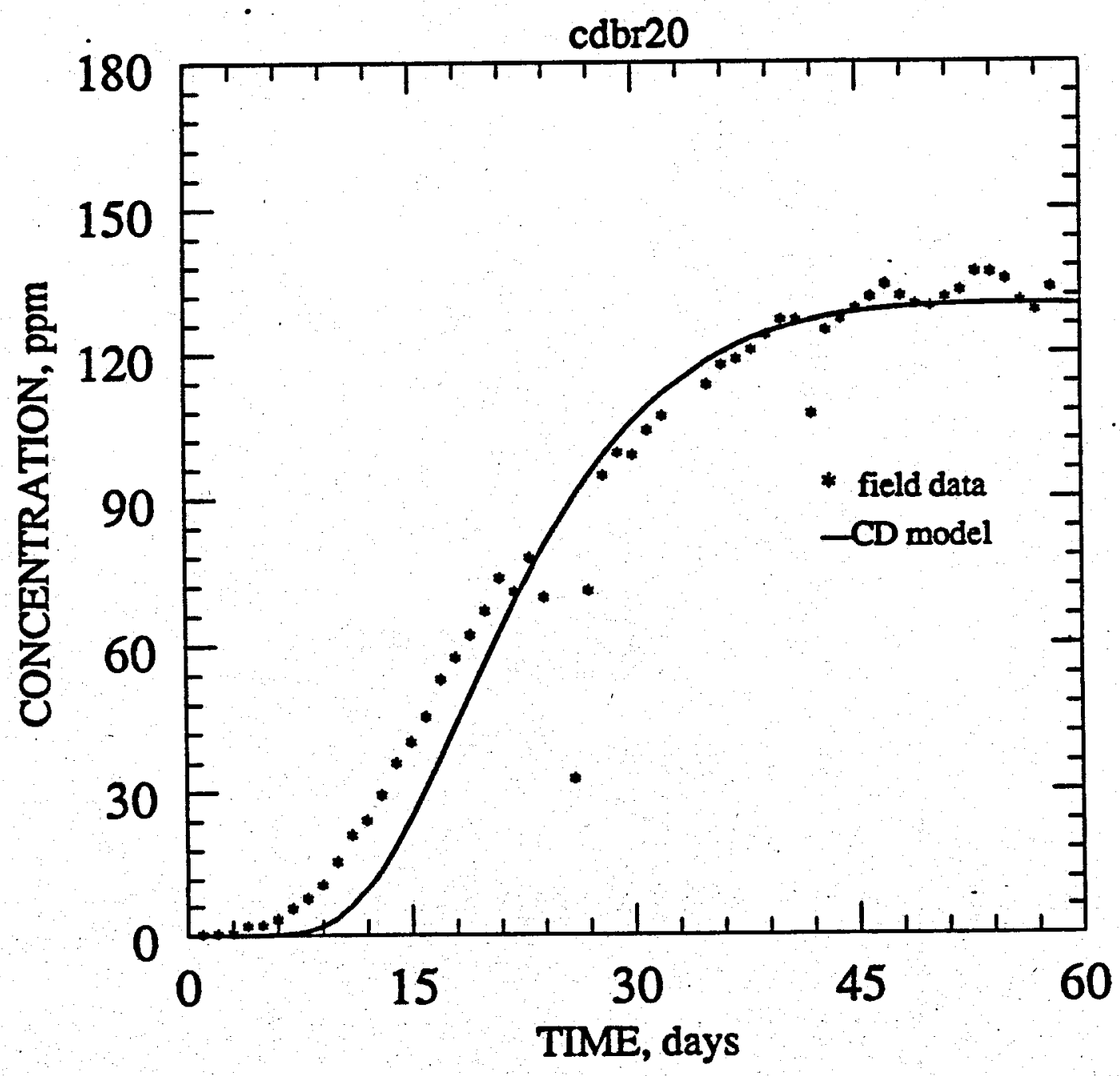

Figure 3.6: The Return Profile at BR20 Matched by the CD Model 


\section{Bibliography}

[1] Webster, D. S., Proctor, J. F. and Marine, I. W.:"Two-Well Tracer Test in Fractured Crystalline Rock," U.S. Geol. Surv. Water Supply Pap., 1544-I, 1964, 215-230

[2] Grove, D. B. and Beetem, W. A.: "Porosity and Dispersion Constant Calculations for a Fractured Carbonate Aquifer Using the Two Well Tracer Method," Water Resour. Res. 7, 1971, 128

[3] Bear, J.: Dynamics of Fluids in Porous Media, Elsevier, New York, 1972

[4] Clark, R. T.: "A Review of Some Mathematical Models Used in Hydrology with Observations on Their Calibration and Use," J. Hydr. $19,1973,1-20$

[5] Brigham, W. E.: "Mixing Equations in Short Laboratory Cores," Soc. Pet. Eng. J. 14, $1974,91-99$

[6] Kreft, A. and Zuber, A.: "On the Physical Meaning of the Dispersion Equation and its Solutions for Different Initial and Boundary Conditions," Chem. Eng. Sci. 33, $1978,1471-1480$

[7] Kreft, A. and Zuber, A.: "On the Use of the Dispersion Model of Fluid Flow," Int. J. Appl. Rad. Isot. 30, 1979 ,705-708

[8] Horne, R. N. and Rodriguez, J.: "Dispersion in Tracer Flow in Fractured Geothermal Systems," Proceedings, 7th Annual Stanford University Geothermal Workshop 1981

[9] Horne, R. N.: "Geothermal Reinjection Experience in Japan," J. Petroleum Technology, 34, 1982 495-503 
[10] Zuber, A.: "Models for Tracer Flow," In: Tracer Methods in Isotope Hydrology. Int. At. Energy Agency (I.A.E.A), Report TECDOD-291 Vienna, 1983, 67-112

[11] Parker, J. C. and van Genuchten M. Th.: "Flux-A veraged and VolumeAveraged Concentrations in Continuum Approaches to Solute Transport," Water Resour. Res. 20 7, 1984, 866-872 


\section{Chapter 4}

\section{Pressure Transient Modeling of Non-Uniformly Fractures Reservoirs}

Ashok Belani and Younes Jalali-Yazdi

\subsection{OVERVIEW}

Double porosity formulations of naturally fractured reservoirs assume fracturing is uniform and matrix block size is constant. A model is formulated that eliminates this assumption. A distribution of matrix block size is incorporated in a source integral to represent interporosity flow in a nonuniformly fractured reservoir. Uniform fracturing is a special case where the block size distribution is a shifted Dirac delta function. Block size variability affects the pressure response markedly.

\subsection{INTRODUCTION}

It is desirable to estimate the matrix block size distribution from well test data. The mean of the distribution is a measure of fracture intensity. The variance is a measure of the degree of uniformity of fracturing. Such information is necessary in many reservoir engineering calculations such as: (a) estimating recoverable heat from geothermal reservoirs, (b) estimating 
the rate of imbibition, (c) history matching reservoir performance, and (d) planning development well locations.

A model is formulated that predicts the wellbore pressure response for a fractured reservoir of varying matrix block size. This model can be used to estimate matrix block size distribution from well test data.

\subsection{THEORY}

Fluid flow in a fractured reservoir with a random distribution of matrix blocks of varying size is described by:

$$
\frac{k_{f}}{\mu} \nabla^{2} P_{f}=\phi_{f} c_{f} \frac{\partial P_{f}}{\partial t}+\int_{h_{\min }}^{h_{\max }} Q(h) f(h) d h .
$$

The source integral in Eqn. 4.1 represents matrix to fracture interporosity flow. $Q(h)$ is the flow contribution from a matrix of block size $h$, given by:

$$
Q(h)=\phi_{m} c_{m} \frac{\partial p_{m}}{\partial t}(h)
$$

for pseudo-steady state, and

$$
Q(h)=-\frac{k_{m}}{\mu h}\left|\nabla p_{m}\right|,
$$

for unsteady state interporosity flow. $\mathrm{f}(\mathrm{h})$ is the matrix block size distribution; it gives the probability of occurrence of a matrix block of size h. For a uniformly fractured reservoir, matrix block size is constant (size H) and hence:

$$
\begin{aligned}
f(h)=\delta(h-H) & =0, h \neq H, \\
& =\infty, h=H .
\end{aligned}
$$

Eqns. 4.1 and 4.4 yield the double porosity formulation of a uniformly fractured reservoir.

For a nonuniformly fractured reservoir block size varies. An intensely fractured reservoir exhibits a positively skewed matrix block size distribution, i.e., "favors" small blocks. A sparsely fractured reservoir exhibits a negatively skewed matrix block size distribution, i.e., "favors" large blocks. 
Fracturing on two scales would exhibit a bimodal block size distribution, i.e., two controlling sets of matrix blocks exist.

A block size distribution of interest is the rectangular (or uniform) distribution where all block sizes $\left(h_{\min }\right.$ to $h_{\max }$ ) are equally probable:

$$
f(h)=\frac{1}{h_{\max }-h_{\min }},
$$

with mean:

$$
h_{\text {mean }}=\frac{h_{\text {min }}+h_{\text {mex }}}{2}
$$

and variance:

$$
\sigma^{2}=\frac{1}{12}\left(h_{\max }-h_{\min }\right)^{2} .
$$

The application of the rectangular distribution is two-fold:

1. It should be used when the matrix block size distribution is unknown.

2. A sequence of rectangular distributions can be used to approximate any matrix block size distribution.

\subsection{SOLUTION}

For the radial flow of a slightly compressible fluid in a fractured reservoir of infinite extent where the pressure in the fracture network and the matrix blocks are initially at equilibrium and subsequently flow is linear in the matrix blocks and the mode of interporosity flow is pseudo-steady state, we obtain the dimensionless wellbore pressure response in Laplace space for a rectangular matrix block size distribution: ${ }^{1}$

$$
\bar{P}_{D W}=\frac{1}{s} K_{0}(\sqrt{s g(s)}),
$$

where,

$$
\begin{array}{r}
g(s)=\omega_{f}+\omega_{m} \frac{\sqrt{3 \lambda_{\min } / \omega_{m} s}}{1-\sqrt{\lambda_{\min } / \lambda_{\max }}} \times \\
\\
\left(\tan ^{-1} \sqrt{3 \lambda_{\max } / \omega_{m} s}-\tan ^{-1} \sqrt{3 \lambda_{\min } / \omega_{m} s}\right)
\end{array}
$$

\footnotetext{
${ }^{1}$ See Reference 2 for details.
} 
Parameter $\mathrm{s}$ is the Laplace variable related to dimensionless time:

$$
T_{D}=\frac{k_{f} t}{\left(\phi_{f} c_{f}+\phi_{m} c_{m}\right) \mu r_{w}^{2}}
$$

Parameters $\omega_{f}$ and $\omega_{m}$ are the dimensionless fracture and matrix storativity:

$$
\begin{gathered}
\omega_{f}=\frac{\phi_{f} c_{f}}{\phi_{f} c_{f}+\phi_{m} c_{m}}, \\
\omega_{m}=\frac{\phi_{m} c_{m}}{\phi_{f} c_{f}+\phi_{m} c_{m}}, \\
\omega_{f}+\omega_{m}=1 .
\end{gathered}
$$

Unlike the single block size formulation, Eqn. 4.9 includes two interporosity flow coefficients $\lambda_{\max }$ and $\lambda_{\min }$ which arise because matrix block size varies uniformly from $h_{\min }$ to $h_{\text {max }}$ :

$$
\begin{aligned}
& \lambda_{\min }=\frac{k_{m} r_{w}^{2}}{k_{f} h_{\max }^{2}}, \\
& \lambda_{\max }=\frac{k_{m} r_{w}^{2}}{k_{f} h_{\min }^{2}} .
\end{aligned}
$$

In general, the interporosity flow coefficient is a variable, $\lambda(h)$, specified by the matrix block size distribution.

In the limit where $\lambda_{\min }$ approaches $\lambda_{\max }$ (or vice versa), Eqn. 4.9 behaves similar to the single block size formulation of Warren and Root (Reference 1):

$$
g(s)=\omega_{f}+\omega_{m} \frac{3 \lambda}{3 \lambda+\omega_{m} s}
$$

That is, as the rectangular distribution narrows and approaches a Dirac delta function, Eqn. 4.9 reverts to the conventional uniformly fractured reservoir models. This is indicated in Figure 4.1. The derivative curve at the far right is the single block size response with $\lambda=10^{-9}$. Other derivative curves represent the uniform matrix block size distribution response with $\lambda_{\min }=10^{-9}$ and $\lambda_{\max }$ of $10^{-4}, 10^{-5}, 10^{-6}, 10^{-7}$, and $10^{-8}$. As the spread in matrix block size (or $\lambda$ ) increases, the pressure response becomes more smooth and the characteristic pressure behavior of a fractured reservoir becomes less pronounced. In such conditions, it is difficult to detect two parallel semi-log straight lines on a drawdown or a buildup plot. 


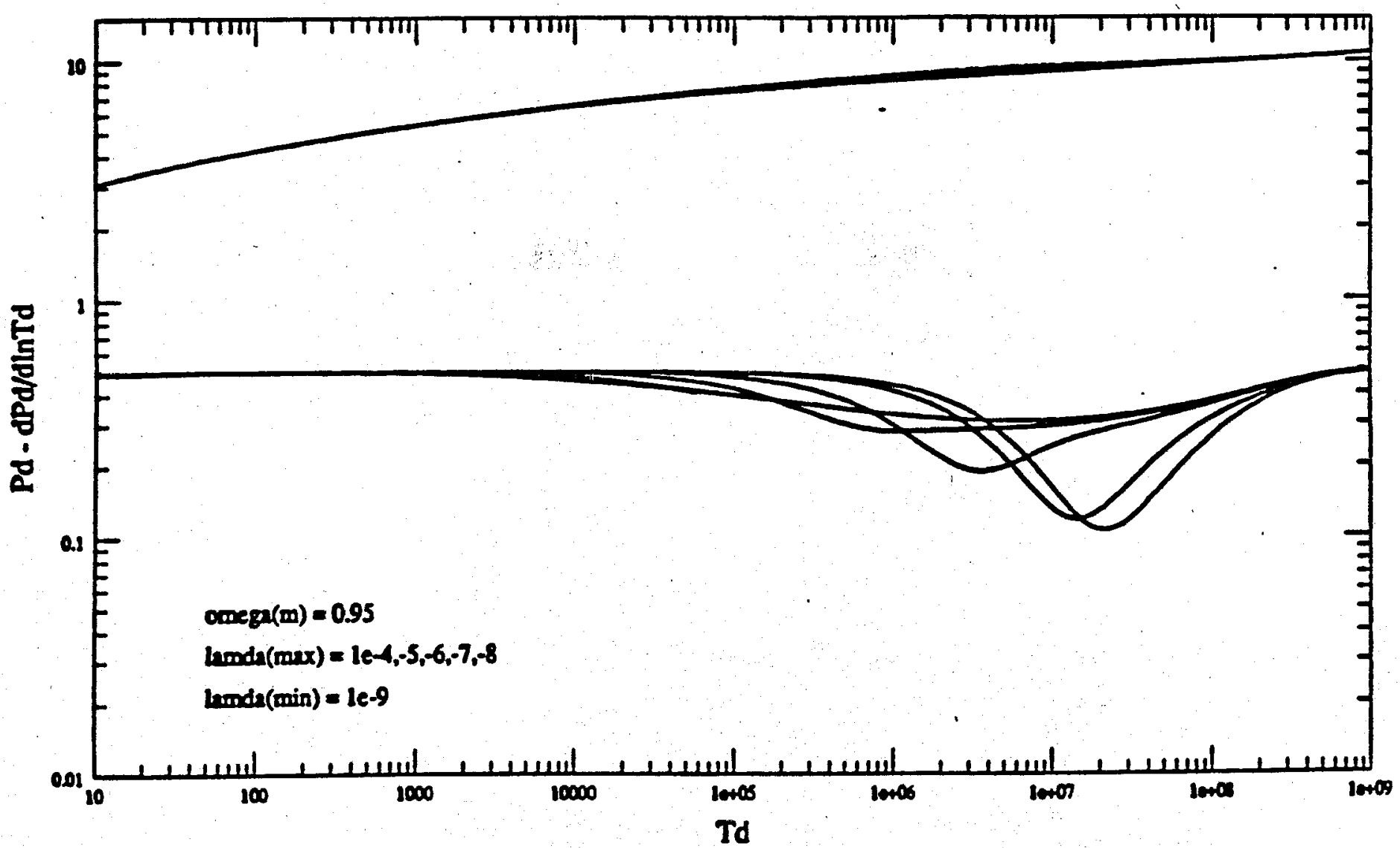

Figure 4.1: Uniform matrix block size distribution response compared to single block size response

\subsection{CONCLUSIONS}

1. A robust formulation of pressure transient response in a nonuniformly fractured reservoir is developed.

2. The characteristic pressure behavior of a naturally fractured reservoir becomes less pronounced as the variance of the matrix block size distribution increases.

\subsection{REFERENCES}

1. Warren, J.E. and Root, P.J.: "The Behavior of Naturally Fractured Reservoirs," Soc. Pet. Eng. J., September 1963.

2. Belani, A. and Jalali-Yazdi, Y.: "Estimation of Matrix Block Size Distribution and Fracture Intensity from Pressure Transient Data," paper SPE 18171, to be presented at the 63rd Annual Technical Con- 
ference \& Exhibition of the Society of Petroleum Engineers, Houston, Texas, October 2-5, 1988. 